\title{
Approximation Algorithms and Hardness of the $k$-Route Cut Problem
}

\author{
JULIA CHUZHOY and YURY MAKARYCHEV, Toyota Technological Institute at Chicago \\ ARAVINDAN VIJAYARAGHAVAN, Princeton University \\ YUAN ZHOU, Carnegie Mellon University
}

We study the $k$-route cut problem: given an undirected edge-weighted graph $G=(V, E)$, a collection $\left\{\left(s_{1}, t_{1}\right),\left(s_{2}, t_{2}\right), \ldots,\left(s_{r}, t_{r}\right)\right\}$ of source-sink pairs, and an integer connectivity requirement $k$, the goal is to find a minimum-weight subset $E^{\prime}$ of edges to remove, such that the connectivity of every pair $\left(s_{i}, t_{i}\right)$ falls below $k$. Specifically, in the edge-connectivity version, EC-kRC, the requirement is that there are at most $(k-1)$ edge-disjoint paths connecting $s_{i}$ to $t_{i}$ in $G \backslash E^{\prime}$, while in the vertex-connectivity version, VC-kRC, the same requirement is for vertex-disjoint paths. Prior to our work, poly-logarithmic approximation algorithms have been known for the special case where $k \leq 3$, but no non-trivial approximation algorithms were known for any value $k>3$, except in the single-source setting. We show an $O\left(k \log ^{3 / 2} r\right)$-approximation algorithm for EC-kRC with uniform edge weights, and several polylogarithmic bi-criteria approximation algorithms for EC-kRC and VC-kRC, where the connectivity requirement $k$ is violated by a constant factor. We complement these upper bounds by proving that VC-kRC is hard to approximate to within a factor of $k^{\epsilon}$ for some fixed $\epsilon>0$. We then turn to study a simpler version of VC-kRC, where only one source-sink pair is present. We give a simple bi-criteria approximation algorithm for this case, and show evidence that even this restricted version of the problem may be hard to approximate. For example, we prove that the single source-sink pair version of VC-kRC has no constant-factor approximation, assuming Feige's Random $\kappa$-AND assumption.

Categories and Subject Descriptors: F.2 [Theory of Computation]: Analysis of Algorithms

General Terms: Algorithms, Theory

Additional Key Words and Phrases: Approximation algorithm, $k$-route cut problem

ACM Reference Format:

Julia Chuzhoy, Yury Makarychev, Aravindan Vijayaraghavan, and Yuan Zhou. 2015. Approximation algorithms and hardness of the $k$-route cut problem. ACM Trans. Algorithms 12, 1, Article 2 (December 2015), 40 pages.

DOI: http://dx.doi.org/10.1145/2644814

\section{INTRODUCTION}

Multicommodity flows and cuts in graphs are among the most extensively studied combinatorial objects. Due to their rich connections to many combinatorial optimization problems, algorithms for various versions of flow and cut problems provide a powerful and a widely used algorithmic toolkit. One of the central problems in this area is minimum multicut: given an $n$-vertex graph $G=(V, E)$ with nonnegative weights $w_{e}$ on edges $e \in E$ and a collection $\left\{\left(s_{1}, t_{1}\right),\left(s_{2}, t_{2}\right), \ldots,\left(s_{r}, t_{r}\right)\right\}$ of source-sink pairs, find a

This work was supported in part by NSF CAREER award CCF-0844872 and the Sloan Research Fellowship. Authors' addresses: J. Chuzhoy and Y. Makarychev, Toyota Technological Institute at Chicago, 6045 S. Kenwood Ave., Chicago, IL 60637; emails: \{cjulia, yury\}@ttic.edu; A. Vijayaraghavan, EECS, Northwestern University, 2145 Sheridan Rd, Tech L359, Evanston, IL 60208; email: aravindv@eecs.northwestern.edu; Y. Zhou, Mathematics Department, Massachusetts Institute of Technology, 77 Massachusetts Ave, Cambridge, MA 02139; email: yuanzhou@mit.edu.

Permission to make digital or hard copies of part or all of this work for personal or classroom use is granted without fee provided that copies are not made or distributed for profit or commercial advantage and that copies show this notice on the first page or initial screen of a display along with the full citation. Copyrights for components of this work owned by others than ACM must be honored. Abstracting with credit is permitted. To copy otherwise, to republish, to post on servers, to redistribute to lists, or to use any component of this work in other works requires prior specific permission and/or a fee. Permissions may be requested from Publications Dept., ACM, Inc., 2 Penn Plaza, Suite 701, New York, NY 10121-0701 USA, fax +1 (212) 869-0481, or permissions@acm.org.

(c) 2015 ACM 1549-6325/2015/12-ART2 $\$ 15.00$

DOI: http://dx.doi.org/10.1145/2644814 
minimum-weight subset $E^{\prime}$ of edges to delete so that each pair $\left(s_{i}, t_{i}\right)$ is disconnected in the resulting graph $G \backslash E^{\prime}$. The dual to minimum multicut is the maximum multicommodity flow problem, where the goal is to find a maximum flow between the pairs $\left(s_{i}, t_{i}\right)$, with the restriction that each edge $e$ carries at most $w_{e}$ flow units. It is easy to see that minimum multicut can be viewed as revealing a bottleneck in the routing capacity of $G$, as the value of any multicommodity flow cannot exceed the value of the minimum multicut in G. A fundamental result, due to Leighton and Rao [1999] and Garg et al. [1995] shows that the value of minimum multicut is within an $O(\log r)$ factor of that of maximum multicommodity flow in any graph, where $r$ is the number of the source-sink pairs. This result can be seen as an extension of the famous min-cut max-flow theorem to the multicommodity setting, and it also gives an efficient $O(\log r)$-approximation algorithm for minimum multicut — the best currently known approximation guarantee for the problem.

In this article, we study a natural generalization of minimum multicut-the minimum $k$-route cut problem. In this problem, the input again consists of an $n$-vertex graph $G=(V, E)$ with nonnegative weights $w_{e}$ on edges $e \in E$, and a collection $\left\{\left(s_{1}, t_{1}\right),\left(s_{2}, t_{2}\right), \ldots,\left(s_{r}, t_{r}\right)\right\}$ of $r$ source-sink pairs. Additionally, we are given an integral connectivity threshold $k>0$. The goal is to find a minimum-weight subset $E^{\prime} \subseteq E$ of edges to delete, such that the connectivity of each pair $\left(s_{i}, t_{i}\right)$ falls below $k$ in the resulting graph $G \backslash E^{\prime}$. We study two versions of this problem: in the edge-connectivity version (EC-kRC), the requirement is that for each $1 \leq i \leq r$, the number of edge-disjoint paths connecting $s_{i}$ to $t_{i}$ in graph $G \backslash E^{\prime}$ is less than $\bar{k}$. In the vertex-connectivity version (VC-kRC), the requirement is that the number of vertex-disjoint paths connecting $s_{i}$ to $t_{i}$ is less than $k$. It is not hard to see that VC-kRC captures EC-kRC as a special case (see Appendix A) and hence is more general. It is also easy to see that minimum multicut is a special case of both EC-kRC and VC-kRC, with the connectivity requirement $k=1$. We also consider a special case of EC-kRC, where all edges have unit weight, and we refer to it as the uniform EC-kRC. We note that for VC-kRC, the uniform and the nonuniform edge-weight versions are equivalent up to a small loss in the approximation factor, as shown in Appendix B, and so we do not distinguish between them.

The primary motivation for studying $k$-route cuts comes from multicommodity flows in fault-tolerant settings, where the resilience to edge and node failures is important. An elementary $k$-route flow between a pair $s$ and $t$ of vertices is a set of $k$ disjoint paths connecting $s$ to $t$. A $k$-route (st)-flow is just a combination of such elementary $k$-route flows, where each elementary flow is assigned some fractional value. This is a natural generalization of the standard (st)-flows, which ensures that the flow is resilient to the failure of up to $(k-1)$ edges or vertices. Multiroute flows were first introduced by Kishimoto [1996] and have since been studied in the context of communication networks [Bagchi et al. 2003, 2007; Aneja et al. 2007]. In a series of papers, Kishimoto [1996], Kishimoto and Takeuchi [1993], and Aggarwal and Orlin [2002] have developed a number of efficient algorithms for computing maximum multiroute flows. As in the case of standard flows, we can extend $k$-route (st)-flows to the multicommodity setting, where the goal is to maximize the total $k$-route flow between all source-destination pairs. It is easy to see that the minimum $k$-route cut is a natural upper bound on the maximum $k$-route flow-just like minimum multicut upper bounds the value of the maximum multicommodity flow. Hence, as in the case with the standard multicut, multiroute cuts can be seen as revealing the network bottleneck, and so the minimum $k$-route cut in a graph captures the robustness of real-life computer and transportation networks.

The first approximation algorithm for the EC-kRC problem, due to Chekuri and Khanna [2008], achieved a factor $O\left(\log ^{2} n \log r\right)$-approximation for the special case where $k=2$, by rounding a linear programming relaxation. This was improved by 
Table I. Upper Bounds for EC-kRC

\begin{tabular}{|l|l|l|}
\hline & \multicolumn{1}{|c|}{ Previous Results } & \multicolumn{1}{c|}{ Current Work } \\
\hline$k=2$ & $\begin{array}{l}O\left(\log ^{2} r\right)[\text { Barman and } \\
\text { Chawla 2010] }\end{array}$ & $O\left(\log ^{1.5} r\right)$ \\
\hline $\begin{array}{l}O\left(\log ^{3} r\right)[\text { Kolman and } \\
\text { Scheideler 2011] }\end{array}$ & - & $\begin{array}{l}O\left(k \log ^{1.5} r\right),\left(1+\delta, O\left(\frac{1}{\delta} \log ^{1.5} r\right)\right) \text { for any } \\
\operatorname{constant~} 0<\delta<1\end{array}$ \\
\hline arbitrary $k$, uniform & - & $\begin{array}{l}(2, O(\log 2.5 \\
(O \log \log r)) \text { in time } n^{O(k)} ;\end{array}$ \\
\hline arbitrary $k$, general & - & $\left.\left(\log ^{3} r\right)\right)$ in poly $(n)$-time \\
\hline
\end{tabular}

Note: Running time is polynomial in $n$ and $k$ unless stated otherwise.

Barman and Chawla [2010] to give an $O\left(\log ^{2} r\right)$-approximation algorithm for the same version, by generalizing the region-growing LP-rounding scheme [Leighton and Rao 1999; Garg et al. 1995]. They note that it seems unlikely that their algorithm can be extended to handle higher values of $k$ using similar techniques. Very recently, Kolman and Scheideler [2011] obtained a $O\left(\log ^{3} r\right)$ approximation to EC-3RC ( $k=3$ case) from the linear program of Barman and Chawla [2010] by using a multilevel ball growing rounding. To the best of our knowledge, no approximation algorithms with subpolynomial (in $n$ ) guarantees are known for any variant of the problem, for any value $k>3$, except in the single-source setting that we discuss later. Our first result is an $O\left(k \log ^{1.5} r\right)$-approximation algorithm for the uniform version of EC-kRC.

Since the problem appears to be computationally difficult, it is natural to turn to bi-criteria approximation by slightly relaxing the connectivity requirement. Given parameters $\alpha, \beta>1$, we say that an algorithm is an $(\alpha, \beta)$-bi-criteria approximation for EC-kRC (or VC-kRC) if it is guaranteed to produce a valid $k^{\prime}$-route cut of weight at most $\beta$. OPT, where $k^{\prime} \leq \alpha k$, and OPT is the value of the optimal $k$-route cut. Indeed, we can do much better in the bi-criteria setting: we obtain a $\left(1+\delta, O\left(\frac{1}{\delta} \log ^{1.5} r\right)\right)$-bicriteria approximation for any constant $0<\delta<1$ for the uniform EC-kRC problem (notice that the factors do not depend on $k$ ). When edge weights are arbitrary, we obtain a $\left(2, \tilde{O}\left(\log ^{2.5} r\right)\right)$-bi-criteria approximation in $n^{O(k)}$ time and an $\left(O(\log r), O\left(\log ^{3} r\right)\right)$-bicriteria approximation in time polynomial in $n$ and $k$. We also show an $O\left(\log ^{1.5} r\right)$ approximation for the special case where $k=2$, thus slightly improving the result of Barman and Chawla [2010]. The previously known upper bounds and our results for EC-kRC are summarized in Table I.

We note that on the inapproximability side, it is easy to show that for any value of $k, \mathrm{EC}-\mathrm{kRC}$ is at least as hard as minimum multicut, up to small constant factors. ${ }^{1}$ Although minimum multicut is known to be hard to approximate up to any constant factor assuming the unique games conjecture [Khot and Vishnoi 2005; Chawla et al. 2006], it is only known to be NP-hard to approximate to within a small constant factor [Dahlhaus et al. 1994]. In fact, one of the motivations for studying $k$-route cuts is that inapproximability results may yield insights into approximation hardness of multicut.

We now turn to the more general VC-kRC problem. The $O\left(\log ^{2} n \log r\right)$-approximation of Chekuri and Khanna [2008], and the $O\left(\log ^{2} r\right)$-approximation of Barman and Chawla [2010] for 2-route cuts extend to the vertex-connectivity version as well, as does our $O\left(\log ^{1.5} r\right)$-approximation algorithm. Prior to our work, no nontrivial approximation algorithms were known for any higher values of $k$. In this article, we show a

\footnotetext{
$\overline{{ }^{1} \mathrm{~A} \text { simple }}$ reduction replaces every vertex $v$ of the multicut instance by a set $S_{v}$ of $M$ vertices, where $M \gg k$, and every edge $(u, v)$ by a set of $M^{2}$ edges connecting every vertex of $S_{v}$ to every vertex of $S_{u}$.
} 
Table II. Results for VC-kRC

\begin{tabular}{|l|l|l|}
\hline & \multicolumn{1}{|c|}{ Previous Results } & \multicolumn{1}{c|}{ Current Work } \\
\hline$k=2$ & $O\left(\log ^{2} r\right)$ [Barman and Chawla 2010] & $O\left(\log ^{1.5} r\right)$ \\
\hline arbitrary $k$ & $\begin{array}{l}\text { APX-hard [Dahlhaus et al. 1994] } \\
\text { no constant factor approximation under } \\
\text { UGC [Khot and Vishnoi 2005; Chawla } \\
\text { et al. 2006] }\end{array}$ & $\begin{array}{l}\left(2, O\left(d k \log ^{2.5} r \log \log r\right)\right) \text {-approximation } \\
\text { algorithm, running time } n^{O(k)}, \text { where } d \text { is } \\
\text { the maximum number of demand pairs in } \\
\text { which any terminal participates } \\
\Omega\left(k^{\epsilon}\right) \text {-hardness for some constant } \epsilon>0\end{array}$ \\
\hline
\end{tabular}

Table III. Results for (st)-EC-kRC and (st)-VC-kRC

\begin{tabular}{|l|l|}
\hline \multicolumn{2}{|c|}{ Previous Results } \\
\hline$k$ is a constant & can be solved exactly \\
\hline (st)-EC-kRC arbitrary $k$ & $2(k-1)$-approximation for the uniform case [Bruhn et al. 2008] \\
& $(4,4)$-bi-criteria approximation [Barman and Chawla 2010] \\
& $(2,2)$-bi-criteria approximation for the uniform case [Barman and Chawla \\
& 2010] \\
& (st)-EC-kRC with edge capacities is NP-hard [Barman and Chawla 2010] \\
\hline \multicolumn{2}{|c|}{ Current Work } \\
\hline (st)-VC-kRC, arbitrary $k$ & $(k+1)-$ approximation \\
& $(1+1 / c, 1+c)$-bi-criteria approximation (for every constant $c)$ \\
& no $\left(1+\gamma_{C}, C\right)$-bi-criteria approximation assuming Feige's random $\kappa$-AND \\
& hypothesis (for every $C$ and sufficiently small constant $\left.\gamma_{C}\right)$ \\
& $\rho$ approximation algorithm for (st)-VC-kRC would lead to a \\
& $2 \rho^{2}$-approximation for densest $\kappa$-subgraph \\
\hline
\end{tabular}

$\left(2, \tilde{O}\left(k d \log ^{2.5} r\right)\right)$-bi-criteria approximation algorithm for VC-kRC, with running time $n^{O(k)}$, where $d$ is the maximum number of demand pairs in which any terminal participates. We note that, as in the case of EC-kRC, for any value of $k, \mathrm{VC}-\mathrm{kRC}$ is at least as hard to approximate as minimum multicut (up to small constant factors), and to the best of our knowledge, no other inapproximability results have been known for this problem. We show that VC-kRC is hard to approximate up to any factor better than $\Omega\left(k^{\epsilon}\right)$ for some constant $\epsilon>0$. Our results for VC-kRC are summarized in Table II.

To better understand the multiroute cut problem computationally, it is instructive to consider a simpler special case, where we are only given a single source-sink pair $(s, t)$. We refer to this special case of VC-kRC and EC-kRC as (st)-VC-kRC and (st)-EC-kRC, respectively. As in the general case, it is easy to see that (st)-EC-kRC can be cast as a special case of (st)-VC-kRC. When the connectivity requirement $k$ is constant, both problems can be solved efficiently as follows: guess a set $E^{\prime}$ of $(k-1)$ edges, and compute the minimum edge (st)-cut in graph $G \backslash E^{\prime}$. The algorithm for (st)-VC-kRC is similar except that we guess a set $V^{\prime}$ of $(k-1)$ vertices, and compute the minimum edge (st)-cut in graph $G \backslash V^{\prime}$. However, for larger values of $k$, only a $2(k-1)$-approximation is known for (st)-EC-kRC, for the special case where the edge weights are uniform, due to Bruhn et al [2008]. ${ }^{2}$ Barman and Chawla [2010] show that a generalization of (st)-EC-kRC where edges are allowed to have capacities is NP-hard. As no good approximation guarantees are known for the problem, it is natural to turn to bi-criteria approximation. For general values of $k$, Barman and Chawla [2010] have shown a (4,4)-bi-criteria approximation algorithm for (st)-EC-kRC, and a (2,2)-bi-criteria approximation for uniform (st)-EC-kRC. In fact, all of these algorithms extend to a single-source multiplesink scenario, except that the factor $(4,4)$-approximation requires that the number of terminals is constant. In this article, we focus on the more general node-connectivity version of the problem. We start by showing a simple factor $(k+1)$-approximation

\footnotetext{
${ }^{2}$ This result also extends to the single-source multiple-sinks setting.
} 
algorithm for (st)-VC-kRC and a factor $\left(1+\frac{1}{c}, 1+c\right)$-bi-criteria approximation for any constant $c$. We then complement these upper bounds by providing evidence that the problem is hard to approximate. Specifically, we show that for any constant $C$, there is no $(1+\gamma, C)$-bi-criteria approximation for (st)-VC-kRC, assuming Feige's random $\kappa$-AND hypothesis [Feige 2002], where $\gamma$ is some small constant depending on $C$. We also show that a factor $\rho$ approximation algorithm for (st)-VC-kRC would lead to a factor $2 \rho^{2}$-approximation for the densest $\kappa$-subgraph problem. These inapproximability results are inspired by the recent work of Alon et al. [2011], who have ruled out a constant factor approximation for densest $\kappa$-subgraph assuming Feige's random $\kappa$-AND hypothesis.

Recall that the densest $\kappa$-subgraph problem takes as input a graph $G=(V, E)$ on $n$ vertices and a parameter $\kappa$, and asks for a subgraph of $G$ on at most $\kappa$ vertices containing the maximum number of edges. Although it is a fundamental graph optimization problem, there is a huge gap between the best known approximation algorithms and the known inapproximability results. The current best approximation algorithm, due to Bhaskara et al. [2010], achieves an $O\left(n^{1 / 4+\epsilon}\right)$-approximation in time $n^{O(1 / \epsilon)}$ for any constant $\epsilon>0$. On the negative side, Feige [2002] showed a small constant factor inapproximability using the random 3-SAT assumption, and later Khot [2004] used quasirandom PCPs to rule out a PTAS, assuming NP $\nsubseteq \bigcap_{\epsilon>0} \operatorname{BPTIME}\left(2^{n^{\epsilon}}\right)$. Raghavendra and Steurer [2010] and Alon et al. [2011] ruled out constant factor approximation algorithms for densest $\kappa$-subgraph under other less standard complexity assumptions. The densest $\kappa$-subgraph problem can also be generalized to $\lambda$-uniform hypergraphs, where the goal is again to find a subset of $\kappa$ vertices containing maximum possible number of hyperedges. We show that for any constant $\lambda \geq 2$, a factor $\rho$ approximation algorithm for (st)-VC-kRC would lead to a factor $\left(2 \rho^{\lambda}\right)$-approximation for the $\lambda$-uniform densest $\kappa$-subgraph. We note that Applebaum [2011] has shown that for $\lambda \geq 3$, the $\lambda$-uniform densest $\kappa$-subgraph problem is hard to approximate to within $n^{\epsilon}$-factor for some constant $\epsilon>0$ assuming the existence of a certain family of one-way functions.

All of our inapproximability results for (st)-VC-kRC are proved using a "proxy" problem: small set vertex expansion (SSVE). In this problem, we are given a bipartite graph $G=(U, V, E)$ and a parameter $0 \leq \alpha \leq 1$. The goal is to find a subset $S \subseteq U$ of $\alpha \cdot|U|$ vertices while minimizing the number of its neighbors $|\Gamma(S)|$. We show an approximation-preserving reduction from SSVE to (st)-VC-kRC and then prove inapproximability results for the SSVE problem. In particular, we show that approximating SSVE is almost as hard as approximating the densest $\kappa$-subgraph problem (i.e., if there is a $\rho$ approximation algorithm for SSVE, then there is a $\left(2 \rho^{2}\right)$-approximation algorithm for the densest $\kappa$-subgraph problem). This result suggests that although the SSVE problem looks similar to the small set expansion (SSE) problem [Raghavendra and Steurer 2010], it might be much harder than SSE. On the other hand, the SSVE problem is of independent interest-besides its application to the (st)-VC-kRC problem, Applebaum et al. [2010] used a "planted" version of SSVE as a hardness assumption to construct a public key encryption scheme.

Other related work. Another version of the EC-kRC problem that has received a significant amount of attention recently is the single-source setting. In this setting, we are given a single source $s$ and a set $T$ of $r$ terminals. The source-sink pairs are then set to be $\{(s, t)\}_{t \in T}$. Bruhn et al. [2008] have shown a factor 2(k-1)-approximation for the uniform version of this problem, and Barman and Chawla [2010] have shown a factor $(6, O(\sqrt{r} \ln r))$-bi-criteria approximation for the general version, a factor $(4,4)$ bi-criteria approximation for the general version where $r$ is a constant, and a factor $(2,4)$-approximation for the uniform version and arbitrary $r$. 
The (st)-EC-kRC and (st)-VC-kRC problems capture two natural budgeted cut minimization problems. The first is the minimum unbalanced cut problem [Hayrapetyan et al. 2005], in which we are given a graph $G$ with a source vertex $s$ and a budget $B$. The goal is to find a cut $(S, \bar{S})$ in $G$ with $s \in S$ and $|E(S, \bar{S})| \leq B$ while minimizing $|S|$. Hayrapetyan et al. [2005] obtain a $(1+1 / \lambda, \lambda)$-bi-criteria approximation algorithm for any $\lambda>1$ by rounding a Lagrangean relaxation for the problem. Given an instance $G=(V, E)$ of the minimum unbalanced cut problem, we can transform it into an instance of (st)-EC-kRC by setting the weights of all edges in $E$ to $\infty$, adding a sink $t$ that connects to every vertex in $V$ with a unit-weight edge, and setting $k=B$. The other problem is the minimum $k$-size (st)-cut problem, where we are given a graph $G=(V, E)$ with a special source vertex $s$ and a parameter $k$, and the goal is to find a cut $(S, \bar{S})$ in $G$ with $s \in S$ and $|S| \leq k$, minimizing the size of the cut $|E(S, \bar{S})|$. Li and Zhang [2010] give an $O(\log n)$-approximation to this problem using Räcke's graph decomposition [Räcke 2008]. This problem can be reduced to (st)-EC-kRC by assigning unit weights to the edges of $E$, and adding a sink $t$ with infinity-weight edges $(v, t)$ for each $v \in V$; the parameter $k$ remains unchanged.

\section{Our Results and Techniques}

The following two theorems summarize our results for the EC-kRC problem.

THEOREM 1.1. There is an efficient $O\left(k \log ^{1.5} r\right)$-approximation algorithm and $a(1+$ $\delta, O\left(\frac{1}{\delta} \log ^{1.5} r\right)$ )-bi-criteria approximation algorithm for any constant $\delta \in(0,1)$ for the uniform $\mathrm{EC}-\mathrm{kRC}$ problem.

THEOREM 1.2. There is a $\left(2, O\left(\log ^{2.5} r \log \log r\right)\right)$-bi-criteria approximation algorithm with running time $n^{O(k)}$ and an $\left(O(\log r), O\left(\log ^{3} r\right)\right)$-bi-criteria approximation algorithm with running time $\operatorname{poly}(n)$ for the $\mathrm{EC}-\mathrm{kRC}$ problem.

We now proceed to discuss our techniques. Our algorithms are based on a simple iterative approach: find a "sparse" cut that separates some demand pairs, remove all cut edges except for the $(k-1)$ most expensive ones from the graph, also remove all demand pairs that are no longer $k$-connected, and then recursively solve the resulting instance. The main challenge in this approach is to ensure that the cost of the removed edges is bounded by the cost of the optimal solution. To achieve this, we use a modified notion of sparsity-we use the k-route sparsity of a cut, which is the cost of all but $(k-1)$ most expensive edges of the cut divided by the number of separated terminals (see the following for formal definitions). This is necessary since the standard sparsest cut can be prohibitively expensive; its cost cannot be bounded in terms of the cost of the optimal solution. We prove, however, that the cost of the $k$-route sparsest cut can be bounded in terms of the cost of the optimal solution and thus obtain guarantees on the performance of our algorithms. This is the most technically challenging part of the analysis of our algorithms.

We extend our bi-criteria approximation for EC-kRC to the more general VC-kRC problem in the following theorem.

THEOREM 1.3. There is a $\left(2, O\left(d k \log ^{2.5} r \log \log r\right)\right)$-bi-criteria approximation algorithm for $\mathrm{VC}-\mathrm{kRC}$ with running time $n^{O(k)}$, where $d$ is the maximum number of demand pairs in which any terminal participates.

We also prove the following hardness of approximation result for VC-kRC, whose proof uses ideas similar to those used by Kortsarz et al. [2004] and Chakraborty et al. [2008] to prove hardness of vertex-connectivity network design in the following theorem. 
THEOREM 1.4. There are constants $0<\epsilon<1, k_{0}>1$ such that for any constant $\eta$, for any $k=O\left(2^{(\log n)^{1-\eta}}\right)$, where $k>k_{0}$, there is no $k^{\epsilon}$-approximation algorithm for $\mathrm{VC}-\mathrm{kRC}$, under the assumption that $\mathrm{P} \neq \mathrm{NP}$ for constant $k$, and under the assumption that NP $\nsubseteq \mathrm{DTIME}\left(n^{\text {poly } \log n}\right)$ for super-constant $k$.

Finally, for the special case of $k=2$, we obtain a slightly improved approximation algorithm in the following theorem.

THEOREM 1.5. There is an efficient factor $O\left(\log ^{1.5} r\right)$-approximation algorithm for both VC-kRC and EC-kRC when $k=2$.

We now turn to the single (st)-pair version of the problems. We start with a simple approximation algorithm, summarized in the next theorem.

THEOREM 1.6. There is an efficient factor $(k+1)$-approximation algorithm, and for every constant $c>0$, there is an efficient $\left(1+\frac{1}{c}, 1+c\right)$-bi-criteria approximation algorithm for both (st)-VC-kRC and (st)-EC-kRC.

We then proceed to show inapproximability results for the single (st)-pair version of the problem. Our first inapproximability result uses Feige's random $\kappa$-AND assumption [Feige 2002]. Given parameters $n$ and $\Delta$, a random $\kappa$-AND instance is defined to be a $\kappa$-AND formula on $n$ variables and $m=\Delta n$ clauses, where each clause chooses $\kappa$ literals uniformly at random from the set of $2 n$ available literals. We say that a formula $\Phi$ is $\alpha$-satisfiable if and only if there is an assignment to the variables that satisfies an $\alpha$-fraction of the clauses. Notice that a random assignment satisfies a $1 / 2^{\kappa}$-fraction of the clauses in expectation, and we expect that this is a typical number of simultaneously satisfiable clauses for a random $\kappa$-AND formula. We next state the random $\kappa$-AND conjecture of Feige [2002] and our inapproximability result for (st)-VC-kRC.

Hypothesis 1.1 (Random $\kappa$-AND Assumption: Hypothesis 3 in Feige [2002]). For some constant $c_{0}>0$, for every $\kappa$, there is a value of $\Delta_{0}$ such that for every $\Delta>\Delta_{0}$, there is no polynomial time algorithm that for random $\kappa$-AND formulas with $n$ variables and $m=\Delta$ clauses outputs 'typical' with probability $1 / 2$, but never outputs 'typical' on instances with $\mathrm{m} / 2^{c_{0} \sqrt{\kappa}}$ simultaneously satisfiable clauses.

Theorem 1.7. For every constant $C>0$, there exists a small constant $0<\gamma<1$ that depends only on $C$ such that assuming Hypothesis 1.1, there is no polynomial time algorithm that obtains a $(1+\gamma, C)$-bi-criteria approximation for the (st)-VC-kRC problem.

We also prove a slightly different inapproximability result based on the slightly weaker random 3-SAT assumption of Feige. Given parameters $n$ and $\Delta$, a random 3SAT formula on $n$ variables and $m=\Delta n$ clauses is constructed as follows. Each clause chooses three literals uniformly at random among all available literals. Notice that a random assignment satisfies a 7/8-fraction of clauses in expectation. Next is Feige's 3-SAT assumption and our inapproximability result for (st)-VC-kRC.

HyPOTHESIS 1.2 (Random 3-SAT ASSUMPTION: HyPothesis 2 FROM FEIGE [2002]). For every fixed $\epsilon>0$, for $\Delta$ a sufficiently large constant independent of $n$, there is no polynomial time algorithm that on a random 3CNF formula with $n$ variables and $m=\Delta n$ clauses outputs 'typical' with probability at least $1 / 2$, but never outputs 'typical' when the formula is $(1-\epsilon)$-satisfiable (i.e., there is an assignment satisfying simultaneously $(1-\epsilon)$ m clauses).

THEOREM 1.8. Assuming Hypothesis 1.2, for any constant $\epsilon>0$, no polynomial-time algorithm achieves a $\left(\frac{25}{24}-\epsilon, 1.1-\epsilon\right)$-bi-criteria approximation for (st)-VC-kRC. 
Finally, we show that an existence of a good approximation algorithm for (st)-VC-kRC would imply a good approximation for the $\lambda$-uniform hypergraph densest $\kappa$-subgraph problem. Recall that in the $\lambda$-uniform hypergraph densest $\kappa$-subgraph problem, we are given a graph $G=(V, E)$, where $E$ is the set of $\lambda$-uniform hyperedges, and a parameter $\kappa$. The goal is to find a subset $S \subseteq V(G)$ of $\kappa$ vertices, maximizing the number of hyperedges $e \subseteq S$. Notice that for $\lambda=2$, this is the standard densest $\kappa$-subgraph problem.

THEOREM 1.9. For any constant $\lambda \geq 2$, and for any approximation factor $\rho$ (that may depend on $n$ ), if there is an efficient factor $\rho$ approximation algorithm for the (st)-VC$\mathrm{kRC}$ problem, then there is an efficient factor $\left(2 \rho^{\lambda}\right)$-approximation algorithm for the $\lambda$-uniform hypergraph densest $\kappa$-subgraph problem.

We note that Theorem 1.9, combined with the recent result of Alon et al. [2011] immediately implies super-constant inapproximability for (st)-VC-kRC under Hypothesis 1.1. However, our proof of Theorem 1.7 is conceptually simpler and also leads to a bi-criteria inapproximability.

Organization. We present notation and definitions and prove some results that we use throughout the article in Section 2. We study the uniform case of EC-kRC in Section 3 and the nonuniform case in Section 4. We describe our results for VC-kRC in Section 5. Then we present an algorithm for 2-route cuts in Section 6 . We prove $n^{\epsilon}$ hardness of VC-kRC in Section 7. Finally, we study the single source-sink case in Section 8, where we present an approximation algorithm and prove several hardness results for the problem.

\section{PRELIMINARIES}

In all of our problems, the input is an undirected $n$-vertex graph $G=(V, E)$ with nonnegative weights $w(e)$ on edges $e \in E$ and a parameter $k$. Additionally, we are given a set $D=\left\{\left(s_{1}, t_{1}\right), \ldots,\left(s_{r}, t_{r}\right)\right\}$ of source-sink pairs that we also refer to as demand pairs. We let $T \subseteq V$ be the subset of vertices that participate in any demand pairs, and we refer to the vertices in $T$ as terminals. For every vertex $v \in V$, let $D_{v}$ be the number of demand pairs in which $v$ participates. Given a subset $S \subseteq V$ of vertices, let $D(S)=\sum_{v \in S} D_{v}$ be the total number of terminals in $S$, counting multiplicities. We also denote by $D(S, \bar{S})$ the number of demand pairs $\left(s_{i}, t_{i}\right)$ with $s_{i} \in S, t_{i} \in \bar{S}$, or $s_{i} \in \bar{S}$ and $t_{i} \in S$. Given any subset $E^{\prime} \subseteq E$ of edges, we denote by $w\left(E^{\prime}\right)=\sum_{e \in E^{\prime}} w(e)$ its weight. Given a subset $S$ of vertices, we denote the graph induced on $G$ by $S$ by $G[S]$. Throughout the article, we denote by $E^{*}$ the optimal solution to the given EC-kRC or VC-kRC problem instance, and by OPT its value.

One of the main ideas in our algorithms is to relate the value of the appropriately defined sparsest cut in graph $G$ to the value of the optimal solution to the $k$-route cut problem. We now define the different variations of the sparsest cut problem that we use.

The sparsest cut problem. Given any cut $(S, \bar{S})$ in graph $G$, its uniform sparsity is defined to be

$$
\Phi(S)=\frac{w(E(S, \bar{S}))}{\min \{D(S), D(\bar{S})\}} .
$$

The uniform sparsity $\Phi(G)$ of the graph $G$ is the minimum sparsity of any cut in $G$,

$$
\Phi(G)=\min _{\substack{S \subset V: \\ D(S), D(\overline{\bar{S}})>0}}\{\Phi(S)\} .
$$


We use the $O(\sqrt{\log r})$-approximation algorithm for the uniform sparsest cut problem due to Arora et al. [2004]. Let $\mathcal{A}_{\mathrm{ARV}}$ denote this algorithm, and let $\alpha_{\mathrm{ARV}}(r)=O(\sqrt{\log r})$ denote its approximation factor. Given an edge-weighted graph $G$ and a set $D$ of $r$ demand pairs, algorithm $\mathcal{A}_{\mathrm{ARV}}$ finds a subset $S \subseteq V$ of vertices with $\Phi(S) \leq \alpha_{\mathrm{ARV}}(r)$. $\Phi(G)$.

Given any cut $(S, \bar{S})$ in graph $G$, its nonuniform sparsity is defined to be

$$
\tilde{\Phi}(S)=\frac{w(E(S, \bar{S}))}{D(S, \bar{S})} .
$$

The nonuniform sparsity $\tilde{\Phi}(G)$ of the graph $G$ is

$$
\tilde{\Phi}(G)=\min _{\substack{S \subset V: \\ D(S, \bar{S})>0}}\{\tilde{\Phi}(S)\} .
$$

We also use the $O(\sqrt{\log r} \cdot \log \log r)$-approximation algorithm for the nonuniform sparsest cut problem of Arora et al. [2005]. Let $\mathcal{A}_{\mathrm{ALN}}$ denote this algorithm, and let $\alpha_{\mathrm{ALN}}(r)=O(\sqrt{\log r} \cdot \log \log r)$ denote its approximation factor. Given an edge-weighted graph $G$ with a set $D$ of $r$ demand pairs, algorithm $\mathcal{A}_{\mathrm{ALN}}$ finds a subset $S \subseteq V$ of vertices with $\tilde{\Phi}(S) \leq \alpha_{\mathrm{ALN}}(r) \cdot \tilde{\Phi}(G)$.

We next generalize the notion of the sparsest cut to the multiroute setting. Given a subset $S \subseteq V$ of vertices, let $F$ denote the set of $(k-1)$ most expensive edges of $E(S, \bar{S})$, breaking ties arbitrarily. We then define $w^{(k)}(S, \bar{S})=\sum_{e \in E(S, \bar{S}) \backslash F} w_{e}$.

The uniform k-route sparsity of set $S$ is defined to be

$$
\Phi^{(k)}(S)=\frac{w^{(k)}(S, \bar{S})}{\min \{D(S), D(\bar{S})\}},
$$

and the uniform $k$-route sparsity of the graph $G$ is

$$
\Phi^{(k)}(G)=\min _{\substack{S \subset V: \\ D(S), D(\overline{\bar{S}})>0}}\left\{\Phi^{(k)}(S)\right\} .
$$

Similarly, the nonuniform k-route sparsity of $S$ is

$$
\tilde{\Phi}^{(k)}(S)=\frac{w^{(k)}(S, \bar{S})}{D(S, \bar{S})},
$$

and the nonuniform $k$-route sparsity of the graph $G$ is

$$
\tilde{\Phi}^{(k)}(G)=\min _{\substack{S \subset V: \\ D(S, \bar{S})>0}}\left\{\tilde{\Phi}^{(k)}(S)\right\} .
$$

Note that $\Phi^{(1)}(G)=\Phi(G)$ and $\tilde{\Phi}^{(1)}(G)=\tilde{\Phi}(G)$ are the standard uniform and nonuniform sparsest cut values, respectively. We now show that there is an efficient algorithm to find an approximate $k$-route sparsest cut when $k$ is a constant.

THEOREM 2.1. There is an algorithm that, given a graph $G=(V, E)$ with $r$ source-sink pairs and an integer $k$, computes in time $n^{O(k)}$ a cut $S \subseteq V$, with $\Phi^{(k)}(S) \leq \alpha_{\mathrm{ARV}}(r) \cdot \Phi^{(k)}(G)$. Similarly, there is an algorithm that computes in time $n^{O(k)}$ a cut $S$, with $\tilde{\Phi}^{(k)}(S) \leq$ $\alpha_{\mathrm{ALN}}(r) \cdot \tilde{\Phi}^{(k)}(G)$.

PROOF. We start with the uniform $k$-route sparsest cut. We go over all subsets $F \subseteq E$ of $k-1$ edges. For each such subset $F$, we compute the $\alpha_{\mathrm{ARV}}(r)$-approximate sparsest cut in the graph $G \backslash F$ using the algorithm $\mathcal{A}_{\mathrm{ARV}}$ and output the best cut over all such 
subsets $F$. The algorithm for the nonuniform sparsest $k$-route cut is similar, except that we use the algorithm $\mathcal{A}_{\mathrm{ALN}}$ for the nonuniform sparsest cut.

The preceding theorem works well for constant values of $k$. However, when $k$ is superconstant, the running time of the algorithm is no longer polynomial. For such cases, we use a bi-criteria approximation algorithm for the $k$-route sparsest cut problem, summarized in the next theorem.

THEOREM 2.2. There is an efficient algorithm that, given an edge-weighted graph $G=(V, E)$, an integer $k>1$, and a set $D=\left\{\left(s_{i}, r_{i}\right)\right\}_{i=1}^{r}$ of $r$ demand pairs, finds a cut $S \subseteq V$ with $\tilde{\Phi}^{\left(k^{\prime}\right)}(S)=O(\log r) \cdot \tilde{\Phi}^{(k)}(G)$, where $k^{\prime}=C k \log r$ for some absolute constant $C$.

Proof. We use as a subroutine the approximation algorithm of Englert et al. [2010] for the $\ell$-multicut problem. In the $\ell$-multicut problem, we are given a graph $G=(V, E)$ with weights on edges, a set $D$ of $r$ demand pairs, and an integer $\ell$. The goal is to find a minimum-weight subset $E^{\prime} \subseteq E$ of edges such that at least $\ell$ of the demand pairs are disconnected in the graph $G \backslash E^{\prime}$. Englert et al. [2010] give an efficient $O(\log r)$ approximation algorithm for this problem. We denote their algorithm by $\mathcal{A}_{\mathrm{EGK}+}$ and the approximation factor it achieves by $\alpha_{\mathrm{EGK}+}=O(\log r)$.

Let $\left(S^{*}, \bar{S}^{*}\right)$ be the optimal nonuniform sparsest $k$-route cut in $G$, and let $F^{*} \subseteq$ $E_{G}\left(S^{*}, \bar{S}^{*}\right)$ be the subset of the $(k-1)$ most expensive edges in this cut. Then $w\left(E\left(S^{*}, \bar{S}^{*}\right) \backslash F^{*}\right)=\tilde{\Phi}^{(k)}(G) \cdot D\left(S^{*}, \bar{S}^{*}\right)$. Let $W^{*}=w\left(E\left(S^{*}, \bar{S}^{*}\right) \backslash F^{*}\right)$, and let $r^{*}=D\left(S^{*}, \bar{S}^{*}\right)$.

Assume first that our algorithm is given the values of $W^{*}$ and $r^{*}$. We define new edge weights as follows: for each edge $e \in E, \tilde{w}_{e}=\min \left\{w_{e}, W^{*} /(k-1)\right\}$. We use the algorithm $\mathcal{A}_{\mathrm{EGK}}+$ on the resulting instance of the $\ell$-multicut problem with $\ell=r^{*}$. Let $E^{\prime}$ be the output of the algorithm, and let $\mathcal{C}$ be the collection of connected components in $G \backslash E^{\prime}$. We can then find a partition $(S, \bar{S})$ of the vertices of $G$ such that $E(S, \bar{S}) \subseteq E^{\prime}$ and $D(S, \bar{S}) \geq r^{*} / 2$ as follows. We start with an arbitrary partition $(S, \bar{S})$ of the vertices of $G$, where each cluster $C \in \mathcal{C}$ is contained in either $S$ or $\bar{S}$. We then perform several iterations. In each iteration, if there is a cluster $C \in \mathcal{C}$ such that moving all of its vertices to the opposite side of the current cut increases $D(S, \bar{S})$, we move the vertices of $C$ to the opposite side of the cut. It is easy to verify that in the final partition $(S, \bar{S})$, $D(S, \bar{S}) \geq r^{*} / 2$.

Let $(S, \bar{S})$ be the resulting partition, and let $F$ be the set of $2 \alpha_{\mathrm{EGK}+}(r)(k-1)$ most expensive edges of $E(S, \bar{S})$, with respect to the original weights $w_{e}$, breaking ties arbitrarily.

The output of our algorithm is the cut $(S, \bar{S})$. To complete the proof, it is enough to show that $w(E(S, \bar{S}) \backslash F) \leq O(\log r) \cdot \tilde{\Phi}^{(k)}(G) \cdot D(S, \bar{S})$.

Note that the value of the optimal solution to the $\ell$-multicut problem instance is at most

$$
\begin{aligned}
\tilde{w}\left(E\left(S^{*}, \bar{S}^{*}\right)\right) & \leq \tilde{w}\left(E\left(S^{*}, \bar{S}^{*}\right) \backslash F^{*}\right)+\left|F^{*}\right| \cdot \frac{W^{*}}{k-1} \\
& \leq w\left(E\left(S^{*}, \bar{S}^{*}\right) \backslash F^{*}\right)+W^{*}=2 W^{*}
\end{aligned}
$$

Therefore, $\tilde{w}(E(S, \bar{S})) \leq 2 \alpha_{\mathrm{EGK}+}(r) W^{*}$. In particular, $E(S, \bar{S})$ may contain at most $2 \alpha_{\mathrm{EGK}+}(r)(k-1)$ edges $e$ with $\tilde{w}_{e}=W^{*} /(k-1)$, and so all such edges lie in $F$. For edges 
$e \notin F, \tilde{w}_{e}<W^{*} /(k-1)$ must hold, and therefore, $\tilde{w}_{e}=w_{e}$. We conclude that

$$
\begin{aligned}
w(E(S, \bar{S}) \backslash F) & =\tilde{w}(E(S, \bar{S}) \backslash F) \leq \tilde{w}(E(S, \bar{S})) \\
& \leq 2 \alpha_{\mathrm{EGK}+}(r) W^{*}=2 \alpha_{\mathrm{EGK}+}(r) \tilde{\Phi}^{(k)}(G) \cdot r^{*} \\
& \leq O(\log r) \tilde{\Phi}^{(k)}(G) D(S, \bar{S})
\end{aligned}
$$

as required.

Of course, our algorithm does not know the values of $W^{*}$ and $r^{*}$. Instead, we perform the procedure described earlier for all possible values of $r^{\prime} \in\{1, \ldots, r\}$ and (say) all values of $W^{\prime}$ in $\left\{\tau w_{e}: e \in E, 1 \leq \tau \leq|E|\right\}$, then output the best of the cuts found. One of the values of $r^{\prime}$ will be equal to $r^{*}$, and one the values of $W^{\prime}$ will be within a factor of 2 of $W^{*}$ : if $e$ is the most expensive edge in $E\left(S^{*}, \bar{S}^{*}\right) \backslash F^{*}$, and $\tau=\left\lceil W^{*} / w_{e}\right\rceil$, then $W^{*} \leq \tau w_{e} \leq\left(2\left\lfloor W^{*} / w_{e}\right\rfloor\right) w_{e} \leq 2 W^{*}$. For these values of $r^{\prime}$ and $W^{\prime}$, the algorithm will find a cut that satisfies the conditions of the theorem.

Laminar families of minimum cuts. Our main tool in establishing the connection between the values of the $k$-route sparsest cut and the cost of the optimal solution to the $k$-route cut problem is the following lemma, which shows that there is a laminar family of minimum cuts disconnecting the source-sink pairs in the graph $G$. (Recall that a family of sets $S_{1}, \ldots, S_{r} \subseteq V$ is laminar, if for every $i$ and $j$, either $S_{i} \cap S_{j}=\emptyset$, or $S_{i} \subseteq S_{j}$, or $S_{j} \subseteq S_{i}$.)

LEMMA 2.3. There is an efficient algorithm that, given any edge-weighted graph $G=(V, E)$ with a set $D=\left\{\left(s_{i}, t_{i}\right)\right\}_{i=1}^{r}$ of $r$ source-sink pairs, finds a laminar family $\mathcal{S}=\left\{S_{1}, \ldots, S_{r}\right\}$ of vertex subsets such that for all $1 \leq i \leq r$ :

$-\left(S_{i}, V \backslash S_{i}\right)$ is a minimum cut separating $s_{i}$ from $t_{i}$ in $G$, and $-D\left(S_{i}\right) \leq r$ (so $S_{i}$ contains at most half the terminals, counting multiplicities).

Proof. We use a Gomory-Hu tree $T_{G H}$ for the graph $G$ [Gomory and $\mathrm{Hu} 1961$ ]. Recall that it is a weighted tree whose vertex set is $V$. Let $c_{e}$ denote the costs of the edges $e \in E\left(T_{G H}\right)$. Tree $T_{G H}$ has the following key property: for every pair $(u, v) \in V$ of vertices, the value of the minimum cut separating $u$ from $v$ in graph $G$ equals the value of the minimum cut separating $u$ from $v$ in $T_{G H}$. Note that the latter cut contains only one edge-the minimum-cost edge on the unique path connecting $u$ to $v$ in the tree. The existence of a Gomory-Hu tree for any graph $G$ was shown in Gomory and $\mathrm{Hu}$ [1961].

We start with a Gomory-Hu tree $T_{G H}$ for the graph $G$. For each $1 \leq i \leq r$, let $\left(L_{i}, R_{i}\right)$ be a minimum cut separating $s_{i}$ from $t_{i}$ in $T_{G H}$. If $D\left(L_{i}\right)<D\left(R_{i}\right)$, then we set $S_{i}=L_{i}$. If $D\left(R_{i}\right)<D\left(L_{i}\right)$, we set $S_{i}=R_{i}$. Otherwise, if $D\left(R_{i}\right)=D\left(L_{i}\right)$, we let $S_{i}$ to be the side containing the vertex $s_{1}$. We use this tie-breaking rule that enforces consistency across different source-sink pairs later.

This finishes the definition of the family $\mathcal{S}=\left\{S_{1}, \ldots S_{r}\right\}$ of vertex subsets. It is immediate to see that for each $1 \leq i \leq r,\left(S_{i}, V \backslash S_{i}\right)$ is a minimum cut separating $s_{i}$ from $t_{i}$ in $G$ and that $D\left(S_{i}\right) \leq r$. It now only remains to show that $S_{1}, \ldots, S_{r}$ form a laminar family.

Assume for contradiction that for some $i \neq j, S_{i} \cap S_{j} \neq \emptyset$, but $S_{i} \backslash S_{j} \neq \emptyset$, and $S_{j} \backslash S_{i} \neq \emptyset$. Let $e_{i}$ be the unique edge of $T_{G H}$ lying in the cut $\left(S_{i}, V \backslash S_{i}\right)$ in tree $T_{G H}$, and let $e_{j}$ be the unique edge of $T_{G H}$ lying in the cut $\left(S_{j}, V \backslash S_{j}\right)$. Observe that $T_{G H} \backslash\left\{e_{i}, e_{j}\right\}$ consists of three nonempty connected components. Let $C_{1}$ denote the component that is incident on both $e_{i}$ and $e_{j}, C_{2}$ the component incident on $e_{j}$ only, and $C_{3}$ the remaining component. We claim that $S_{i}=C_{1} \cup C_{2}$. Otherwise, since edge $e_{i}$ separates $S_{i}$ from $V \backslash S_{i}$ in $T_{G H}, S_{i}=C_{3}$ must hold. But then either $S_{j}=C_{2}$ and so $S_{i} \cap S_{j}=\emptyset$, or 
$S_{j}=C_{1} \cup C_{3}$ and then $S_{i} \subseteq S_{j}$, a contradiction. Therefore, $S_{i}=C_{1} \cup C_{2}$ and $V \backslash S_{i}=C_{3}$. Similarly, $S_{j}=C_{1} \cup C_{3}$ and $V \backslash S_{j}=C_{2}$.

From the definition of $S_{i}$, either $D\left(S_{i}\right)<D\left(V \backslash S_{i}\right)$, or $D\left(S_{i}\right)=D\left(V \backslash S_{i}\right)$ and $s_{1} \in S_{i}$. Assume first that $D\left(S_{i}\right)<D\left(V \backslash S_{i}\right)$. Then $V \backslash S_{j}=C_{2} \subseteq S_{i}$, and so $D\left(V \backslash S_{j}\right)<D\left(S_{j}\right)$, contradicting the definition of $S_{j}$. We reach a similar contradiction if $D\left(S_{j}\right)<D\left(V \backslash S_{j}\right)$. Therefore, $D\left(S_{i}\right)=D\left(V \backslash S_{i}\right)$ and $D\left(S_{j}\right)=D\left(V \backslash S_{j}\right)$ must hold. In other words, $D\left(V \backslash S_{i}\right)=$ $D\left(C_{3}\right)=r$, and $D\left(V \backslash S_{j}\right)=D\left(C_{2}\right)=r$. Since $C_{2}$ and $C_{3}$ are disjoint, this means that $D\left(C_{1}\right)=0$. But from the definitions of $S_{i}$ and $S_{j}, s_{1} \in S_{i} \cap S_{j}$ must hold, a contradiction.

First algorithmic framework. Most of our algorithms belong to one of two simple algorithmic frameworks. The first framework uses a divide-and-conquer paradigm. We start with the graph $G=(V, E)$ and a set $D$ of $r \geq 1$ demand pairs, and then we find a cut $(S, \bar{S})$ in $G$, with $D(S), D(\bar{S}) \geq 1$. We then select a subset $E_{0} \subseteq E(S, \bar{S})$ of edges to delete and apply the algorithm recursively to the subinstances induced by $G[S]$ and $G[\bar{S}]$. Here, the subinstance induced by $G[S]$ consists of the graph $G[S]$ (the subgraph of $G$ induced by the set $S$ of vertices) and the collection of the original demand pairs $\left(s_{i}, t_{i}\right)$, with both $s_{i}, t_{i} \in S$. The subinstance induced by $G[\bar{S}]$ is defined similarly. Let $E_{1}$ and $E_{2}$ be the solutions returned by the two recursive calls, respectively. The final solution is $E^{\prime}=E_{0} \cup E_{1} \cup E_{2}$. The specific cut $(S, \bar{S})$, and the subsets $E_{0} \subseteq E(S, \bar{S})$ of edges computed will differ from algorithm to algorithm, and we will need to select them in a way that ensures the feasibility of the final solution. However, the analysis of the solution cost is similar in all of these algorithms and is summarized in the following theorem.

THEOREM 2.4. Let $\mathcal{A}$ be any algorithm in the preceding framework, and assume that the algorithm guarantees that $w\left(E_{0}\right) \leq \frac{\alpha \cdot \mathrm{OPT}}{r} \cdot \min \{D(S), D(\bar{S})\}$ for some factor $\alpha$. Then $w\left(E^{\prime}\right) \leq 4 \alpha \ln (1+r) \cdot$ OPT.

Proof. The proof is by induction on $r$. If $r=1$ then $E^{\prime}=E_{0}$, and the statement trivially holds. Assume now that the statement holds for instances with fewer than $r$ demand pairs for some $r>1$. Consider the cut $(S, \bar{S})$ computed by the algorithm $\mathcal{A}$ on the current instance. Let $a$ be the number of demand pairs $\left(s_{i}, t_{i}\right)$ with $s_{i}, t_{i} \in S$, let $b$ be the number of demand pairs $\left(s_{i}, t_{i}\right)$ with $s_{i}, t_{i} \in \bar{S}$, and assume without loss of generality that $a \geq b$. Then $D(S) \geq 2 a, D(\bar{S}) \geq 2 b$, and so $D(S) \leq 2 r-D(\bar{S}) \leq 2(r-b)$ and $D(\bar{S}) \leq 2(r-a)$. Therefore,

$$
w\left(E_{0}\right) \leq \frac{\alpha \cdot \mathrm{OPT}}{r} \cdot \min \{D(S), D(\bar{S})\} \leq \frac{2 \alpha \cdot \mathrm{OPT}}{r}(r-a) .
$$

The optimal solutions to the EC-kRC instances on graphs $G[S]$ and $G[\bar{S}]$ have costs at most $w\left(E^{*} \cap E(S)\right)$ and $w\left(E^{*} \cap E(\bar{S})\right)$, respectively. By the induction hypothesis, the total cost of solutions $E_{1}$ and $E_{2}$ on graphs $G[S]$ and $G[\bar{S}]$ is at most

$$
\begin{aligned}
4 \alpha w & \left(E^{*} \cap E(S)\right) \ln (1+a)+4 \alpha w\left(E^{*} \cap E(\bar{S})\right) \ln (1+b) \\
& \leq 4 \alpha\left(w\left(E^{*} \cap E(S)\right)+w\left(E^{*} \cap E(\bar{S})\right)\right) \ln (1+a) \\
& \leq 4 \alpha \cdot \mathrm{OPT} \cdot \ln (1+a) .
\end{aligned}
$$

The total solution cost is then bounded by

$$
\begin{aligned}
w\left(E^{\prime}\right) & \leq 4 \alpha \cdot \text { OPT } \cdot \ln (1+a)+\frac{2 \alpha \cdot \text { OPT }}{r}(r-a) \\
& \leq 4 \alpha \cdot \text { OPT }\left(\ln (1+a)+\frac{r-a}{2 r}\right) .
\end{aligned}
$$


The theorem follows from the following inequality:

$$
\begin{aligned}
\ln (1+a)+\frac{r-a}{2 r} & =\ln (1+r)+\ln \left(\frac{1+a}{1+r}\right)+\frac{r-a}{2 r} \\
& \leq \ln (1+r)-\frac{r-a}{1+r}+\frac{r-a}{2 r} \\
& \leq \ln (1+r),
\end{aligned}
$$

where we have used the fact that $\ln \left(\frac{1+a}{1+r}\right)=\ln \left(1-\frac{r-a}{1+r}\right) \leq-\frac{r-a}{1+r}$, since $\ln (1+x) \leq x$ for all $x>-1$.

Second algorithmic framework. The first algorithmic framework has some limitations. Specifically, we can only use it in scenarios where there is a cheap collection $E^{\prime}$ of edges (with cost roughly comparable to OPT), whose removal decomposes our instance $G$ into two disjoint subinstances, $G[S], G[\bar{S}]$, which can then be solved separately. This is the case for the uniform EC-kRC, and the nonuniform EC-kRC and VC-kRC when $k=2$. For higher values of $k$ in the nonuniform setting, such a decomposition may not exist. Instead, we use the following framework. Given a graph $G$ and a set $D$ of $r \geq 1$ demand pairs, we find a collection $E_{0}$ of edges to delete, together with a subset $D_{0}$ of demand pairs to remove, where $\left|D_{0}\right| \geq 1$. We then solve the problem recursively on the graph $G^{\prime}=G \backslash E_{0}$ and the set $D \backslash D_{0}$ of the remaining demand pairs. Let $E_{1}$ be the subset of edges returned by the recursive call. Then the solution computed by the algorithm is $E^{\prime}=E_{0} \cup E_{1}$. The specific subset $E_{0}$ of edges to remove and the subset $D_{0}$ of demands will again be computed differently by each algorithm in a way that ensures the final solution is feasible. The analysis of the solution cost of such algorithms is summarized in the next theorem.

THEOREM 2.5. Let $\mathcal{A}$ be any algorithm in the preceding framework, and assume that we are guaranteed that $w\left(E_{0}\right) \leq \alpha$. OPT $\cdot \frac{\left|D_{0}\right|}{r}$ for some factor $\alpha$. Then $w\left(E^{\prime}\right) \leq$ $2 \alpha \ln (1+r)$. OPT.

Proof. The proof is by induction on $r$. If $r=1$ then $E^{\prime}=E_{0}$, and the statement trivially holds. Assume now that the statement holds for instances with fewer than $r$ demand pairs for some $r>1$. We prove the theorem for instances with $r$ demand pairs. Let $a=\left|D_{0}\right|$. Then by the induction hypothesis, $w\left(E_{1}\right) \leq 2 \alpha \cdot$ OPT $\cdot \ln (1+r-a)$. Therefore,

$$
\begin{aligned}
w\left(E^{\prime}\right) & \leq 2 \alpha \cdot \text { OPT } \cdot \ln (1+r-a)+\alpha \cdot \text { OPT } \cdot \frac{a}{r} \\
& =2 \alpha \cdot \text { OPT }\left(\ln (r+1)+\ln \left(\frac{1+r-a}{r+1}\right)+\frac{a}{2 r}\right) \\
& \leq 2 \alpha \cdot \text { OPT }\left(\ln (r+1)-\frac{a}{r+1}+\frac{a}{2 r}\right) \\
& \leq 2 \alpha \ln (r+1) \text { OPT. }
\end{aligned}
$$

\section{UNIFORM EC-kRC}

This section is dedicated to proving Theorem 1.1. We first show an $O\left(k \log ^{1.5} r\right)$ approximation algorithm and provide a bi-criteria algorithm later. Recall that we are given an unweighted graph $G=(V, E)$, a set $\left\{\left(s_{i}, t_{i}\right)\right\}_{i=1}^{r}$ of demand pairs, and an integer $k$. Our goal is to find a collection $E^{\prime}$ of $O\left(k \log ^{3 / 2} r\right)$. OPT edges such that for each demand pair $\left(s_{i}, t_{i}\right)$, there are at most $(k-1)$ edge-disjoint paths in graph $G \backslash E^{\prime}$ connecting them. 
Input: An unweighted graph $G=(V, E)$ with demand pairs $\left\{\left(s_{i}, t_{i}\right)\right\}_{1 \leq i \leq r}$

Output: A set $E^{\prime}$ of edges such that each pair $\left(s_{i}, t_{i}\right)$ has at most $(k-1)$ edge-disjoint paths connecting $s_{i}$ to $t_{i}$ in $G \backslash E^{\prime}$.

(1) If $r=0$ return $E^{\prime}=\emptyset$.

(2) Find a partition $(S, \bar{S})$ of $V$ using the algorithm $\mathcal{A}_{\mathrm{ARV}}$, with $\Phi(S) \leq \alpha_{\mathrm{ARV}}(r) \cdot \Phi(G)$.

(3) Let $E_{0}=E(S, \bar{S}), G^{\prime}=G \backslash E_{0}$.

(4) Remove all demand pairs $\left(s_{i}, t_{i}\right)$ that are no longer $k$-edge connected in $G^{\prime}$.

(5) Solve the instances induced by $G[S]$ and $G[V \backslash S]$ recursively, to obtain solutions $E_{1}^{\prime}$ and $E_{2}^{\prime}$, respectively.

(6) Return $E^{\prime}=E_{1}^{\prime} \cup E_{2}^{\prime} \cup E_{0}$.

Fig. 1. Approximation algorithm for uniform EC-kRC.

We assume without loss of generality that each source-sink pair $\left(s_{i}, t_{i}\right)$ is at least $k$-edge connected in the current graph $G$. Our algorithm views the graph $G$ as an instance of the uniform sparsest cut problem. We use the algorithm $\mathcal{A}_{\mathrm{ARV}}$ to find a partition $(S, \bar{S})$ of $V$ with $\Phi(S) \leq \alpha_{\mathrm{ARV}}(r) \cdot \Phi(G)$, add the edges in $E(S, \bar{S})$ to the solution $E^{\prime}$, and delete the demand pairs $\left(s_{i}, t_{i}\right)$ that are no longer $k$-edge connected from the list of source-sink pairs. Notice that each remaining source-sink pair must be contained either in $S$ or in $\bar{S}$. We then recursively solve the EC-kRC problem on the subinstances induced by $G[S]$ and $G[\bar{S}]$. The algorithm is summarized in Figure 1.

The heart of the analysis of the algorithm is the following theorem, which relates the value of the uniform sparsest cut in graph $G$ to the value OPT of the optimal solution for EC-kRC.

Theorem 3.1. Suppose that we are given an unweighted graph $G=(V, E)$ with $r$ source-sink pairs $\left\{\left(s_{i}, t_{i}\right)\right\}_{i=1}^{r}$ such that for each pair $\left(s_{i}, t_{i}\right)$, there are at least $k$ edgedisjoint paths connecting $s_{i}$ to $t_{i}$ in $G$, and let OPT be the cost of the optimal solution of EC-kRC on $G$. Then $\Phi(G) \leq \frac{2 k}{r}$. OPT.

Proof. Consider the graph $H=G \backslash E^{*}$. We use Lemma 2.3 with edge weights $w_{e}=1$ on graph $H$ to obtain the laminar family $\mathcal{S}=\left\{S_{i}\right\}_{i=1}^{r}$ of vertex subsets. Consider all maximal sets in the laminar family - that is, sets $S_{i}$ that are not contained in other sets. Assume without loss of generality that these sets are $S_{1}, \ldots, S_{q}$, for some $q \leq r$. Then $\sum_{i=1}^{q} D\left(S_{i}\right) \geq r$ must hold. Note that for each $i,\left|E_{H}\left(S_{i}, V \backslash S_{i}\right)\right| \leq k-1$ since $s_{i}$ and $t_{i}$ are not $k$-edge connected in $H$, and $\left(S_{i}, V \backslash S_{i}\right)$ is a minimum cut separating $s_{i}$ from $t_{i}$ in $H$. On the other hand, $\left|E_{G}\left(S_{i}, V \backslash S_{i}\right)\right| \geq k$ since $s_{i}$ and $t_{i}$ are $k$-edge connected in $G$. Therefore,

$$
\begin{aligned}
\left|E_{G}\left(S_{i}, V \backslash S_{i}\right)\right|= & \left|E_{H}\left(S_{i}, V \backslash S_{i}\right)\right| \\
& \quad+\left|E_{G}\left(S_{i}, V \backslash S_{i}\right) \cap E^{*}\right| \\
\leq & (k-1)+\left|E_{G}\left(S_{i}, V \backslash S_{i}\right) \cap E^{*}\right| \\
\leq & k\left|E_{G}\left(S_{i}, V \backslash S_{i}\right) \cap E^{*}\right| .
\end{aligned}
$$

Note that every edge $e \in E^{*}$ belongs to at most two cuts $E_{G}\left(S_{i}, V \backslash S_{i}\right)$ and $E_{G}\left(S_{j}, V \backslash S_{j}\right)$, as all sets $S_{1}, \ldots, S_{q}$ are disjoint. Therefore,

$$
\begin{aligned}
\sum_{i=1}^{q}\left|E_{G}\left(S_{i}, V \backslash S_{i}\right)\right| & \leq \sum_{i=1}^{q} k\left|E_{G}\left(S_{i}, V \backslash S_{i}\right) \cap E^{*}\right| \\
& \leq 2 k \cdot \text { OPT. }
\end{aligned}
$$


On the other hand,

$$
\begin{aligned}
\sum_{i=1}^{q}\left|E_{G}\left(S_{i}, V \backslash S_{i}\right)\right| & =\sum_{i=1}^{q} \Phi\left(S_{i}\right) \cdot D\left(S_{i}\right) \\
& \geq \sum_{i=1}^{q} \Phi(G) \cdot D\left(S_{i}\right) \\
& \geq r \cdot \Phi(G) .
\end{aligned}
$$

We conclude that $\Phi(G) \leq 2 k \cdot \mathrm{OPT} / r$.

We now analyze the algorithm. Since the algorithm removes a demand pair $\left(s_{i}, t_{i}\right)$ only when $s_{i}$ and $t_{i}$ are no longer $k$-edge connected and terminates when all demand pairs are removed, the algorithm is guaranteed to find a feasible solution to the problem. To bound the solution cost, note that

$$
\begin{aligned}
\left|E_{0}\right| & =\Phi(S) \cdot \min \{D(S), D(\bar{S})\} \\
& \leq \alpha_{\mathrm{ARV}}(r) \cdot \Phi(G) \cdot \min \{D(S), D(\bar{S})\} \\
& \leq \frac{2 k \alpha_{\mathrm{ARV}}(r)}{r} \cdot \mathrm{OPT} \cdot \min \{D(S), D(\bar{S})\}
\end{aligned}
$$

We can now use Theorem 2.4 with $\alpha=2 k \alpha_{\mathrm{ARV}}(r)$ to conclude that $\left|E^{\prime}\right|=O\left(k \alpha_{\mathrm{ARV}}(r)\right.$ $\log r) \mathrm{OPT}=O\left(k \log ^{3 / 2} r\right) \mathrm{OPT}$.

Bi-criteria approximation algorithm. We now slightly modify the algorithm from Figure 1 to obtain a $\left(1+\delta, O\left(\frac{1}{\delta} \log ^{1.5} r\right)\right)$-bi-criteria approximation algorithm for any constant $0<\delta<1$. The algorithm works exactly as before, except that it removes a demand pair $\left(s_{i}, t_{i}\right)$ in step 4 if and only if $s_{i}$ and $t_{i}$ are no longer $(1+\delta) k$ edge connected. We also assume without loss of generality that in the original instance $G$, every demand pair $\left(s_{i}, t_{i}\right)$ has at least $(1+\delta) k$ edge-disjoint paths connecting $s_{i}$ to $t_{i}$. As before, it is straightforward to verify that if $E^{\prime}$ is the final solution produced by the algorithm, then each demand pair $\left(s_{i}, t_{i}\right)$ pair has fewer than $(1+\delta) k$ edge-disjoint paths connecting them in $G \backslash E^{\prime}$. To bound the solution cost, we prove the following analogue of Theorem 3.1.

THeOREM 3.2. Suppose that we are given an unweighted graph $G$ with $r$ demand pairs $\left\{\left(s_{i}, t_{i}\right)\right\}_{i=1}^{r}$, where for each pair $\left(s_{i}, t_{i}\right)$ there are at least $(1+\delta) k$ edge-disjoint paths connecting $s_{i}$ to $t_{i}$ in $G$. Then $\Phi(G) \leq \frac{2 \mathrm{OPT}}{r} \cdot(1+1 / \delta)$.

Proof. As before, we compute the laminar family of minimum cuts in graph $H=$ $G \backslash E^{*}$, using Lemma 2.3, and we consider the collection of all maximal sets in this family. Assume without loss of generality that it is $\left\{S_{1}, \ldots, S_{q}\right\}$, for $q \leq r$, and recall that $\sum_{i=1}^{q} D\left(S_{i}\right) \geq r$. As before, for each $1 \leq i \leq q,\left|E_{G}\left(S_{i}, V \backslash S_{i}\right)\right| \leq(k-1)+\mid E_{G}\left(S_{i}, V \backslash\right.$ $\left.S_{i}\right) \cap E^{*} \mid$. Since $\left|E_{G}\left(S_{i}, V \backslash S_{i}\right)\right| \geq(1+\delta) k$, we get that $\left|E_{G}\left(S_{i}, V \backslash S_{i}\right) \cap E^{*}\right| \geq \delta k$, and so $(k-1) \leq\left|E_{G}\left(S_{i}, V \backslash S_{i}\right) \cap E^{*}\right| / \delta$. We get that

$$
\begin{aligned}
\left|E_{G}\left(S_{i}, V \backslash S_{i}\right)\right| & \leq(k-1)+\left|E_{G}\left(S_{i}, V \backslash S_{i}\right) \cap E^{*}\right| \\
& \leq(1+1 / \delta)\left|E_{G}\left(S_{i}, V \backslash S_{i}\right) \cap E^{*}\right|
\end{aligned}
$$


On the other hand, $\left|E_{G}\left(S_{i}, V \backslash S_{i}\right)\right| \geq \Phi(G) \cdot D\left(S_{i}\right)$. Summing up over all $1 \leq i \leq q$, we get that

$$
\begin{aligned}
2 \mathrm{OPT} & \geq \sum_{i=1}^{q}\left|E_{G}\left(S_{i}, V \backslash S_{i}\right) \cap E^{*}\right| \\
& \geq \frac{\delta}{\delta+1} \sum_{i=1}^{q}\left|E_{G}\left(S_{i}, V \backslash S_{i}\right)\right| \\
& \geq \frac{\delta}{\delta+1} \Phi(G) \sum_{i=1}^{q} D\left(S_{i}\right) \geq \frac{\delta}{\delta+1} \Phi(G) \cdot r .
\end{aligned}
$$

We conclude that $\Phi(G) \leq \frac{2 \mathrm{OPT}}{r}(1+1 / \delta)$.

To bound the final solution cost, observe that

$$
\begin{aligned}
\left|E_{0}\right| & =\Phi(S) \cdot \min \{D(S), D(\bar{S})\} \\
& \leq \alpha_{\mathrm{ARV}}(r) \cdot \Phi(G) \cdot \min \{D(S), D(\bar{S})\} \\
& \leq \frac{2 \mathrm{OPT} \alpha_{\mathrm{ARV}}(r)}{r} \cdot(1+1 / \delta) \cdot \min \{D(S), D(\bar{S})\} .
\end{aligned}
$$

We now use Theorem 2.4 with $\alpha=2 \alpha_{\mathrm{ARV}}(r)(1+1 / \delta)$ to conclude that $\left|E^{\prime}\right|=$ $O\left(\alpha_{\mathrm{ARV}}(r) \log r / \delta\right) \mathrm{OPT}=O\left(\log ^{1.5} r / \delta\right)$ OPT when $0<\delta<1$.

This concludes the proof of Theorem 1.1 .

\section{NONUNIFORM EC-kRC}

In this section, we prove Theorem 1.2. We start with a $\left(2, \tilde{O}\left(\log ^{2.5} r\right)\right)$-bi-criteria algorithm with running time $n^{O(k)}$, and we show an algorithm whose running time is polynomial in $n$ and $k$ later.

Abusing the notation, for each cut $(S, \bar{S})$ in graph $G$, we denote by $D(S, \bar{S})$ both the set of the demand pairs $\left(s_{i}, t_{i}\right)$ with $\left|\left\{s_{i}, t_{i}\right\} \cap S\right|=1$ and the number of such pairs.

\subsection{A (2, Õ $\left.\left(\log ^{5 / 2} r\right)\right)$ Bi-Criteria Approximation in Time $n^{O(k)}$}

We cannot employ the first algorithmic framework for EC-kRC on weighted graphs. A natural approach in using it would be to find an appropriately defined sparse cut $(S, \bar{S})$, remove all but $k-1$ most expensive edges of this cut, and then recursively solve the problem on instances $G[S]$ and $G[\bar{S}]$. Let $E_{0}$ be the subset of edges removed, and let $G^{\prime}=G \backslash E_{0}$ be the remaining graph. This approach does not work because it is possible that a demand pair $\left(s_{i}, t_{i}\right)$ with both $s_{i}, t_{i} \in S$ is connected by a path that visits $G[\bar{S}]$ in graph $G^{\prime}$. So if we solve the problem recursively on $G[S]$ and $G[\bar{S}]$, then the combined solution is not necessarily a feasible solution to the problem. On the other hand, if instead we remove all or almost all edges of $E(S, \bar{S})$, then the resulting solution cost may be too high. Therefore, we employ the second algorithmic framework instead.

We assume without loss of generality that in the input graph $G$, each demand pair $\left(s_{i}, t_{i}\right)$ has at least $(2 k-1)$ edge-disjoint paths connecting them. Our algorithm, summarized in Figure 2 , starts by finding an approximate nonuniform $(2 k-1)$-route sparse cut $(S, \bar{S})$ in $G$, using Theorem 2.1. In other words, $\tilde{\Phi}^{(2 k-1)}(S) \leq \alpha_{\mathrm{ALN}}(r) \tilde{\Phi}^{(2 k-1)}(G)$. Let $F$ be the set of the $(2 k-2)$ most expensive edges of $E(S, \bar{S})$, let $E_{0}=E(S, \bar{S}) \backslash F$, and let $G^{\prime}=G \backslash E_{0}$. We remove all demand pairs that are no longer $(2 k-1)$ connected in $G^{\prime}$ (we denote the set of these demand pairs by $D_{0}$ ) and then recursively solve the resulting instance. 
Input: A weighted graph $G=(V, E)$ with a set $D=\left\{\left(s_{i}, t_{i}\right)\right\}_{1 \leq i \leq r}$ of demand pairs, and edge weights $\left\{w_{e}\right\}_{e \in E}$.

Output: A set $E^{\prime}$ of edges such that each demand pair $\left(s_{i}, t_{i}\right)$ is no longer $(2 k-1)$ edge connected in $G \backslash E^{\prime}$.

(1) If $r=0$ return $E^{\prime}=\emptyset$.

(2) Find an approximate nonuniform $(2 k-1)$-route sparsest cut $(S, \bar{S})$ with $\tilde{\Phi}^{(2 k-1)}(S) \leq \alpha_{\mathrm{ALN}}(r) \cdot \tilde{\Phi}^{(2 k-1)}(G)$, using Theorem 2.1. Let $F$ be the set of the $(2 k-2)$ most expensive edges in $E(S, \bar{S})$, breaking ties arbitrarily.

(3) Let $E_{0}=E(S, \bar{S}) \backslash F, G^{\prime}=G \backslash E_{0}$, and let $D_{0}$ be the set of all demand pairs that are no longer $(2 k-1)$-connected in $G^{\prime}$.

(4) Recursively solve the problem on $G^{\prime}$ with the demand set $D \backslash D_{0}$ to obtain a solution $E_{1}$.

(5) Return $E^{\prime}=E_{0} \cup E_{1}$.

Fig. 2. A bi-criteria approximation algorithm for nonuniform EC-kRC in time $n^{O(k)}$.

It is immediate to verify that the algorithm returns a feasible solution. The running time of the algorithm is dominated by computing an approximate $(2 k-1)$-route sparsest cut and is therefore bounded by $n^{O(k)}$ from Theorem 2.1. To bound the solution cost, we use the following theorem that relates the value of $\tilde{\Phi}^{(2 k-1)}(G)$ to OPT.

THEOREM 4.1. Suppose that we are given a graph $G=(V, E)$ with edge weights $w_{e}$, and a set $D=\left\{\left(s_{i}, t_{i}\right)\right\}_{i \in[r]}$ of $r$ demand pairs, where every pair $\left(s_{i}, t_{i}\right)$ has at least $(2 k-1)$ edge-disjoint paths connecting $s_{i}$ to $t_{i}$ in $G$. Then OPT $=\Omega\left(\frac{r}{\log r}\right) \tilde{\Phi}^{(2 k-1)}(G)$.

Proof. Consider the graph $H=G \backslash E^{*}$. Let $\mathcal{S}=\left\{S_{1}, \ldots, S_{r}\right\}$ be the laminar family of minimum cuts in $H$, guaranteed by Lemma 2.3. Recall that for all $1 \leq i \leq r$, $\left|E_{H}\left(S_{i}, V \backslash S_{i}\right)\right| \leq k-1$. We need the following lemma.

LEMMA 4.2. We can efficiently find a collection $\mathcal{P}$ of mutually disjoint vertex subsets such that

-For each $U \in \mathcal{P}, D(U) \leq r$;

-For each $U \in \mathcal{P},\left|E_{H}(U, \bar{U})\right| \leq 2(k-1)$; and

$-\sum_{U \in \mathcal{P}} D(U, \bar{U}) \geq \frac{r}{8 \log r}$.

Proof. We will define each set $U \in \mathcal{P}$ to be either some set $S \in \mathcal{S}$, or a difference of two sets, $U=S \backslash S^{\prime}$, for $S, S^{\prime} \in \mathcal{S}$. Since for each set $S \in \mathcal{S}, D(S) \leq r$, this will ensure the first condition. Since $\left|E_{H}(U, \bar{U})\right| \leq\left|E_{H}(S, \bar{S})\right|+\left|E_{H}\left(S^{\prime}, \bar{S}^{\prime}\right)\right| \leq 2(k-1)$, this will also ensure the second condition.

We now turn to define the sets $U \in \mathcal{P}$ so that the third condition is also satisfied. For simplicity, if collection $\mathcal{S}$ contains identical sets, we discard them, keeping at most one copy of each set in $\mathcal{S}$. Recall that for each set $S \in \mathcal{S}, D(S, \bar{S})$ is the set of all demand pairs $\left(s_{j}, t_{j}\right)$ with $\left|\left\{s_{j}, t_{j}\right\} \cap S\right|=1$. Let $D^{\prime}(S, \bar{S})$ be the union of $D\left(S^{\prime}, \bar{S}^{\prime}\right)$ for all sets $S^{\prime} \in \mathcal{S}$ where $S^{\prime} \subset S$, and let $q(S)=\left|D(S, \bar{S}) \backslash D^{\prime}(S, \bar{S})\right|$

We partition the family $\mathcal{S}$ into subsets $\mathcal{S}_{x}$, for $1 \leq x \leq\left\lfloor\log _{2} r\right\rfloor+1$, as follows. Collection $\mathcal{S}_{x}$ contains all sets $S \in \mathcal{S}$ with $2^{x-1} \leq q(S)<2^{x}$. Since $\sum_{S \in \mathcal{S}} q(S)=r$, there is at least one index $x^{*}$, for which $\sum_{S \in \mathcal{S}_{x^{*}}} q(S) \geq \frac{r}{2 \log r}$. Fix any such index $x^{*}$.

Consider the decomposition forest $\mathcal{F}$ for the sets in $\mathcal{S}_{x^{*}}$. The nodes of the forest are the sets in $\mathcal{S}_{x^{*}}$, and for a pair $S, S^{\prime} \in \mathcal{S}_{x^{*}}$, set $S$ is the parent of $S^{\prime}$ if and only if $S^{\prime} \subset S$, and there is no other set $S^{\prime \prime} \in \mathcal{S}_{x^{*}}$ with $S^{\prime} \subset S^{\prime \prime} \subset S$. Let $\mathcal{S}^{\prime} \subseteq \mathcal{S}_{x^{*}}$ be the collection of sets that have at most one child in this forest. We are now ready to define the collection 
$\mathcal{P}$ of vertex subsets. If $S \in \mathcal{S}^{\prime}$ is a leaf in $\mathcal{F}$, then we add $S$ to $\mathcal{P}$. Otherwise, if $S$ is a nonleaf set in $\mathcal{S}^{\prime}$, and $S^{\prime}$ is the unique child of $S$ in $\mathcal{F}$, then we add $S \backslash S^{\prime}$ to $\mathcal{P}$.

It now only remains to prove that $\sum_{U \in \mathcal{P}} D(U, \bar{U}) \geq \frac{r}{8 \log r}$. To do so, observe that $\left|\mathcal{S}^{\prime}\right| \geq$ $\left|\mathcal{S}_{x^{*}}\right| / 2$, and recall that for each $S \in \mathcal{S}_{x^{*}}, 2^{x^{*}-1} \leq q(S)<2^{x^{*}}$. Therefore, $\sum_{U \in \mathcal{P}} D(U, \bar{U}) \geq$ $\sum_{S \in \mathcal{S}^{\prime}} q(S) \geq \frac{\left|\mathcal{S}_{x^{*}}\right|}{2} \cdot 2^{x^{*}-1}=\left|\mathcal{S}_{x^{*}}\right| \cdot 2^{x^{*}-2}$. On the other hand, $\sum_{S \in \mathcal{S}_{x^{*}}} q(S) \geq \frac{r}{2 \log r}$, and so $\left|\mathcal{S}_{x^{*}}\right| \geq \frac{r}{2^{x^{*}+1} \log r}$. We conclude that $\sum_{U \in \mathcal{P}} D(U, \bar{U}) \geq \frac{r}{8 \log r}$.

Let $\mathcal{P}$ be the set family computed by Lemma 4.2. Clearly, for each $U \in \mathcal{P}$,

$$
\begin{aligned}
w^{(2 k-1)}(U, \bar{U}) & =\tilde{\Phi}^{(2 k-1)}(U) \cdot D(U, \bar{U}) \\
& \geq \tilde{\Phi}^{(2 k-1)}(G) \cdot D(U, \bar{U}) .
\end{aligned}
$$

On the other hand, since $\left|E_{H}(U, \bar{U})\right| \leq 2 k-2, w\left(E^{*} \cap E_{G}(U, \bar{U})\right) \geq w^{(2 k-1)}(U, \bar{U})$ must hold. Therefore,

$$
\sum_{U \in \mathcal{P}} w^{(2 k-1)}(U, \bar{U}) \leq \sum_{U \in \mathcal{P}} w\left(E^{*} \cap E_{G}(U, \bar{U})\right) \leq 2 \mathrm{OPT} .
$$

Combining Equations (2) and (3), we get that

$$
\begin{aligned}
2 \mathrm{OPT} & \geq \sum_{U \in \mathcal{P}} w^{(2 k-1)}(U, \bar{U}) \geq \tilde{\Phi}^{(2 k-1)}(G) \sum_{U \in \mathcal{P}} D(U, \bar{U}) \\
& \geq \tilde{\Phi}^{(2 k-1)}(G) \cdot \frac{r}{8 \log r} .
\end{aligned}
$$

Therefore, OPT $=\Omega\left(\frac{r}{\log r}\right) \tilde{\Phi}^{(2 k-1)}(G)$.

To bound the cost $w\left(E^{\prime}\right)$ of the solution, we note that $D(S, \bar{S}) \subseteq D_{0}$, and so

$$
\begin{aligned}
w\left(E_{0}\right)=w^{(2 k-1)}(S, \bar{S}) & =\tilde{\Phi}^{(2 k-1)}(S) \cdot D(S, \bar{S}) \\
& \leq \alpha_{\mathrm{ALN}}(r) \cdot \tilde{\Phi}^{(2 k-1)}(G) \cdot\left|D_{0}\right| \\
& =O\left(\alpha_{\mathrm{ALN}}(r) \log r\right) \cdot \frac{\left|D_{0}\right|}{r} \cdot \text { OPT. }
\end{aligned}
$$

We can now use Theorem 2.5 with $\alpha=O\left(\alpha_{\mathrm{ALN}}(r) \log r\right)$ to conclude that $w\left(E^{\prime}\right)=$ $O\left(\alpha_{\mathrm{ALN}}(r) \log ^{2} r\right)=O\left(\log ^{2.5} r \log \log r\right)$.

\subsection{A Polynomial-Time Bi-Criteria Approximation Algorithm}

In this section, we extend the algorithm from Section 4.1 to handle higher values of $k$ in polynomial time. Notice that the bottleneck in the algorithm from Section 4.1 is computing the approximate multiroute sparsest cut via Theorem 2.1 , which is done in time $n^{O(k)}$. We use Theorem 2.2 instead, which gives an efficient algorithm for computing the $k$-route sparsest cut, albeit with somewhat weaker guarantees.

Our algorithm is identical to the algorithm in Figure 2, except for the following changes. First, in step 2, we use Theorem 2.2 to find a cut $S$ such that $\tilde{\Phi}^{\left(k^{\prime}\right)}(S)=O(\log r) \tilde{\Phi}^{(2 k-1)}(G)$, where $k^{\prime}=C(2 k-1) \log r$ and $C$ is the constant from Theorem 2.2. We let $F$ be the $k^{\prime}-1$ most expensive edges in $E(S, \bar{S})$, and we let $D_{0}$ be the set of all demand pairs that are no longer $k^{\prime}$-connected in $G^{\prime}$. Note that $\tilde{\Phi}^{\left(k^{\prime}\right)}(S) \leq O(\log r) \tilde{\Phi}^{(2 k-1)}(G)$ by Theorem 2.2 and $\tilde{\Phi}^{(2 k-1)}(G) \leq O\left(\frac{\log r}{r}\right)$ OPT by Theorem 4.1. Therefore, $w\left(E_{0}\right) \leq \tilde{\Phi}^{\left(k^{\prime}\right)}(S) \cdot D(S, \bar{S}) \leq O(\log r) \tilde{\Phi}^{(2 k-1)}(G) \cdot D_{0} \leq O\left(\frac{\log ^{2} r}{r}\right) \mathrm{OPT} \cdot D_{0}$. 
Using Theorem 2.5 with $\alpha=O\left(\log ^{2} r\right)$, we get that the algorithm returns a bi-criteria $\left(O(\log r), O\left(\log ^{3} r\right)\right)$-approximate solution to the problem.

\section{VERTEX CONNECTIVITY}

In this section, we extend our approximation algorithms for EC-kRC to handle vertexconnectivity and prove Theorem 1.3. We start by extending some of our technical definitions and results to the vertex-connectivity setting.

Let $(s, t)$ be any pair of vertices in graph $G$, and let $\Delta \subseteq V$ be any subset of vertices. We say that $\Delta$ is a separator for $s$ and $t$, or that $\Delta$ separates $s$ and $t$, iff $s, t \notin \Delta$, and $s$ and $t$ belong to two distinct connected components of $V \backslash \Delta$. We say that $\Delta$ is a minimum cost separator for $(s, t)$ if and only if for each subset $\Delta^{\prime}$ separating $s$ from $t,|\Delta| \leq\left|\Delta^{\prime}\right|$. Given any pair $S, T \subseteq V$ of vertex subsets, let $E(S, T)$ be the set of edges with one endpoint in $S$ and the other endpoint in $T$. Similarly, we say that $\Delta$ separates $S$ from $T$ if and only if $S \cap \Delta=\emptyset, T \cap \Delta=\emptyset, S \cap T=\emptyset$, and $E(S, T)=\emptyset$. Notice that in general, $G \backslash \Delta$ may contain more than two connected components. A vertex cut in graph $G=(V, E)$ is a tri-partition $(S, \Delta, T)$ of $V$, where $E(S, T)=\emptyset$. For any subset $\Delta \subseteq V$, we will sometimes refer to $|\Delta|$ as the cost of $\Delta$.

We start with the following lemma, which is an analogue of Lemma 2.3 for vertex cuts. For technical reasons, it is more convenient to state it for graphs with costs on vertices. Given a graph $G=(V, E)$ with $\operatorname{costs} c_{v}$ on vertices $v \in V$, a cost of a subset $\Delta \subseteq V$ of vertices is $\sum_{v \in \Delta} c_{v}$.

LemMa 5.1. Suppose that we are given a graph $G=(V, E)$ with costs $c_{v}$ on vertices $v \in V$ and a set $\left\{\left(s_{1}, t_{1}\right),\left(s_{2}, t_{2}\right), \ldots,\left(s_{r}, t_{r}\right)\right\}$ of $r$ demand pairs. Let $\mathcal{T}$ be the set of all vertices participating in the demand pairs. Suppose additionally that for every demand pair $\left(s_{i}, t_{i}\right)$, for every minimum-cost separator $\Delta$ for $\left(s_{i}, t_{i}\right), \Delta \cap \mathcal{T}=\emptyset$. Then there exists a family of vertex cuts $\left(S_{i}, \Delta_{i}, T_{i}\right)$ such that

(1) For every $i \in\{1, \ldots, r\}, \Delta_{i}$ is a minimum cost separator for $\left(s_{i}, t_{i}\right)$ (note that $s_{i}$ may belong either to $S_{i}$ or $T_{i}$ ); and

(2) Sets $\left\{S_{i}\right\}_{i=1}^{r}$ form a laminar family.

Proof. We start by considering a "nondegenerate" case, where every subset $S \subseteq V$ of vertices has a distinct cost. Fix some $1 \leq i \leq r$. Let $\Delta_{i}$ be the unique minimum-cost separator for $\left(s_{i}, t_{i}\right)$. Consider the connected components of $G \backslash \Delta_{i}$ that contain $s_{i}$ and $t_{i}$. Let $S_{i}$ be the component of the smaller cost. We then set $T_{i}=V \backslash\left(S_{i} \cup \Delta_{i}\right)$. This finishes the definition of the cuts $\left(S_{i}, \Delta_{i}, T_{i}\right)$. Clearly, these cuts satisfy property 1 .

We claim that sets $\left\{S_{i}\right\}_{i=1}^{r}$ form a laminar family. Assume for contradiction that this is not the case, so there are two sets $S_{i}, S_{j}$ whose intersection is nonempty, but neither of them is a subset of the other. Assume without loss of generality that these two sets are $S_{1}$ and $S_{2}$. Notice that $c\left(\Delta_{1}\right) \neq c\left(\Delta_{2}\right)$ must hold: otherwise, if $c\left(\Delta_{1}\right)=c\left(\Delta_{2}\right)$, then $\Delta_{1}=\Delta_{2}$ must hold, and thus $S_{1}$ and $S_{2}$ are some connected components of $G \backslash \Delta_{1}$. But in that case, either $S_{1}=S_{2}$ or $S_{1} \cap S_{2}=\varnothing$ must hold, a contradiction. Without loss of generality, we assume that $c\left(\Delta_{1}\right)>c\left(\Delta_{2}\right)$. Since $\Delta_{1}$ is the minimum cost separator for $s_{1}$ and $t_{1}$, and $c\left(\Delta_{2}\right)<c\left(\Delta_{1}\right)$, set $\Delta_{2}$ cannot be a separator for $s_{1}$ and $t_{1}$, and so both of these vertices must either belong to $S_{2}$ or to $T_{2}$.

Let $X_{2} \in\left\{S_{2}, T_{2}\right\}$ be the set that contains neither $s_{1}$ nor $t_{1}$, and let $Y_{2}$ be the other set (recall that by our assumption sets $\Delta_{1}$ and $\Delta_{2}$ contain no terminals). Recall that $X_{2}$ contains either $s_{2}$ or $t_{2}$ but not both of them. Let us assume without loss of generality that $s_{2} \in X_{2}$ and $t_{2} \in Y_{2}$. Let $X_{1} \in\left\{S_{1}, T_{1}\right\}$ be set containing $s_{2}$, and let $Y_{1}$ be the other set. Assume without loss of generality that $s_{1} \in S_{1}$ and $t_{1} \in T_{1}$. Figure 3 shows which terminals lie in which sets. 


\begin{tabular}{c|c|c|c|} 
& $X_{2}$ & $\Delta_{2}$ & $Y_{2}$ \\
\hline$X_{1}$ & $s_{2}$ & & $s_{1},\left(t_{2}\right)$ \\
\hline$\Delta_{1}$ & & & \\
\hline$Y_{1}$ & & & $t_{1},\left(t_{2}\right)$ \\
\hline
\end{tabular}

Fig. 3. The figure shows the intersections of the sets $S_{1}, \Delta_{1}, T_{1}$ with the sets $S_{2}, \Delta_{2}, T_{2}$. There are edges only between sets located in horizontally, vertically, or diagonally adjacent cells. The figure also shows how terminals $s_{1}, t_{1}, s_{2}$, and $t_{2}$ are distributed among the sets, with the two possible locations of $t_{2}$ appearing in parentheses.

We need the following claim.

CLAIM 5.1.

$$
Y_{1} \cap \Delta_{2}=X_{2} \cap \Delta_{1}=\varnothing
$$

Proof. Let $A=\left(Y_{1}, \cap \Delta_{2}\right) \cup\left(Y_{2} \cap \Delta_{1}\right) \cup\left(\Delta_{1} \cap \Delta_{2}\right)$, and let $B=\left(X_{1} \cap \Delta_{2}\right) \cup\left(X_{2} \cap\right.$ $\left.\Delta_{1}\right) \cup\left(\Delta_{1} \cap \Delta_{2}\right)$ (Figure 4). Notice that $A$ is a separator for $s_{1}$ and $t_{1}$, and $B$ is a separator for $s_{2}$ and $t_{2}$.

By our definition of cuts $\left(S_{i}, \Delta_{i}, T_{i}\right)$ :

-either $c\left(\Delta_{1}\right)<c(A)$ or $\Delta_{1}=A$; and

-either $c\left(\Delta_{2}\right)<c(B)$ or $\Delta_{2}=B$.

However, $c(A)+c(B)=c\left(\Delta_{1}\right)+c\left(\Delta_{2}\right)$. Therefore, $\Delta_{1}=A$ and $\Delta_{2}=B$ must hold, and so $Y_{1} \cap \Delta_{2}=X_{2} \cap \Delta_{1}=\varnothing$.

\begin{tabular}{c|c|c|c} 
& $X_{2}$ & $\Delta_{2}$ & $Y_{2}$ \\
\hline$X_{1}$ & \multicolumn{3}{|c}{$\mathbf{X}_{\mathbf{1}}$} \\
\hline$\Delta_{1}$ & $\varnothing$ & \multicolumn{2}{|c}{$\Delta_{1}$} \\
\hline$Y_{1}$ & $\mathbf{Y}_{\mathbf{1}} \cap \mathbf{X}_{\mathbf{2}}$ & $\varnothing$ & $\mathbf{Y}_{\mathbf{1}} \cap \mathbf{Y}_{\mathbf{2}}$
\end{tabular}

Thus, sets $Y_{1} \cap X_{2}, Y_{1} \cap Y_{2}$ and $X_{1}$ are all disconnected in $G \backslash \Delta_{1}$. In other words, $\Delta_{1}$ is a separator for each pair of these three sets. We claim that $S_{1}=X_{1}$ must hold. Indeed, since neither $s_{1}$ nor $t_{1}$ lie in $Y_{1} \cap X_{2}, S_{1} \neq Y_{1} \cap X_{2}$. It is also impossible that $S_{1}=Y_{1} \cap Y_{2}$, as then either $S_{1} \subseteq S_{2}$, or $S_{1} \cap S_{2}=\emptyset$, contradicting our initial assumption.

Similarly, sets $Y_{1} \cap X_{2}, X_{1} \cap X_{2}$, and $Y_{2}$ are disconnected in $G \backslash \Delta_{2}$.

\begin{tabular}{c|c|c|c} 
& $X_{2}$ & $\Delta_{2}$ & $Y_{2}$ \\
\hline$X_{1}$ & $\mathbf{X}_{\mathbf{1}} \cap \mathbf{X}_{\mathbf{2}}$ & \multirow{2}{*}{$\Delta_{2}$} & \multirow{2}{*}{$\mathbf{Y}_{\mathbf{2}}$} \\
\hline$\Delta_{1}$ & $\varnothing$ & & \\
\hline$Y_{1}$ & $\mathbf{Y}_{\mathbf{1}} \cap \mathbf{X}_{\mathbf{2}}$ & $\varnothing$ &
\end{tabular}

We claim that $S_{2}=Y_{2}$ must hold. Indeed, $Y_{1} \cap X_{2}$ does not contain $s_{2}$ or $t_{2}$, so $S_{2} \neq Y_{1} \cap X_{2}$. It is also impossible that $S_{2}=X_{1} \cap X_{2}$, as then $S_{2} \subseteq X_{1} \subseteq S_{1}$ must hold, contradicting our initial assumption. To summarize, we have shown that $S_{1}=X_{1}$, and $S_{2}=Y_{2}$ must hold. But then, by the definition of the sets $S_{i}, c\left(Y_{1} \cap Y_{2}\right)>c\left(S_{1}\right)=c\left(X_{1}\right)$, and $c\left(X_{1} \cap X_{2}\right)>c\left(S_{2}\right)=c\left(Y_{2}\right)$. Therefore, $c\left(Y_{1} \cap Y_{2}\right)+c\left(X_{1} \cap X_{2}\right)>c\left(X_{1}\right)+c\left(Y_{2}\right)$, which is impossible.

Finally, we show that we can perturb the costs of the vertices so that all subsets have different costs. Let

$$
\delta=\min _{\substack{A, B \subset V: \\ c(A)-c(B)>0}} c(A)-c(B)
$$




\begin{tabular}{c|c|c|c|} 
& $X_{2}$ & $\Delta_{2}$ & $Y_{2}$ \\
\hline$X_{1}$ & $s_{2}$ & & $s_{1},\left(t_{2}\right)$ \\
\hline$\Delta_{1}$ & & & \\
\hline$Y_{1}$ & & & $t_{1},\left(t_{2}\right)$ \\
\hline
\end{tabular}

Set $A$

\begin{tabular}{c|c|c|c} 
& $X_{2}$ & $\Delta_{2}$ & $Y_{2}$ \\
\hline$X_{1}$ & $s_{2}$ & & $s_{1},\left(t_{2}\right)$ \\
\hline$\Delta_{1}$ & & & \\
\hline$Y_{1}$ & & & $t_{1},\left(t_{2}\right)$ \\
\hline
\end{tabular}

Set $B$

\begin{tabular}{c|c|c|c} 
& $X_{2}$ & $\Delta_{2}$ & $Y_{2}$ \\
\hline$X_{1}$ & $s_{2}$ & & $s_{1},\left(t_{2}\right)$ \\
\hline$\Delta_{1}$ & $\emptyset$ & & \\
\hline$Y_{1}$ & & $\emptyset$ & $t_{1},\left(t_{2}\right)$ \\
\hline
\end{tabular}

Sets $Y_{1} \cap \Delta_{2}$ and $X_{2} \cap \Delta_{1}$

Fig. 4. Illustration for Claim 5.1.

We assign a new cost $\tilde{c}_{u}$ to every vertex $u$ uniformly at random from the interval $\left[c_{u}, c_{u}+\delta /(2|V|)\right]$. Note that with probability 1 , the costs of every two distinct vertex subsets will be different. We find a family of vertex cuts $\left(S_{i}, \Delta_{i}, T_{i}\right)$ with respect to the $\operatorname{costs} \tilde{c}_{v}$. We verify that $\Delta_{i}$ is a minimum cost separator for $s_{i}$ and $t_{i}$ with respect to the original costs $c_{v}$. Assume by contradiction that there is a separator $\Delta$ for $s_{i}$ and $t_{i}$, with $c(\Delta)<c\left(\Delta_{i}\right)$.

Then

$$
\tilde{c}\left(\Delta_{i}\right) \geq c\left(\Delta_{i}\right) \geq c(\Delta)+\delta>c(\Delta)+\frac{\delta}{2|V|} \cdot|\Delta| \geq \tilde{c}(\Delta),
$$

which contradicts to the fact that $\Delta_{i}$ is the minimum cost separator for $s_{i}$ and $t_{i}$ with respect to costs $\tilde{c}$.

\section{Proof of Theorem 1.3}

In this section, we prove Theorem 1.3 by showing a $\left(2, O\left(d k \log ^{5 / 2} r \log \log r\right)\right)$ bi-criteria approximation algorithm VC-kRC, where $d$ is the maximum number of demand pairs in which any terminal participates. The running time of the algorithm is $n^{O(k)}$.

We start by extending the definition of the $k$-route sparsest cut to the vertex connectivity version. Given two disjoint subsets $S, T$ of vertices, let $D(S, T)$ be the set of all demand pairs $\left(s_{i}, t_{i}\right)$ with exactly one of the vertices $s_{i}, t_{i}$ lying in $S$ and the other one lying in $T$. Given any pair $(S, \Delta)$ of disjoint subsets, let $\Upsilon^{(\Delta)}(S)=\sum_{e \in E(S, V \backslash(S \cup \Delta))} w_{e}$, where $E(S, V \backslash(S \cup \Delta))$ is the subset of all edges with one endpoint in $S$ and the other endpoint in $V \backslash(S \cup \Delta)$.

The $k$-route vertex sparsity of the set $S$ is then defined to be

$$
\tilde{\Psi}^{(k)}(S)=\min _{\substack{\Delta \subseteq V \backslash S: \\|\Delta| \leq k-1}}\left\{\frac{\Upsilon^{(\Delta)}(S)}{|D(S, V \backslash(S \cup \Delta))|}\right\},
$$

and the $k$-route vertex sparsity of the graph $G$ is

$$
\tilde{\Psi}^{(k)}(G)=\min _{S \subset V}\left\{\tilde{\Psi}^{(k)}(S)\right\}
$$

It is easy to see that similarly to the edge version of $k$-route sparsest cut, the $k$-route vertex sparsest cut can be approximated in time $n^{O(k)}$ to within a factor of $\alpha_{\mathrm{ALN}}(r)$, as we show in the next theorem.

THEOREM 5.2. There is an algorithm that finds, in time $n^{O(k)}$, disjoint subsets $S, \Delta \subset V$ of vertices, with $|\Delta| \leq k-1$ such that

$$
\Upsilon^{(\Delta)}(S) \leq \alpha_{\mathrm{ALN}}(r) \cdot \tilde{\Psi}^{(k)}(G) \cdot|D(S, V \backslash(S \cup \Delta))| .
$$

Proof. For every subset $\Delta \subset V$ of at most $k-1$ vertices, we use the algorithm $\mathcal{A}_{\mathrm{ALN}}$ to find an approximate sparsest cut in the graph $G \backslash \Delta$ and output the sparsest cut among all such cuts. 
Input: A weighted graph $G=(V, E)$ with a set $D=\left\{\left(s_{i}, t_{i}\right)\right\}_{1 \leq i \leq r}$ of demand pairs, and edge weights $\left\{w_{e}\right\}_{e \in E}$.

Output: A subset $E^{\prime}$ of edges such that no demand pair $s_{i}$ and $t_{i}$ is $(2 k-1)$-vertex connected in $G \backslash E^{\prime}$.

(1) If $r=0$ return $E^{\prime}=\emptyset$.

(2) Find sets $U$ and $\Delta$ with $|\Delta| \leq 2 k-1$ and $\Upsilon^{(\Delta)}(U) \leq \alpha_{\mathrm{ALN}}(r) \cdot \tilde{\Psi}^{(2 k-1)}(G) \cdot \mid D(U, V \mid$ $(U \cup \Delta)) \mid$ using Theorem 5.2.

(3) Let $E_{0}=E(U, V \backslash(U \cup \Delta))$, and let $G^{\prime}=G \backslash E_{0}$.

(4) Let $D_{0}$ be the set of all demand pairs $\left(s_{i}, t_{i}\right)$ that are no longer $(2 k-1)$-vertex connected in $G^{\prime}$.

(5) Solve the problem recursively on $G^{\prime}$ with the set $D \backslash D_{0}$ of demand pairs to obtain a solution $E_{1}$.

(6) Return $E^{\prime}=E_{0} \cup E_{1}$.

Fig. 5. Bi-criteria approximation algorithm for VC-kRC in time $n^{O(k)}$.

Our algorithm for VC-kRC is very similar to the algorithm for EC-kRC from Section 4. The only difference is that we use Theorem 5.2 to find an approximate $k$-route vertex sparsest cut. The algorithm is summarized in Figure 5.

It is easy to verify that if $E^{\prime}$ is the solution computed by the algorithm, then for each demand pair $\left(s_{i}, t_{i}\right)$ there are at most $(2 k-1)$ vertex-disjoint paths connecting them in $G \backslash E^{\prime}$. This is because the algorithm only removes a demand pair $\left(s_{i}, t_{i}\right)$ when the terminals $s_{i}$ and $t_{i}$ are no longer $(2 k-1)$-vertex connected, and it terminates, as it removes at least one demand pair in each iteration.

To analyze the performance of the algorithm, we use the following theorem, which relates the value $\tilde{\Psi}^{(k)}(G)$ of the $k$-route vertex sparsest cut in graph $G$ to the value OPT of the optimal solution to VC-kRC.

THEOREM 5.3.

$$
\tilde{\Psi}^{(2 k-1)}(G) \leq O\left(\frac{d k \log r}{r}\right) \cdot \text { OPT. }
$$

Proof. Let $H=G \backslash E^{*}$. The proof roughly follows the proof of Theorem 3.1, except that we need one additional step, which is summarized in the following lemma.

Lemma 5.4. There exists a subset $D^{\prime} \subseteq D$ of $r^{\prime}=\Omega(r /(d k))$ demand pairs, and a collection of vertex cuts $\left\{\left(S_{i}, \Delta_{i}, T_{i}\right)\right\}_{\left(s_{i}, t_{i}\right) \in D^{\prime}}$, such that

-For all $\left(s_{i}, t_{i}\right) \in D^{\prime}, \Delta_{i}$ is a separator for $\left(s_{i}, t_{i}\right)$ in $H,\left|\Delta_{i}\right|<k$, and $\Delta_{i} \cap T^{\prime}=\emptyset$, where $T^{\prime}$ is the set of all terminals participating in demand pairs in $D^{\prime}$. $-\left\{S_{i}\right\}_{\left(s_{i}, t_{i}\right) \in D^{\prime}}$ is a laminar family of vertex subsets.

Proof. For each $1 \leq i \leq r$, let $\Delta_{i}^{\prime}$ be a minimum vertex separator for $s_{i}$ and $t_{i}$ in $H$. Since $s_{i}$ and $t_{i}$ are not $k$-vertex connected in $H,\left|\Delta_{i}^{\prime}\right|<k$. We construct an auxiliary graph $Z$, whose vertex set is $[r]$ - that is, each vertex $i$ of $Z$ represents the demand pair $\left(s_{i}, t_{i}\right)$. We say that a demand $i$ blocks another demand $j$ if and only if $\Delta_{i}^{\prime}$ contains either $s_{j}$ or $t_{j}$ (or both). We connect $i$ and $j$ with an edge in $Z$ if and only if one of them blocks the other. Since $\left|\Delta_{i}^{\prime}\right| \leq k-1$ and each vertex in $\Delta_{i}^{\prime}$ participates in at most $d$ demand pairs, demand $i$ blocks at most $d(k-1)$ demands. Therefore, there are at most $d(k-1) r$ edges in $Z$. By Turan's theorem, there is an independent set $I$ of size $\Omega(r /(d k))$ in $Z$. Let $r^{\prime}=|I|$, and let $D^{\prime}=\left\{\left(s_{i}, t_{i}\right) \mid i \in I\right\}$.

Next, we apply Lemma 5.1 to graph $G$ with the set $D^{\prime}$ of demand pairs, where we define the cost $c_{u}$ of every vertex $u$ as follows: $c_{u}=|V|$ if $u=s_{i}$ or $u=t_{i}$ for some 
$\left(s_{i}, t_{i}\right) \in D^{\prime}$, and $c_{u}=1$ otherwise. Since demand pairs in $D^{\prime}$ do not block each other, the minimum cost vertex cut for each of them has cost at most $k-1<|V|$. Let $\left\{\left(S_{i}, \Delta_{i}, T_{i}\right)\right\}_{\left(s_{i}, t_{i}\right) \in D^{\prime}}$ be the collection of cuts returned by Lemma 5.1 . It is easy to see that these cuts satisfy the conditions of the lemma.

We use Lemma 5.4 to find a subset $D^{\prime}$ of demand pairs and vertex cuts $\left(S_{i}, \Delta_{i}, T_{i}\right)$. We assume without loss of generality that $D^{\prime}=\left\{\left(s_{1}, t_{1}\right), \ldots,\left(s_{r^{\prime}}, t_{r^{\prime}}\right)\right\}$. Now we need the following counterpart of Lemma 4.2.

Lemma 5.5. There is a family $\mathcal{P}=\left\{U_{1}, \ldots, U_{p}\right\}$ of disjoint vertex subsets, and a collection $\left\{\left(U_{j}, \Lambda_{j}, R_{j}\right)\right\}_{j=1}^{p}$ of vertex cuts in graph $H$, such that

- for each $1 \leq j \leq p,\left|\Lambda_{j}\right|<2 k-1$,

$-\sum_{j=1}^{p}\left|D\left(U_{j}, R_{j}\right)\right| \geq \frac{r^{\prime}}{8 \log r^{\prime}}$.

Proof. The proof closely follows the proof of Lemma 4.2. Let $\mathcal{S}=\left\{S_{1}, \ldots, S_{r^{\prime}}\right\}$ be the family of vertex subsets from Lemma 5.4, and assume that the vertex cut corresponding to set $S_{i} \in \mathcal{S}$ is $\left(S_{i}, \Delta_{i}, T_{i}\right)$. Family $\mathcal{P}$ will contain two types of vertex subsets. Subset $U_{j}$ is a subset of the first type if and only if $U_{j}=S_{i}$ for some $S_{i} \in \mathcal{S}$. In this case, we set $\Lambda_{j}=\Delta_{i}$, and the corresponding cut $\left(U_{j}, \Lambda_{j}, R_{j}\right)=\left(S_{i}, \Delta_{i}, T_{i}\right)$. It is easy to see that the first condition of the lemma will hold for vertex subsets of this type.

Subset $U_{j}$ of vertices is a subset of the second type if and only if $U_{j}=S_{i} \backslash\left(S_{i^{\prime}} \cup \Delta_{i^{\prime}}\right)$ for some $S_{i}, S_{i^{\prime}} \in \mathcal{S}$, where $S_{i^{\prime}} \subset S_{i}$. In this case, we set $\Lambda_{j}=\Delta_{i} \cup \Delta_{i^{\prime}}$, and $R_{j}=V \backslash\left(U_{j} \cup \Lambda_{j}\right)$. Notice that if $e=(u, v) \in E(H)$ has $u \in U_{j}, v \notin U_{j}$, then $v \in \Lambda_{j}$ must hold. Indeed, if $v \notin S_{i}$, then since $\left(S_{i}, \Delta_{i}, T_{i}\right)$ is a valid vertex cut, $v \in \Delta_{i}$ must hold. Otherwise, if $v \in S_{i}$ but $v \notin \Delta_{i^{\prime}}$, then $v \in S_{i^{\prime}}$ must hold, and since $\left(S_{i^{\prime}}, \Delta_{i^{\prime}}, T_{i^{\prime}}\right)$ is a valid vertex cut, $u \in \Delta_{i^{\prime}}$ must hold, which is impossible. Therefore, $\left(U_{j}, \Lambda_{j}, R_{j}\right)$ is a valid vertex cut. Moreover, $\left|\Lambda_{j}\right|=\left|\Delta_{i} \cup \Delta_{i^{\prime}}\right| \leq 2(k-1)$, and so the first condition of the lemma holds.

We now show how to define the family $\mathcal{P}$ so that the second condition of the lemma is satisfied as well. We assume without loss of generality that $\mathcal{S}$ does not contain two copies of the same set: otherwise, we simply remove copies of the same set until just one copy remains in $\mathcal{S}$.

For every set $S_{i} \in \mathcal{S}$, let $D^{\prime}\left(S_{i}\right)=\bigcup_{\substack{S_{j} \in \mathcal{S} \\ S_{j} \subset S_{i}}} D\left(S_{j}, T_{j}\right) \cap D^{\prime}$, and let $q\left(S_{i}\right)=\mid\left(D\left(S_{i}, T_{i}\right) \cap\right.$ $\left.D^{\prime}\right) \backslash D^{\prime}\left(S_{i}\right) \mid$. As before, we partition the set $\mathcal{S}$ as follows: for $x=1, \ldots,\left\lfloor\log _{2} r^{\prime}\right\rfloor+1$, let $\mathcal{S}_{x}=\left\{S_{i} \in \mathcal{S} \mid 2^{x-1} \leq q\left(S_{i}\right)<2^{x}\right\}$.

Since $\sum_{x=1}^{\left\lfloor\log _{2} r^{\prime}\right\rfloor+1} \sum_{S_{i} \in \mathcal{S}_{x}} q\left(S_{i}\right)=r^{\prime}$, we can choose an index $x^{*}$ such that $\sum_{S_{i} \in \mathcal{S}_{x^{*}}} q\left(S_{i}\right) \geq$ $\frac{r^{\prime}}{2 \log r^{\prime}}$. We say that a set $S_{i}$ is good if it belongs to $\mathcal{S}_{x^{*}}$. Consider the decomposition forest $\mathcal{F}$ for the good sets $S_{i}$ : the nodes of the forest are the sets of $\mathcal{S}_{x^{*}}$, and $S_{i}$ is the parent of $S_{j}$ if and only if $S_{j} \subset S_{i}$, and there is no other set $S_{\ell} \in \mathcal{S}_{x^{*}}$ with $S_{j} \subset S_{\ell} \subset S_{i}$. Let $\mathcal{S}^{\prime}$ be the subset of nodes of $\mathcal{F}$ with at most one child. Note that

$$
\left|\mathcal{S}^{\prime}\right| \geq\left|\mathcal{S}_{x^{*}}^{\prime}\right| / 2 \geq \frac{\sum_{S_{i} \in \mathcal{S}_{x^{*}}^{\prime}} q\left(S_{i}\right)}{2 \cdot 2^{x^{*}}} \geq \frac{r^{\prime}}{2^{x^{*}+1}\left(2 \log r^{\prime}\right)} .
$$

On the other hand, since $q\left(S_{i}\right) \geq 2^{x^{*}-1}$ for $S_{i} \in \mathcal{S}^{\prime}$,

$$
\sum_{S_{i} \in \mathcal{S}^{\prime}} q\left(S_{i}\right) \geq \frac{r^{\prime}}{8 \log r^{\prime}} .
$$

For every set $S_{i} \in \mathcal{S}^{\prime}$, we let $U_{i}=S_{i}$ and $\Lambda_{i}=\Delta_{i}$ if $S_{i}$ is a leaf of $\mathcal{F}$; we let $U_{i}=$ $S_{i} \backslash\left(S_{j} \cup \Delta_{j}\right)$ and $\Lambda_{i}=\Delta_{i} \cup \Delta_{j}$ if $S_{i}$ has a unique child $S_{j}$ in $\mathcal{F}$. Let $R_{i}=V \backslash\left(U_{i} \cup \Lambda_{i}\right)$. 
If set $U_{i}$ is of the first type, then $D\left(U_{i}, R_{i}\right)=D\left(S_{i}, T_{i}\right)$, and so $\left|D\left(U_{i}, R_{i}\right) \cap D^{\prime}\right| \geq q\left(S_{i}\right)$. Otherwise, if $U_{i}=S_{i} \backslash\left(S_{j} \cup \Delta_{j}\right)$, then $D\left(U_{i}, R_{i}\right) \cap D^{\prime}$ contains all demand pairs in $D\left(S_{i}, T_{i}\right) \cap D^{\prime}$, except for pairs $(x, y)$ with $x \in S_{j} \cup \Delta_{j}, y \in T_{i}$. But since $\Delta_{j}$ does not contain any terminals participating in pairs in $D^{\prime}, x \in S_{j}, y \in T_{j}$ and $(x, y) \in D\left(S_{j}, T_{j}\right)$ must hold. Therefore, $\left|D\left(U_{i}, R_{i}\right) \cap D^{\prime}\right| \geq q\left(S_{i}\right)$, and so $\sum_{U_{i} \in \mathcal{P}}\left|D\left(U_{i}, R_{i}\right)\right| \geq \frac{r^{\prime}}{8 \log r^{\prime}}$.

Consider the family $\mathcal{P}=\left\{U_{1}, \ldots, U_{p}\right\}$ and the corresponding cuts $\left(U_{i}, \Lambda_{i}, R_{i}\right)$ as in Lemma 5.5. Since all sets in $\mathcal{P}$ are mutually disjoint, and for each such set $U_{i} \in \mathcal{P}$, $\left|\Lambda_{i}\right| \leq 2 k-1$,

$$
\sum_{j=1}^{p} \Upsilon^{\left(\Lambda_{j}\right)}\left(U_{j}\right) \leq 2 \mathrm{OPT}
$$

and so

$$
\begin{aligned}
\frac{\sum_{j=1}^{p} \Upsilon^{\left(\Lambda_{j}\right)}\left(U_{j}\right)}{\sum_{j=1}^{p}\left|D\left(U_{j}, R_{j}\right)\right|} & \leq O\left(\frac{\log r^{\prime}}{r^{\prime}}\right) \cdot \text { OPT } \\
& \leq O\left(\frac{d k \log r}{r}\right) \cdot \text { OPT }
\end{aligned}
$$

Therefore, there is an index $1 \leq j \leq p$ such that

$$
\frac{\Upsilon^{\left(\Lambda_{j}\right)}\left(U_{j}\right)}{\left|D\left(U_{j}, R_{j}\right)\right|} \leq O\left(\frac{d k \log r}{r}\right) \cdot \text { OPT. }
$$

The left-hand side of this inequality is at least $\tilde{\Psi}^{(2 k-1)}(G)$ since $\left|\Lambda_{j}\right| \leq 2 k-2$. We conclude that $\tilde{\Psi}^{(2 k-1)}(G) \leq O\left(\frac{d k \log r}{r}\right)$. OPT.

To complete the proof of Theorem 1.3, observe that $w\left(E_{0}\right)=\Upsilon^{(\Delta)}(U)$, and by Theorem 5.3,

$$
\begin{aligned}
w\left(E_{0}\right) & \leq \alpha_{\mathrm{ALN}}(r) \tilde{\Psi}^{(2 k-1)}(G)|D(U, V \backslash(U \cup \Delta))| \\
& \leq O\left(\frac{d k \log r}{r}\right) \cdot \alpha_{\mathrm{ALN}}(r) \cdot \mathrm{OPT} \cdot|D(U, V \backslash(U \cup \Delta))| .
\end{aligned}
$$

Note that we remove all demand pairs in $D(U, V \backslash(U \cup \Delta))$ in step 4 of the algorithm. We can now use Theorem 2.5 with $\alpha=O\left(d k \log r \cdot \alpha_{\mathrm{ALN}}(r)\right)$ to conclude that the cost of the solution returned by the algorithm is bounded by $O\left(d k \log ^{5 / 2} r \log \log r\right)$. OPT.

\section{ALGORITHMS FOR 2-ROUTE CUTS}

In this section, we prove Theorem 1.5. Since we prove in Appendix A that EC-kRC can be cast as a special case of VC-kRC, and the connectivity value $k$ remains unchanged in this reduction, it is enough to prove the theorem for VC-kRC, where $k=2$. In the rest of this section, we show an efficient $O\left(\log ^{3 / 2} r\right)$-approximation algorithm for VC-kRC with $k=2$.

Given a subset $S$ of vertices in graph $G$, the uniform vertex 2-route sparsity of $S$ is

$$
\Psi^{(2)}(S)=\min _{\substack{\Delta \subseteq V \backslash S: \\|\Delta| \leq 1}}\left\{\frac{\Upsilon^{(\Delta)}(S)}{\min \{D(S), D(V \backslash(S \cup \Delta))\}}\right\},
$$

and the uniform vertex 2-route sparsity of the graph $G$ is

$$
\Psi^{(2)}(G)=\min _{S \subset V}\left\{\Psi^{(2)}(S)\right\}
$$


Input: A weighted graph $G=(V, E)$ with demand pairs $\left\{\left(s_{i}, t_{i}\right)\right\}_{1<i<r}$, and edge weights $\left\{w_{e}\right\}_{e \in E}$, such that each demand pair has at least 2 vertex-disjoint paths connecting them in $G$.

Output: A set $E^{\prime}$ of edges such that each pair $\left(s_{i}, t_{i}\right)$ is no longer 2-vertex connected in $G \backslash E^{\prime}$.

(1) If $r=0$ return $E^{\prime}=\emptyset$.

(2) Find disjoint subsets $S, \Delta$ of vertices with $|\Delta|=1,0<D(S) \leq r$, such that $\Upsilon^{(\Delta)}(S) \leq \alpha_{\mathrm{ARV}}(r) \cdot \Psi^{(2)}(G) \cdot D(S)$, using Theorem 6.1. Let $T=V \backslash(S \cup \Delta)$.

(3) Let $E_{0}=E(S, T) ; G^{\prime}=G \backslash E_{0}$.

(4) Remove all demand pairs $\left(s_{i}, t_{i}\right)$ that are no longer 2-vertex connected in $G^{\prime}$.

(5) Recursively solve the subinstances induced by $G[S \cup \Delta]$ and $G[T \cup \Delta]$ to obtain solutions $E_{1}$ and $E_{2}$. The set of demand pairs for the instance induced by $G[S \cup \Delta]$ is defined to be the subset of all remaining demand pairs contained in $S \cup \Delta$. The set of demand pairs for the instance induced by $G[T \cup \Delta]$ is defined similarly.

(6) Return $E^{\prime}=E_{0} \cup E_{1} \cup E_{2}$.

Fig. 6. Approximation algorithm for VC-kRC, $k=2$ (weighted case).

As before, we can efficiently approximate the uniform vertex 2-route sparsest cut in any graph, as shown in the next theorem.

TheOREm 6.1. There is a polynomial time algorithm that finds disjoint subsets $S \subset V$ and $\Delta \subset V$ of vertices, with $|\Delta| \leq 1$ and $0<D(S) \leq r$, such that

$$
\Upsilon^{(\Delta)}(S) \leq \alpha_{\mathrm{ARV}}(r) \cdot \Psi^{(2)}(G) \cdot D(S) .
$$

Proof. For every subset $\Delta \subset V$ of size at most 1 , we use the algorithm $\mathcal{A}_{\mathrm{ARV}}$ to find the $\alpha_{\mathrm{ARV}}(r)$-approximate uniform sparsest cut in graph $G \backslash \Delta$ and output the cut with the smallest sparsity.

The approximation algorithm for VC-kRC with $k=2$ is shown in Figure 6.

To analyze the algorithm, we start by showing that it is guaranteed to produce a feasible solution.

Claim 6.1. The algorithm outputs a feasible solution to the problem.

Proof. The proof is by induction on the number of vertices in $G$. Assume that the algorithm outputs a feasible solution for all graphs containing fewer than $n$ vertices, and consider a graph $G$ containing $n$ vertices. Let $\left(s_{i}, t_{i}\right)$ be any demand pair, and assume for contradiction that there are at least two vertex-disjoint simple paths $P_{1}, P_{2}$ connecting $s_{i}$ to $t_{i}$ in $G \backslash E^{\prime}$. Observe first that either $s_{i}, t_{i} \in S \cup \Delta$ or $s_{i}, t_{i} \in T \cup \Delta$ must hold. Otherwise, one of the two vertices must belong to $S$ and the other to $T$. But $\Delta$ is a separator for $S$ and $T$ in graph $G^{\prime}$, and since $|\Delta|=1$, the paths $P_{1}$ and $P_{2}$ cannot be vertex disjoint. Assume without loss of generality that $s_{i}, t_{i} \in S \cup \Delta$. By the induction hypothesis, $E_{1}$ is a feasible solution to the instance induced by $G[S \cup \Delta]$, and in particular, $G[S \cup \Delta] \backslash E_{1}$ cannot contain two vertex-disjoint paths connecting $s_{i}$ to $t_{i}$. Therefore, at least one of the two paths, say $P_{1}$, must contain a vertex of $T$. But since $\Delta$ is a separator for $S$ and $T,|\Delta|=1$, and both $s_{i}, t_{i} \notin T$, path $P_{1}$ cannot be a simple path, a contradiction.

It now remains to bound the cost of the solution produced by the algorithm. As before, we do so by relating the value of the 2-route vertex sparsest cut to the value OPT of the optimal solution to the VC-kRC problem. 
THEOREM 6.2. Suppose that we are given an undirected graph $G=(V, E)$ with edge weights $w_{e}$ and $r$ demand pairs $\left(s_{1}, t_{1}\right), \ldots,\left(s_{r}, t_{r}\right)$. Let OPT be the cost of the optimal solution to the corresponding VC-kRC problem instance, and assume that $k=2$. Then

$$
\Psi^{(2)}(G) \leq \frac{4 \mathrm{OPT}}{r} .
$$

Proof. We will assume that $G$ is connected: if $G$ is not connected and all terminals lie in one connected component, then we just let $G$ be this connected component; otherwise, if some terminals lie in one connected component and others lie in another connected component, then $\Psi^{(2)}(G)=0$ and we are done. Consider the graph $H=G \backslash E^{*}$. By the optimality of $E^{*}$, graph $H$ is connected. Since $E^{*}$ is a solution to the VC-kRC problem with $k=2$, for every demand pair $\left(s_{i}, t_{i}\right)$, there is a one-vertex subset $\Delta_{i}$ of vertices separating $s_{i}$ from $t_{i}$ in $H$.

Consider the block decomposition of $H$. Recall that a block $B$ of $H$ is a maximal 2 -vertex connected subgraph of $H$. Every pair $\left(B_{1}, B_{2}\right)$ of distinct blocks share either no vertices or only one vertex, and in the latter case, this vertex is a cut vertex. Let $\mathcal{B}$ be the set of all blocks, and let $S$ be the set of all cut vertices of $H$. The block tree $B(H)$ of $H$ is a bipartite graph, with parts $\mathcal{B}$ and $S$, in which a block $B$ is connected to a cut vertex $u$ if and only if $u$ lies in $B$. We assign costs $c(u)$ to each node $u$ of the tree $B(H)$ as follows. If $u$ is a cut vertex of $H$, let $c(u)=D_{u}$ (the number of demand pairs in which $u$ participates); if $B$ is a block, then $c(B)=D(V(B) \backslash S)$. Then each vertex $u \in S$ contributes $D_{u}$ to the cost of $u$ in $B(H)$; each vertex $u \in V \backslash S$ contributes $D_{u}$ to the cost of the block that contains $u$. Thus, the total cost of all vertices in $B(H)$ is exactly $2 r$. Note that the cost $c(u)$ of a cut vertex $u$ is at most $r$ (since there are $r$ demand pairs); the cost $c(u)$ of a block $B$ is also at most $r$ since for every $i \in[r]$, the block $B$ contains at most one of the terminals $s_{i}$ and $t_{i}$.

Assume first that we can find a block $B$ in the tree $B(H)$ such that if $u_{1}, \ldots, u_{p}$ are the neighbors of $B$ in the tree $B(H)$ (recall that they must be the cut vertices of $H$ that lie in $B$ ), and for each $1 \leq i \leq p, \mathcal{T}_{i}$ is the subtree of $B(H)$ rooted at $u_{i}$, then $c\left(\mathcal{T}_{i}\right) \leq 5 r / 4$.

For each $1 \leq i \leq p$, let $S_{i}$ be the union of all blocks whose corresponding nodes lie in the tree $\mathcal{T}_{i}$, excluding the vertex $u_{i}$-that is, $S_{i}=\left(\bigcup_{B^{\prime} \in \mathcal{T}_{i}} V\left(B^{\prime}\right)\right) \backslash\left\{u_{i}\right\}$-and let $T_{i}=V \backslash\left(S_{i} \cup\left\{u_{i}\right\}\right)$.

Note that $u_{i}$ is a separator vertex for $S_{i}$ and $T_{i}$ in $H$. By our choice of $B, D\left(S_{i}\right) \leq 5 r / 4$, and $D\left(T_{i}\right)=2 r-D\left(S_{i}\right)-D\left(u_{i}\right) \geq 3 r / 4 \geq D\left(S_{i}\right) / 2\left(\right.$ since $\left.D\left(S_{i}\right)+D\left(u_{i}\right)=c\left(\mathcal{T}_{i}\right) \leq 5 r / 4\right)$. On the other hand, $\sum_{i=1}^{p} D\left(S_{i}\right)=2 r-\overline{D(B)} \geq r$.

For each $1 \leq i \leq p$, there are no edges between $S_{i}$ and $V \backslash\left(S_{i} \cup\left\{u_{i}\right\}\right)$ in $H$, and thus we have

$$
\begin{aligned}
w\left(E^{*} \cap E_{G}\left(S_{i}, T_{i}\right)\right) & =w\left(E_{G}\left(S_{i}, T_{i}\right)\right) \\
& \geq \Psi^{(2)}(G) \cdot \min \left\{D\left(S_{i}\right), D\left(T_{i}\right)\right\} \\
& \geq \Psi^{(2)}(G) \cdot D\left(S_{i}\right) / 2 .
\end{aligned}
$$

Summing this inequality over all $1 \leq i \leq p$, we get

$$
\begin{aligned}
2 \mathrm{OPT} & \geq \sum_{i=1}^{p} w\left(E^{*} \cap E_{G}\left(S_{i}, T_{i}\right)\right) \geq \sum_{i=1}^{p} \Psi^{(2)}(G) \cdot D\left(S_{i}\right) / 2 \\
& \geq \Psi^{(2)}(G) \cdot r / 2 .
\end{aligned}
$$

We conclude that $\Psi^{(2)}(G) \leq 4 \mathrm{OPT} / r$.

We find a node $x$ in the tree $B(H)$ with the following property: if we root the tree $B(H)$ at $x$, then for each child $x^{\prime}$ of $x$, the cost of the subtree rooted at $x^{\prime}$ is at most $r$ (half of the cost of $B(H)$ ). To find such a node $x$, start with an arbitrary node $x_{0}=x$ and root 
the tree $B(H)$ at $x$. As long as $x$ has a child $x^{\prime}$, such that the cost of the subtree rooted at $x^{\prime}$ is more than $r$, we set $x=x^{\prime}$ and continue. Since during this process we always move down the tree rooted at $x_{0}$, it is guaranteed to terminate. It is easy to verify that the node $x$ at which the process terminates has the desired properties. If the node $x$ where this process terminates is a block, then block $x$ satisfies the assumption we discussed earlier, and therefore, $\Psi^{(2)}(G) \leq 4 \mathrm{OPT} / r$. Therefore, we assume that $x$ is a cut vertex. Let $\mathcal{T}_{1}, \ldots, \mathcal{T}_{p}$ be the set of the subtrees of $B(H)$ rooted at the children nodes of $x$, sorted by their cost, with $\mathcal{T}_{1}$ being the most expensive subtree and $\mathcal{T}_{p}$ the cheapest one.

Assume first that $c\left(\mathcal{T}_{1}\right) \leq r / 2$. Note that $\sum_{i=1}^{p} c\left(\mathcal{T}_{i}\right)=2 r-c(x) \geq r$. Let $j^{*}$ be the largest index $j$ such that $\sum_{i=1}^{j} c\left(\mathcal{T}_{i}\right) \leq r / 2$. By our choice of $x$, we have $1 \leq j^{*}<p$. Clearly, $\sum_{i=1}^{j^{*}} c\left(\mathcal{T}_{i}\right) \geq \frac{r}{4}$.

Let $S$ be the set of all vertices of $H$ contained in all blocks $B \in V\left(\mathcal{T}_{i}\right)$, for all $1 \leq i \leq j^{*}$ (excluding the vertex $x$ ), and let $T=V \backslash(S \cup\{x\})$. Then $D(T)=2 r-D(S)-D(x) \geq r / 2$. Therefore, $\Psi^{(2)}(G) \leq \Psi^{(2)}(S) \leq \frac{\Upsilon^{((x))}(S)}{\min \{D(S), D(T)\}} \leq \frac{\mathrm{OPT}}{r / 4}$.

Assume now that $c\left(\mathcal{T}_{1}\right)>r / 2$, but $c\left(\mathcal{T}_{1}\right)+c(x) \leq 7 r / 4$. Then $\sum_{j=2}^{p} c\left(\mathcal{T}_{p}\right) \geq r / 4$. Setting $S$ to be the union of all blocks whose vertices lie in the trees $\mathcal{T}_{j}$ for $j \neq 1$ (excluding the vertex $x)$, and setting $T=V \backslash(S \cup\{x\})$, we get that $D(S) \geq r / 4, D(T) \geq r / 2$, and so $\Psi^{(2)}(G) \leq \Psi^{(2)}(S) \leq \frac{\Upsilon^{((x))}(S)}{\min \{D(S), D(T)\}} \leq \frac{\mathrm{OPT}}{r / 4}$ as before.

Finally, if $c\left(\mathcal{T}_{1}\right)+c(x)>7 r / 4$, let $B$ denote the child of $x$ that serves as the root of $\mathcal{T}_{1}$. Note that $B$ has the property that every tree rooted at a neighbor of $B$ has a cost of at most $5 r / 4$ : if the neighbor is in $\mathcal{T}_{1}$ then the subtree lies in $\mathcal{T}_{1}$ and thus its cost is at most $r$ by our choice of $x$; otherwise, if the neighbor is $x$, then the cost of the tree rooted at $x$ is $2 r-c\left(\mathcal{T}_{1}\right)=2 r+c(x)-\left(c\left(\mathcal{T}_{1}\right)+c(x)\right) \leq 2 r+r-7 r / 4=5 r / 4$. As we have shown, $\Psi^{(2)}(G) \leq 4 \mathrm{OPT} / r$ in this case.

Let $a$ be the number of demand pairs contained in $S \cup \Delta$ and $b$ be the number of demand pairs contained in $(V \backslash S) \cup \Delta$ in graph $G^{\prime}$. From Theorem 6.2,

$$
\begin{aligned}
w\left(E_{0}\right) & \leq \alpha_{\mathrm{ARV}}(r) \cdot \Psi^{(2)}(G) \cdot D(S) \\
& \leq 4 \alpha_{\mathrm{ARV}}(r) \text { OPT } \min \{a, b\} / r \\
& \leq 4 \alpha_{\mathrm{ARV}}(r) \text { OPT } \min \{r-a, r-b\} / r .
\end{aligned}
$$

Therefore, by setting $\alpha=4 \alpha_{\mathrm{ARV}}(r)$, we get the same recurrence as in the proof of Theorem 2.4:

$$
w\left(E^{\prime}\right) \leq w\left(E_{1}^{\prime}\right)+w\left(E_{2}^{\prime}\right)+2 \alpha \cdot \frac{\min \{r-a, r-b\} \text { OPT }}{r} .
$$

Solving this recurrence as in Theorem 2.4, we get that $w\left(E^{\prime}\right) \leq O\left(\log ^{3 / 2} r\right)$ OPT.

\section{A FACTOR $\boldsymbol{k}$-HARDNESS FOR $\boldsymbol{k}$-VC-kRC}

In this section, we prove Theorem $1.4 .^{3}$ We perform a reduction from the 3SAT(5) problem. In this problem, we are given a 3SAT formula $\varphi$ on $n$ variables and $5 n / 3$ clauses. Each clause contains three distinct literals, and each variable participates in exactly five different clauses. We say that $\varphi$ is a Yes-Instance if it is satisfiable. We say that $\varphi$ is a No-Instance with respect to some parameter $\epsilon$ if and only if no assignment satisfies more than $\epsilon$-fraction of clauses. The following well-known theorem follows from the PCP theorem [Arora and Safra 1998; Arora et al. 1998].

\footnotetext{
${ }^{3}$ We note that our original proof contained an error, which was pointed out to us by Bundit Laekhanukit (personal communication). We include a corrected proof here.
} 
Theorem 7.1. There is a constant $\epsilon: 0<\epsilon<1$ such that it is NP-hard to distinguish between Yes-Instances and No-Instances (defined with respect to $\epsilon$ ) of the 3SAT(5) problem.

We use the Raz verifier for 3SAT(5) with $\ell$ parallel repetitions. This is an interactive proof system in which two provers try to convince the verifier that the input 3SAT(5) formula $\varphi$ is satisfiable. The verifier chooses, independently at random, $\ell$ clauses $C_{1}, \ldots, C_{\ell}$, and for each $i: 1 \leq i \leq \ell$, a variable $x_{i}$ participating in clause $C_{i}$ is chosen at random. The verifier then sends one query to each one of the two provers, in which the query to the first prover consists of the indices of the variables $x_{1} \ldots, x_{\ell}$, and the query to the second prover contains the indices of the clauses $C_{1}, \ldots, C_{\ell}$. The first prover returns an assignment to variables $x_{1}, \ldots, x_{\ell}$. The second prover is expected to return an assignment to all variables in clauses $C_{1}, \ldots, C_{\ell}$, which must satisfy the clauses. Finally, the verifier checks that the answers of the two provers are consistent-that is, for each $i: 1 \leq i \leq \ell$, the assignment to $x_{i}$, returned by the first prover, is identical to the assignment to $x_{i}$, obtained by projecting the assignment to the variables of $C_{i}$, returned by the second prover, onto $x_{i}$. (We assume that the answers sent by the second prover always satisfy the clauses appearing in its query). The following theorem is obtained by combining the PCP theorem with the parallel repetition theorem [Raz 1998].

Theorem 7.2 (Arora and Safra [1998], Arora et AL. [1998], and Raz [1998]). There exists a constant $\gamma>0$ such that

-If $\varphi$ is a Yes-Instance, then there is a strategy of the provers for which the acceptance probability is 1.

-If $\varphi$ is a No-Instance, then for any strategy of the provers, the acceptance probability is at most $2^{-\gamma \ell}$.

We denote the set of all random strings of the verifier by $R,|R|=(5 n)^{\ell}$, and the sets of all the possible queries to the first and the second prover by $Q_{1}$ and $Q_{2}$ respectively, $\left|Q_{1}\right|=n^{\ell},\left|Q_{2}\right|=(5 n / 3)^{\ell}$. For each query $q \in Q$, let $A(q)$ be the collection of all possible answers to $q$ (if $q$ is a query of the second prover, then $A(q)$ only contains answers that satisfy all clauses of the query). Let $A=2^{\ell}, A^{\prime}=7^{\ell}$. Then for each $q \in Q_{1},|A(q)|=A$, and for each $q^{\prime} \in Q_{2},\left|A\left(q^{\prime}\right)\right|=A^{\prime}$. Given a random string $r \in R$, let $q_{1}(r), q_{2}(r)$ be the queries sent to the first and the second prover, respectively, when the verifier chooses $r$. Let $N(r)$ denote the set of random strings $r^{\prime} \neq r$, with which $r$ shares a query. In other words, $N(r)=\left\{r^{\prime} \in R \mid r \neq r^{\prime} ; q_{1}(r)=q_{1}\left(r^{\prime}\right)\right.$ or $\left.q_{2}(r)=q_{2}\left(r^{\prime}\right)\right\}$. Note that for each $r \in R,|N(r)|=5^{\ell}+3^{\ell}-1=2^{O(\ell)}$. We denote this number by $N$.

Construction. We now turn to describe our reduction. For each query $q \in Q_{1}$ of the first prover, for each answer $a \in A(q)$, we have an edge $e(q, a)$, whose endpoints are denoted by $v(q, a), u(q, a)$, and whose cost is $(5 / 3)^{\ell}$. We will think of $v(q, a)$ as the first endpoint of $e(q, a)$ and of $u(q, a)$ as its second endpoint, even though the graph is undirected. Similarly, for each query $q \in Q_{2}$ of the second prover, for each answer $a \in A(q)$, there is an edge $e(q, a)=(v(q, a), u(q, a))$, of cost 1 . As before, $v(q, a)$ is called the first endpoint and $u(q, a)$ the second endpoint of $e(q, a)$. Let $E_{0}$ be the set of all resulting edges. For each $q \in Q$, let $V(q)=\{v(q, a), u(q, a) \mid a \in A(q)\}$.

For each random string $r \in R$ of the verifier, we introduce a source-sink pair $s(r), t(r)$, and two collections of edges $E_{1}(r), E_{2}(r)$, whose costs are $\infty$. We also introduce a new set $V(r)$ of vertices, which includes $s(r)$ and $t(r)$. Let $E_{1}=\bigcup_{r \in R} E_{1}(r)$ and $E_{2}=\bigcup_{r \in R} E_{2}(r)$, and let $V^{\prime}=\left(\bigcup_{r \in R} V(r)\right) \cup\left(\cup_{q \in Q_{1} \cup Q_{2}} V(q)\right)$. The final graph consists of the set $V^{\prime}$ of vertices and the set $E_{0} \cup E_{1} \cup E_{2}$ of edges. 

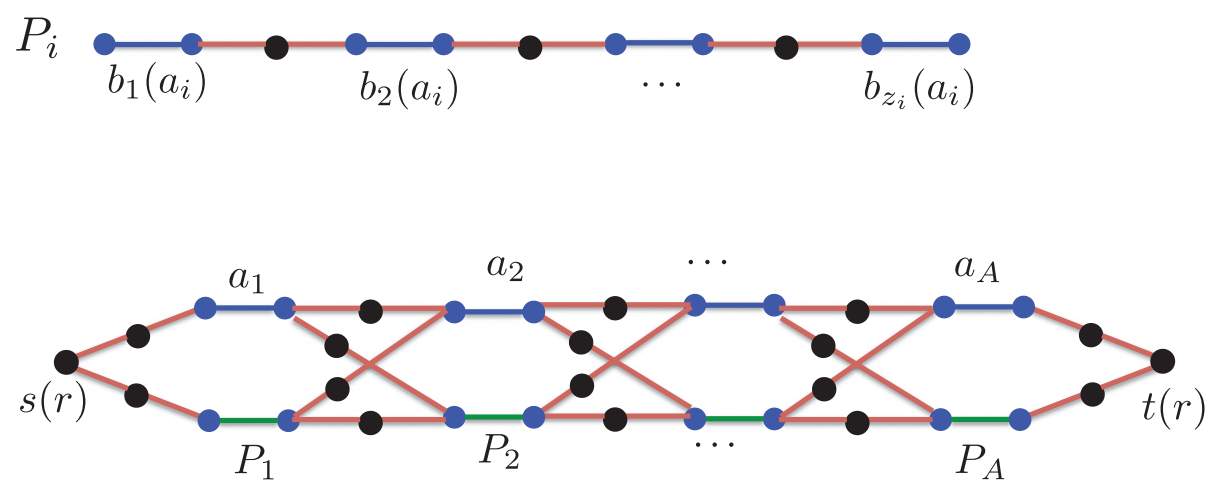

Fig. 7. Graph $G(r)$. Red edges belong to $E_{1}(r)$ and have cost $\infty$. Black vertices belong to $V(r)$.

We now fix some random string $r \in R$ and define the set $E_{1}(r)$ of edges and the set $V(r)$ of vertices. Initially, $V(r)$ only contains the two vertices $s(r)$ and $t(r)$. We start by defining an auxiliary set $E_{1}^{\prime}(r)$ of edges. The final set $E_{1}(r)$ of edges is obtained by subdividing each edge $e \in E_{1}^{\prime}(r)$ with a new vertex $v_{e}$, which is then added to $V(r)$.

We now proceed to define the set $E_{1}^{\prime}(r)$ of edges. Let $q=q_{1}(r), q^{\prime}=q_{2}(r)$. Let $\left(a_{1}, a_{2}, \ldots, a_{A}\right)$ be any ordering of the set $A(q)$ of answers to $q_{1}$. For each $1 \leq i \leq A$, let $b_{1}\left(a_{i}\right), b_{2}\left(a_{i}\right), \ldots, b_{z_{i}}\left(a_{i}\right)$ be the set of all answers to $q^{\prime}$ that are consistent with the answer $a_{i}$ to $q$. We start by connecting the edges corresponding to $b_{1}\left(a_{i}\right), b_{2}\left(a_{i}\right), \ldots, b_{z_{i}}\left(a_{i}\right)$ into a single path $P_{i}$ as follows: for $1 \leq j<z_{i}$, we connect the second endpoint of the edge $e\left(q^{\prime}, b_{j}\left(a_{i}\right)\right)$ to the first endpoint of edge $e\left(q^{\prime}, b_{j+1}\left(a_{i}\right)\right)$. We will refer to $v\left(q^{\prime}, b_{1}\left(a_{i}\right)\right)$ as the first vertex on path $P_{i}$ and to $u\left(q^{\prime}, b_{z_{i}}\left(a_{i}\right)\right)$ as the last vertex. Next, we connect the source $s(r)$ to the first vertex of $e\left(q, a_{1}\right)$ and the first vertex of $P_{1}$. We also connect the second vertex of $e\left(q, a_{A}\right)$ and the last vertex of $P_{A}$ to the sink $t(r)$. Finally, for all $1 \leq i<A$, we connect the last vertex of $P_{i}$ to the first vertices of $e\left(q, a_{i+1}\right)$ and $P_{i+1}$, and the second vertex of $e\left(q, a_{i}\right)$ to the first vertices of $e\left(q, a_{i+1}\right)$ and $P_{i+1}$. This finishes the definition of the set $E_{1}^{\prime}(r)$ of edges.

Our final step is to subdivide each edge $e \in E_{1}^{\prime}(r)$ with a new vertex $v_{e}$. We let $E_{1}(r)$ denote the set of all edges obtained by subdividing the edges of $E_{1}(r)$, and we let $V(r)$ contain $s(r), t(r)$, and all vertices $v_{e}$, where $e \in E_{1}^{\prime}(r)$.

Let $G(r)$ be the graph whose vertex set is $V(q) \cup V\left(q^{\prime}\right) \cup V(r)$, and the edge set consists of $E_{1}(r)$ and the edges of $E_{0}$ representing the answers to $q$ and $q^{\prime}$-that is: $\{e(q, a) \mid a \in A(q)\} \cup\left\{e\left(q^{\prime}, a^{\prime}\right) \mid a^{\prime} \in A\left(q^{\prime}\right)\right\}$. Then $G(r)$ is an "almost layered" graph, where for each $1 \leq i \leq A$, layer $i$ consists of the edge $e\left(q_{1}(r), a_{i}\right)$ and of the path $P_{i}$ (Figure 7). Notice that the only way to disconnect $s(r)$ from $t(r)$ in graph $G(r)$, without deleting edges of $E_{1}(r)$ (whose cost is $\infty$ ), is to delete a pair $e(q, a), e\left(q^{\prime}, a^{\prime}\right)$ of edges, where $a$ and $a^{\prime}$ are matching answers to queries $q$ and $q^{\prime}$, respectively. We note that the size of the set $|V(r)|=2^{O(\ell)}$ is the same for all $r \in R$, and we denote it by $Z$.

Finally, we define the sets $E_{2}(r)$ of edges for all $r \in R$. Given a random string $r \in R$, let $U(r)=\bigcup_{r^{\prime} \in N(r)} V\left(r^{\prime}\right)$. Notice that $|U(r)|=N \cdot Z=2^{O(\ell)}$. We connect $s(r)$ to every vertex in $U(r)$, and we connect every vertex in $U(r)$ to $t(r)$. We denote the resulting set of edges by $E_{2}(r)$, and we set the costs of these edges to be $\infty$. Let $E_{2}=\bigcup_{r^{\prime} \in R} E_{2}(r)$.

Notice that for each pair $r, r^{\prime} \in R$ of random strings, where $r \in N\left(r^{\prime}\right), r^{\prime} \in N(r)$ also holds. Therefore, for every vertex $x \in\{s(r), t(r)\}, y \in\left\{s\left(r^{\prime}\right), t\left(r^{\prime}\right)\right\}$, we add two parallel edges $(x, y)$ in $E_{2}$ : one to $E_{2}(r)$, and one to $E_{2}\left(r^{\prime}\right)$. It will be convenient, instead, to keep only one such edge $(x, y)$, which will be seen as belonging to both $E_{2}(r)$ and $E_{2}\left(r^{\prime}\right)$. The 
degree of $s(r)$ is then exactly $|U(r)|+2$. Two of the edges incident on $s(r)$ belong to $E_{1}(r)$, and all remaining edges belong to $E_{2}$, and connect $s(r)$ to the vertices of $U(r)$. Every vertex that serves as a source or a sink in our graph has exactly the same degree, and we denote it by $\Delta$. Finally, we set the parameter $k$ to be $\Delta-1=2^{O(\ell)}$ - the number of edges of $E_{2}$ incident on each vertex that serves as a source or a sink, plus one. Let $G$ denote this final instance of the VC-kRC problem. We now analyze its properties.

Completeness. Assume first that $\varphi$ is a Yes-Instance, and consider the strategy of the provers that makes the verifier accept with probability 1 . For each query $q \in Q$, let $a(q)$ be the answer to query $q$ under this strategy. Let $E^{\prime}=\{e(q, a(q)) \mid q \in Q\}$. Notice that the cost of $E^{\prime}$ is $(5 / 3)^{\ell} \cdot\left|Q_{1}\right|+\left|Q_{2}\right|=2 \cdot(5 n / 3)^{\ell}$. Denote this cost by $C_{Y I}$. We claim that $E^{\prime}$ is a feasible solution to the VC-kRC instance. Assume otherwise. Then for some random string $r \in R$, there are at least $k$ node-disjoint paths connecting $s(r)$ to $t(r)$ in $G \backslash E^{\prime}$. Let $P_{1}, \ldots, P_{k}$ denote these paths. Since $|U(r)|=k-1$, at least one of these paths does not contain the vertices of $U(r)$. Assume without loss of generality that this path is $P_{1}$. Let $H$ be the graph obtained from $G \backslash E^{\prime}$ by deleting all vertices in $U(r)$ from it. Then there is a path connecting $s(r)$ to $t(r)$ in $H$ - the path $P_{1}$. We assume without loss of generality that $P_{1}$ is a simple path.

Since $a(q), a\left(q^{\prime}\right)$ are matching answers to queries $q$ and $q^{\prime}$, respectively, source $s(r)$ is completely disconnected from sink $t(r)$ in graph $G(r) \backslash E^{\prime}$. Therefore, path $P_{1}$ must use at least one edge that does not belong to $G(r)$. Let $e$ be the first edge of $P_{1}$ that does not belong to $G(r)$. Assume that $e=(x, y)$, where $x$ appears before $y$ as we traverse $P_{1}$ from $s(r)$ to $t(r)$.

Assume first that $x \in V(r)$. It is impossible that $x=s(r)$, as the only edges that $s(r)$ is incident to in graph $H$ are the edges of $E_{1}(r)$, whereas we assumed that $e \notin E_{1}(r)$. Similarly, $x \neq t(r)$. Therefore, $x \in V(r) \backslash\{s(r), t(r)\}$ must hold. But then the only edges incident on $x$ are the edges of $E_{1}(r)$ (which belong to $G_{1}(r)$ ) and the edges of $E_{2}\left(r^{\prime}\right)$ for random strings $r^{\prime} \in N(r)$. We conclude that $e \in E_{2}\left(r^{\prime}\right)$ must hold, for some $r^{\prime} \in N(r)$, and so $y \in\left\{s\left(r^{\prime}\right), t\left(r^{\prime}\right)\right\}$. However, from the definition of $U(r), s\left(r^{\prime}\right), t\left(r^{\prime}\right) \in U(r)$, while $H$ does not contain any vertices of $U(r)$, a contradiction.

Assume now that $x \notin V(r)$. Then it must be an endpoint of an edge $e(q, a)$ for some $q \in\left\{q_{1}(r), q_{2}(r)\right\}, a \in A(q)$. The only edges incident on $x$ that do not belong to $G(r)$ are edges in sets $E_{1}\left(r^{\prime}\right)$, where $r^{\prime} \in N(r)$. However, for such an edge $e$, its other endpoint, $y$, must belong to $V\left(r^{\prime}\right)$, and hence to $U(r)$. Since $H$ does not contain any vertices of $U(r)$, this leads to a contradiction.

Soundness. Assume now that $\varphi$ is a No-Instance, and let $E^{\prime}$ be any solution to the VCkRC instance $G$. We claim that the cost of $E^{\prime}$ must be at least $C_{Y I} \cdot 2^{\gamma \ell / 2} / 16=(5 n / 3)^{\ell}$. $2^{\gamma \ell / 2} / 8$. Assume otherwise. For each query $q \in Q$, let $A^{*}(q)=\left\{a \in A(q) \mid e(q, a) \in E^{\prime}\right\}$. We say that a query $q \in Q$ is bad if and only if $\left|A^{*}(q)\right| \geq 2^{\gamma \ell / 2} / 2$.

Let $Q_{1}^{\prime}$ be the set of all bad queries in $Q_{1}$ and $Q_{2}^{\prime}$ the set of all bad queries in $Q_{2}$. Notice that $\left|Q_{1}\right|<\left|Q_{1}\right| / 4$ : otherwise, $w\left(E^{\prime}\right) \geq(5 / 3)^{\ell} \cdot 2^{\gamma \ell / 2} \cdot\left|Q_{1}^{\prime}\right| / 2=(5 n / 3)^{\ell} \cdot 2^{\gamma \ell / 2} / 8$, a contradiction. Similarly, $\left|Q_{2}^{\prime}\right|<\left|Q_{2}\right| / 4$. Let $R^{\prime} \subseteq R$ be the subset of random strings $r$ for which $q_{1}(r) \notin Q_{1}^{\prime}$ and $q_{2}(r) \notin Q_{2}^{\prime}$. Then $\left|R^{\prime}\right|>|R| / 2$ must hold, as when we choose a random string $r \in R$ uniformly, the probability that $q_{1}(r) \in Q_{1}^{\prime}$ is less than $1 / 4$ and the probability that $q_{2}(r) \in Q_{2}^{\prime}$ is less than $1 / 4$. We now define a strategy for the provers that forces the verifier to accept with probability greater than $2^{-\gamma \ell}$, leading to a contradiction. Given a query $q \in Q_{1}$, if $q \notin Q_{1}^{\prime}$, the first prover randomly chooses an answer from the set $A^{*}(q)$. If $q \in Q_{1}^{\prime}$, the prover returns an arbitrary answer to $q$. Given a query $q \in Q_{2}$, the strategy of the second prover is defined similarly. We now argue that under this strategy of the two provers, the probability of acceptance is greater than $2^{-\gamma \ell}$. Indeed, a probability that a random string $r \in R^{\prime}$ is chosen is at least $\frac{1}{2}$. 
Observe that for each $r \in R^{\prime}$, there are at most $k-1=|U(r)|$ vertex-disjoint paths connecting $s(r)$ to $t(r)$ in graph $G \backslash E^{\prime}$. However, since the edges of $E_{2}(r)$ have infinite cost, they do not belong to $E^{\prime}$. So the pair $s(r)-t(r)$ must be completely disconnected in graph $G(r)$ : otherwise, if $G(r)$ contains a path $P$ connecting $s(r)$ to $t(r)$, then, together with the edges in $E_{2}(r)$, we will obtain $k$ vertex-disjoint paths connecting $s(r)$ to $t(r)$. Therefore, there must be an answer $a \in A^{*}(q)$ to $q$ and an answer $a^{\prime} \in A^{*}\left(q^{\prime}\right)$ that match. The probability that the first prover selects $a$ and the second prover selects $a^{\prime}$ is at least $4 / 2^{\gamma \ell}$. Therefore, overall, the verifier accepts with probability greater than $2^{-\gamma \ell}$, a contradiction.

To summarize, we obtain a gap of $2^{\gamma \ell / 2} / 16$ between the Yes and the No instances while the parameter $k=2^{O(\ell)}$ and the graph size is $n^{\prime}=n^{O(\ell)}$. Therefore, there is a constant $k_{0}$ such that for any constant $k>k_{0}$, we obtain a $k^{\epsilon}$-hardness of approximation for some specific constant $\epsilon$ if $\mathrm{P} \neq \mathrm{NP}$. In general, by setting $\ell=$ poly $\log n$, we get that for any constant $\eta$, for any $k=O\left(2^{\left(\log n^{\prime}\right)^{1-\eta}}\right), k>k_{0}$, there is no $k^{\epsilon}$-approximation algorithm for VC-kRC unless NP $\subseteq$ DTIME $\left(n^{\text {poly } \log n}\right)$.

\section{SINGLE SOURCE-SINK PAIR}

In this section, we study the single source-sink pair version of EC-kRC and VC-kRC, denoted by (st)-EC-kRC and (st)-VC-kRC, respectively. We start with algorithmic results in Section 8.1 and complement them with inapproximability results in Section 8.2.

\subsection{Algorithms for the Single $(s, t)$-Pair Version}

This section is devoted to proving Theorem 1.6. Since we show in Appendix A that VC-kRC captures EC-kRC as a special case, and this reduction remains valid for the single source-sink pair version, it is enough to prove the theorem for VC-kRC. We start with describing the bi-critera approximation algorithm. We reduce the (st)-VC-kRC problem to the problem of finding a minimum-weight vertex (st)-cut in a new graph $G^{\prime}$. Recall that in this problem, we are given a graph $G^{\prime}$, with nonnegative weights $w(v)$ on vertices $v \in V\left(G^{\prime}\right)$, and two special vertices $s$ and $t$. The goal is to find a minimumweight subset $S \subseteq V\left(G^{\prime}\right) \backslash\{s, t\}$ of vertices, whose removal disconnects $s$ from $t$ in $G^{\prime}$. This problem can be solved efficiently by standard techniques.

Given an instance $G$ of the (st)-VC-kRC problem, let OPT denote the value of the optimal solution (that we guess). We produce an instance $G^{\prime}$ of the minimum-weight vertex (st)-cut problem as follows. Graph $G^{\prime}$ is obtained from graph $G$, after subdividing every edge $e \in E(G)$ by a vertex $v_{e}$. The weight of this new vertex is set to be $w\left(v_{e}\right)=w_{e}$, and for each original vertex $v \in V(G)$, we set its weight to $\frac{c}{k-1} \cdot$ OPT.

Assume that we have guessed the value OPT correctly, and let $E^{\prime}$ be the optimal solution to the (st)-VC-kRC problem on graph $G$, with $w\left(E^{\prime}\right)=$ OPT. Then graph $G \backslash E^{\prime}$ contains a subset $S^{\prime} \subseteq V(G) \backslash\{s, t\}$ of at most $(k-1)$ vertices, whose removal disconnects $s$ from $t$. Let $S^{\prime \prime}=\left\{v_{e} \mid e \in E^{\prime}\right\}$ be the subset of vertices of $G^{\prime}$ corresponding to the edges in $E^{\prime}$. Then $S^{\prime} \cup S^{\prime \prime}$ is a feasible solution to the vertex (st)-cut problem in graph $G^{\prime}$, and its value is $w\left(S^{\prime}\right)+w\left(S^{\prime \prime}\right) \leq(k-1) \cdot \frac{c}{k-1} \cdot$ OPT + OPT $\leq(1+c) \cdot$ OPT. Therefore, the value of the minimum-weight (st)-cut in $G^{\prime}$ is at most $(1+c)$. OPT. On the other hand, let $S$ be the minimum-weight vertex (st)-cut in graph $G^{\prime}$. Partition $S$ into two subsets: $S^{\prime}=S \cap V(G)$ is the subset of vertices in the original graph $G$, and $S^{\prime \prime}=S \backslash S^{\prime}$ is the set of all remaining vertices. Let $E^{\prime}=\left\{e \mid v_{e} \in S^{\prime \prime}\right\}$ be the corresponding subset of edges in graph $G$. Then $\left|S^{\prime}\right| \leq \frac{\mathrm{OPT} \cdot(1+c)}{c \cdot \mathrm{OPT} /(k-1)} \leq(k-1)(1+1 / c)<k(1+1 / c)$. Therefore, $E^{\prime}$ is a $((1+1 / c),(1+c))$-bi-criteria approximate solution.

To obtain a factor $(k+1)$-approximation algorithm, we use the preceding algorithm, setting the parameter $c=k$. Using the preceding analysis, the value of the minimum-weight node (st)-cut in graph $G^{\prime}$ is at most $(k+1) \cdot$ OPT. Moreover, since 
$\left|S^{\prime}\right| \leq(k-1)(1+1 / k)=k-1+\frac{k-1}{k}<k$, set $E^{\prime}$ of edges is indeed a feasible solution for the $\mathrm{V} C-\mathrm{kRC}$ instance $G$.

\subsection{Inapproximability of (st)-VC-kRC}

In this section, we complement our upper bounds from Section 8.1 by inapproximability results and prove Theorems 1.7, 1.8, and 1.9. The starting point for all three reductions is similar. We define the SSVE problem and show an approximation preserving reduction from SSVE to (st)-VC-kRC in Section 8.2.1. We then show inapproximability results for SSVE in subsequent sections, which are used to establish the lower bounds on the approximability of (st)-VC-kRC.

\subsubsection{Small Set Vertex Expansion.}

Definition 8.1 (Small Set Vertex Expansion Problem). Given a bipartite graph $G=(U, V, E)$ and a parameter $0<\alpha \leq 1$, the aim is to find a subset $S \subseteq U$ of vertices, $|S| \geq \alpha|U|$, minimizing the number of its neighbors, $|\Gamma(S)|$.

We present a gap-preserving reduction from SSVE to (st)-VC-kRC, which will allow us to later focus on proving inapproximability of SSVE.

THEOREM 8.2. Let $G=(U, V, E)$ be any bipartite graph with $|U|=m,|V|=n$, and let $N=2 m n+1$. We can efficiently construct an edge-weighted graph $G^{\prime}$ with two special vertices $s, t \in V\left(G^{\prime}\right)$ such that for any $0<\alpha<1$ and any integer $0 \leq C \leq|V|$, the following property holds: there is a subset $S \subseteq U$ in graph $G$ with $|S| \geq \alpha|U|$ and $|\Gamma(S)| \leq C$ if and only if there is a solution of cost at most $C \cdot N$ to the (st)-VC-kRC problem on graph $G^{\prime}$, where the parameter $k$ is set to be $k=|U|(1-\alpha)+1$.

Proof. Given an SSVE instance $G=(U, V, E)$, with $|U|=m,|V|=n$, let $N=$ $2 n m+1$. To construct the graph $G^{\prime}$, we start with the bipartite graph $G=(U, V, E)$ and then replace every vertex $v \in V$ with a clique $K(v)$ on $N$ new vertices. All edges of the clique $K(v)$ have cost $\infty$. Let $V^{\prime}=\bigcup_{v \in V} V(K(v))$ be the set of all vertices in all such cliques. We add an edge of cost $\infty$ between $u \in U$ and every vertex in $K(v)$ if $(u, v) \in E(G)$. We also add two additional vertices $s$ and $t$. For every vertex $u \in U$, add an $\infty$-cost edge $(s, u)$, and for every $v^{\prime} \in V^{\prime}$, add a cost- 1 edge $\left(v^{\prime}, t\right)$ to $G^{\prime}$. This completes the description of graph $G^{\prime}$. Given a parameter $0<\alpha<1$, we set $k=|U|(1-\alpha)+1$.

Completeness. Suppose that we have a subset $S \subseteq U$ with $|S|=\alpha|U|$ and $|\Gamma(S)| \leq C$ in graph $G$. We construct a solution to the (st)-VC-kRC instance $G^{\prime}$ as follows: for each vertex $v \in \Gamma(S)$, we add all edges between the vertices of $K(v)$ and the vertex $t$ to the solution. Let $E^{*}$ denote the resulting set of edges. Then $\left|E^{*}\right| \leq C N$. We now argue that $E^{*}$ is a valid $k$-route (st)-cut. Indeed, consider the graph $G^{\prime} \backslash E^{*}$, and let $S^{\prime}=U \backslash S$. Then $\left|S^{\prime}\right|=k-1$, and once the vertices of $S^{\prime}$ are removed from $G^{\prime}$, no paths connecting $s$ to $t$ remain in the graph.

Soundness. Assume now that we have a solution $E^{*}$ of cost at most $C N$ to the (st) VC-kRC instance $G^{\prime}$. Notice that all edges in $E^{*}$ must be incident on $t$, as all other edges have cost $\infty$.

Our first step is to transform the solution $E^{*}$ so that for each vertex $v \in V$, either all edges connecting the vertices of $K_{v}$ to $t$ belong to the solution or none of them. To perform this transformation, we consider the vertices $v \in V$ one by one. For each such vertex $v$, let $E_{v}$ be the set of all edges connecting the vertices of $K(v)$ to $t$. If $\left|E_{v} \backslash E^{*}\right| \geq k$, then we remove all edges of $E_{v}$ from $E^{*}$. Otherwise, we add all edges of $E_{v} \backslash E^{*}$ to $E^{*}$. We first claim that the resulting set of edges remains a valid solution to the (st)-VC-kRC problem. Indeed, let $E^{*}$ be the subset of edges in the solution before the vertex $v \in V$ is processed, and assume that $E^{*}$ is a valid $k$-route cut. Assume that $\left|E_{v} \backslash E^{*}\right| \geq k$. 
Partition the vertices in $K(v)$ into two subsets: $V_{1}$ is the subset of vertices $v^{\prime}$ whose edge $\left(v^{\prime}, t\right) \in E^{*}$, and $V_{2}$ denotes the set of the remaining vertices. Notice that $\left|V_{2}\right| \geq k$ must hold. We claim that $E^{*} \backslash E_{v}$ remains a valid solution to the $k$-route cut instance. Assume otherwise. Then graph $G^{\prime} \backslash\left(E^{*} \backslash E_{v}\right)$ has $k$ vertex-disjoint paths $P_{1}, \ldots, P_{k}$, connecting $s$ to $t$. We can assume without loss of generality that each such path contains at most one vertex of $K(v)$, as each vertex in $K(v)$ is directly connected to $t$ by an edge in graph $G^{\prime} \backslash\left(E^{*} \backslash E_{v}\right)$. For $1 \leq i \leq k$, let $v_{i} \in K(v)$ be the vertex lying on $P_{i}$ (if it exists). We now construct $k$ vertex-disjoint paths $P_{1}^{\prime}, \ldots, P_{k}^{\prime}$, connecting $s$ to $t$ in graph $G^{\prime} \backslash E^{*}$, reaching a contradiction. Each path $P_{i}^{\prime}$ is constructed from path $P_{i}$, by replacing the vertex $v_{i}$ with some vertex of $V_{2}$ so that each vertex of $V_{2}$ appears on at most one such path. This can be done since $\left|V_{2}\right| \geq k$.

Let $E^{* *}$ be the subset of edges obtained from $E^{*}$ after we process all vertices $v \in V$. From the preceding discussion, $E^{* *}$ is a feasible solution to the (st)-VC-kRC instance $G^{\prime}$. Moreover, $\left|E^{* *} \backslash E^{*}\right| \leq n k \leq 2 m n<N$, and $\left|E^{* *}\right|$ is an integral multiple of $N$. Therefore, if $\left|E^{*}\right| \leq C N$, where $\bar{C}$ is an integer, $\left|E^{* *}\right| \leq C N$ as well.

Since $E^{* *}$ is a valid $k$-route cut in $G^{\prime}$, there is a set $S^{\prime}$ of $(k-1)$ vertices, whose removal from $G^{\prime} \backslash E^{* *}$ separates $s$ from $t$. Since for each $v \in V$, either $E_{v} \subseteq E^{* *}$, or $E_{v} \cap E^{* *}=\emptyset$, while $|K(v)|>2 k$, we can assume without loss of generality that $\bar{S}^{\prime} \subseteq U$. Let $S=U \backslash S^{\prime}$. Then $|S| \geq \alpha m$, and the set $\Gamma(S)$ is contained in the set $\left\{v \in V \mid \bar{E}_{v} \subseteq E^{* *}\right\}$, implying that $|\Gamma(S)| \leq C$.

8.2.2. Inapproximability from the Random $\kappa$-AND Assumption. This section is devoted to proving Theorem 1.7. We prove the following inapproximability result for SSVE.

THEOREM 8.3. For every large enough constant $\kappa$, there are parameters $\alpha, \beta$ that depend on $\kappa$ only such that assuming Hypothesis 1.1, no polynomial-time algorithm, given a bipartite graph $G=(U, V, E)$, can distinguish between the following two cases:

-Completeness: There is a subset $S \subseteq U$, with $|S|=\alpha|U|$ and $|\Gamma(S)| \leqq \beta|V|$, and -Soundness: For any subset $S \subseteq U$ with $|S| \geq \frac{\alpha}{2^{\kappa / 2}}|U|,|\Gamma(S)|>\beta \cdot 2^{\sqrt{\kappa} / c}|V|$,

where $c$ is a constant independent of $\kappa$.

Combining Theorem 8.3 with Theorem 8.2, we get that there is no polynomial-time algorithm for (st)-VC-kRC that distinguishes between the cases where there is a solution of $\operatorname{cost} \beta|V| \cdot N$ for parameter $k=|U|(1-\alpha)+1$ and the cases where there is no solution of $\operatorname{cost} \beta / 2^{\sqrt{\kappa} / c}$ and parameter $k=|U|\left(1-\frac{\alpha}{2^{\kappa / 2}}\right)+1$. Since $\alpha$ and $\kappa$ are constants, this will complete the proof of Theorem 1.7 .

Proof. The proof proceeds in two steps. In the first step, we show a simple reduction that gives a weak inapproximability result for the SSVE problem. Next, we amplify the inapproximability factor by using graph products. The first step is summarized in the next lemma.

LEMMA 8.4. Let $0<\delta<\frac{1}{2}$ be any parameter, and $\kappa>\kappa_{0}$ any large enough constant, where $\kappa_{0}$ depends on $\delta$. Assuming Hypothesis 1.1, there is no polynomial time algorithm that, given a bipartite graph $G=(U, V, E)$, can distinguish between the following two cases:

-Completeness: There is a subset $S \subseteq U$, with $|S|=|U| / 2^{c_{0} \sqrt{\kappa}}$ and $|\Gamma(S)| \leq|V| / 2$, and -Soundness: For any subset $S \subseteq U$, with $|S| \geq|U| / 2^{\kappa(1-2 \delta)},|\Gamma(S)|>(1+\delta) \cdot|V| / 2$,

(here $c_{0}$ is the constant from Hypothesis 1.1).

Proof. We start with the following simple claim about random instances of the $\kappa$-AND problem. 
Claim 8.1. Let $0<\delta<\frac{1}{2}$ be any parameter, $\kappa>\kappa_{0}$ a large enough constant, where $\kappa_{0}$ depends on $\delta$, and $\Delta \geq \Delta_{0}$ a large enough constant, where $\Delta_{0}$ depends on $\kappa$ and $\delta$. Then for any random $\kappa-A N D$ formula $\Phi$ on $n$ variables and $m=\Delta n$ clauses, every subset of $m \cdot 2^{2 \delta \kappa} / 2^{\kappa}$ clauses in $\Phi$ contains at least $(1+\delta) n$ different literals, with high probability.

Proof. Fix any set $S$ of $(1+\delta) n$ different literals. We say that a clause $C$ of $\Phi$ is bad for $S$ if and only if all literals of $C$ belong to $S$. Let $\mathcal{E}(C, S)$ denote the event that $C$ is bad for $S$. Then

$$
\operatorname{Pr}[\mathcal{E}(C, S)]=\left(\frac{1+\delta}{2}\right)^{\kappa}<2^{1.5 \delta \kappa} / 2^{\kappa} .
$$

Therefore, the expected number of clauses $C \in \Phi$ that are bad for $S$ is at most $\mu=m \cdot 2^{1.5 \delta \kappa} / 2^{\kappa}$. We say that a bad event $\mathcal{E}(S)$ happens if at least $m \cdot 2^{2 \delta \kappa} / 2^{\kappa}$ clauses of $\Phi$ have their literals contained in $S$. Notice that $m \cdot 2^{2 \delta \kappa} / 2^{\kappa}=\mu \cdot 2^{0.5 \delta \kappa}=\mu\left(1+\delta^{\prime}\right)$ for some constant $\delta^{\prime}$ that depends on $\delta$ and $\kappa$. Therefore, by Chernoff bounds,

$$
\operatorname{Pr}[\mathcal{E}(S)] \leq e^{-\mu \delta^{\prime 2} / 2} .
$$

Let $\mathcal{E}$ be the event that for any subset $S$ of $(1+\delta) n$ literals, the bad event $\mathcal{E}(S)$ happens. Since the total number of subsets $S$ of literals is at most $2^{2 n}$, using the union bound,

$$
\operatorname{Pr}[\mathcal{E}] \leq 2^{2 n} \cdot e^{-\mu \delta^{\prime 2} / 2}=2^{2 n} \cdot 2^{-c^{\prime} \cdot m}
$$

where $c^{\prime}$ is some constant that depends on $\delta$ and $\kappa$. Clearly, setting $\Delta=4 / c^{\prime}$ ensures that $\operatorname{Pr}[\mathcal{E}] \leq 2^{-2 n}$.

Given a Random $\kappa$-AND instance $\Phi$ with $n$ variables and $m=\Delta n$ variables (where $\Delta$ is chosen as in Claim 8.1), we define the corresponding bipartite graph $G=(U, V, E)$ as follows. Let $U$ be the set of clauses, and let $V$ be the set of $2 n$ literals. Connect each clause $C$ and literal $\ell$ with an edge if and only if $\ell$ belongs to $C$.

For the completeness case, if $\Phi$ is $\left(2^{-c_{0} \sqrt{\kappa}}\right)$-satisfiable, let $S$ be the set of $m / 2^{c_{0} \sqrt{\kappa}}$ clauses that can be satisfied by some assignment. It is easy to see that $\Gamma(S)$ never contains both a literal and its negation. Therefore, $|\Gamma(S)| \leq n=|V| / 2$.

For the soundness case, by Claim 8.1, with high probability over the choice of the $\kappa$-AND formula $\Phi$, for every set $S \subseteq V$ of size $|S|=m / 2^{\kappa(1-2 \delta)}$, we have $|\Gamma(S)|>$ $(1+\delta) \cdot n=(1+\delta)|V| / 2$. Therefore, an efficient algorithm that distinguishes between the completeness and the soundness settings can be used to refute random $\kappa$-AND formulas.

We say that a bipartite graph $G=(U, V, E)$ is $(\alpha, \beta)$-expanding if and only if for every $S \subseteq U$, with $|S| \geq \alpha|U|$, we have $|\Gamma(S)|>\beta|V|$. From Lemma 8.4, assuming Hypothesis 1.1, no polynomial-time algorithm can distinguish between bipartite graphs that are $\left(2^{-\kappa(1-2 \delta)}, \frac{1+\delta}{2}\right)$-expanding, and graphs that are not $\left(2^{-c_{0} \sqrt{\kappa}}, \frac{1}{2}\right)$-expanding, for any constant $0<\delta<\frac{1}{2}$ and any large enough constant $\kappa>\kappa_{0}$, where $\kappa_{0}$ depends on $\delta$. Next, we will use tensor product of bipartite graphs to amplify this gap.

Definition 8.5 (Tensor Product of Bipartite Graphs). For two graphs $G_{1}=\left(U_{1}, V_{1}, E_{1}\right)$, $G_{2}=\left(U_{2}, V_{2}, E_{2}\right)$, let $G=G_{1} \otimes G_{2}$ be the bipartite graph $G=(U, V, E)$, where $U=$ $U_{1} \times U_{2}, V=V_{1} \times V_{2}$, and

$E=\left\{\left(\left(u_{1}, u_{2}\right),\left(v_{1}, v_{2}\right)\right) \mid\left(u_{1}, u_{2}\right) \in U_{1} \times U_{2},\left(v_{1}, v_{2}\right) \in V_{1} \times V_{2},\left(u_{1}, v_{1}\right) \in E_{1},\left(u_{2}, v_{2}\right) \in E_{2}\right\}$.

For $k \in \mathbb{Z}^{+}$, define $G^{\otimes k}$ inductively as follows: $G^{\otimes 2}=G \otimes G$, and for $k>2, G^{\otimes k}=$ $G^{\otimes(k-1)} \otimes G$. 
The following lemma shows that the tensor product can be used to amplify the expansion gap, with a small loss in the threshold parameter $\alpha$.

Lemma 8.6 [Tensor Product Amplification]. Let $G=(U, V, E)$ be any bipartite graph, and $0<\alpha, \beta<1$ parameters:

-If $G$ is not $(\alpha, \beta)$-expanding, then $G^{\otimes 2}$ is not $\left(\alpha^{2}, \beta^{2}\right)$-expanding.

-If $G$ is $(\alpha, \beta)$-expanding, then $G^{\otimes 2}$ is $\left(2 \alpha-\alpha^{2}, \beta^{2}\right)$-expanding and therefore is $\left(2 \alpha, \beta^{2}\right)$ expanding.

Proof. Let $G^{\otimes 2}=\left(U^{\prime}, V^{\prime}, E^{\prime}\right)$, and let $m=|U|, n=|V|$. Then $\left|U^{\prime}\right|=m^{2},\left|V^{\prime}\right|=n^{2}$. Assume first that $G$ is not ( $\alpha, \beta)$-expanding. Then there is a subset $S \subseteq U$ of vertices, $|S|=\alpha m$, such that $\left|\Gamma_{G}(S)\right| \leq \beta n$. Consider the subset $S \times S$ of vertices in graph $G^{\oplus 2}$. Then $|S \times S|=\alpha^{2} m^{2}$, and $\left|\Gamma_{G^{\otimes 2}}(S \times S)\right|=|\Gamma(S) \times \Gamma(S)| \leq \beta^{2} n^{2}$. Therefore, graph $G^{\otimes 2}$ is not $\left(\alpha^{2}, \beta^{2}\right)$-expanding.

Assume now that $G$ is $(\alpha, \beta)$-expanding, and assume for contradiction that $G^{\otimes 2}$ is not $\left(2 \alpha-\alpha^{2}, \beta^{2}\right)$-expanding. Let $S^{\prime} \subseteq U \times U$ be a subset of $V^{\prime}$ with $\left|S^{\prime}\right|=\left(2 \alpha-\alpha^{2}\right) m^{2}$, and $\left|\Gamma_{G^{\otimes 2}}\left(S^{\prime}\right)\right| \leq \beta^{2} n^{2}$. For each $i \in \bar{U}$, let $S_{i}^{\prime}=\left\{j \mid(i, j) \in S^{\prime}\right\}$. Call $i \in U$ good if and only if $\left|S_{i}^{\prime}\right| \geq \alpha|U|$. Let $T \subseteq U$ be the set of all good vertices. By an averaging argument, we get that

$$
\left|S^{\prime}\right| \leq|T| \cdot m+(m-|T|) \cdot \alpha m .
$$

Therefore, $|T| \geq \frac{\left|S^{\prime}\right|-\alpha m^{2}}{(1-\alpha) m} \geq \frac{\left(2 \alpha-\alpha^{2}\right) m^{2}-\alpha m^{2}}{(1-\alpha) m}=\alpha m$.

On the other hand, for each $i \in T$, the number of neighbors of the vertices in $\{i\} \oplus S_{i}^{\prime}$ in graph $G^{\oplus 2}$ is at least $\left|\Gamma_{G}(i)\right| \cdot\left|\Gamma_{G}\left(S_{i}\right)\right| \geq\left|\Gamma_{G}(i)\right| \cdot \beta n$ (since $\left|S_{i}\right| \geq \alpha m$, and graph $G$ is $(\alpha, \beta)$-expanding). Therefore, $\left|\Gamma_{G^{\otimes 2}}\left(S^{\prime}\right)\right| \geq\left|\Gamma_{G}(T)\right| \cdot \beta n>\beta^{2} n^{2}$, a contradiction to $S^{\prime}$ being a violating set.

We are now ready to complete the proof of Theorem 8.3. We start with the instances given by Lemma 8.4, and repeatedly apply Lemma 8.6 to them. Specifically, let $G_{0}$ be the graph obtained from Lemma 8.4, and for $i>0$, let $G_{i}=G_{i-1}^{\otimes 2}$. Our final graph is $G_{\ell}$, where $\ell=\log \left(\sqrt{\kappa} / 6 c_{0}\right)$.

Assume first that the initial graph $G_{0}$ is a YES-instance-that is, $G_{0}$ is not $\left(\alpha_{0}, \beta_{0}\right)$ expanding, for $\alpha_{0}=2^{-c_{0} \sqrt{\kappa}}, \beta_{0}=\frac{1}{2}$. Then by Lemma 8.6, graph $G_{\ell}$ is not $\left(\alpha_{\ell}, \beta_{\ell}\right)$ expanding, where $\alpha_{\ell}=\alpha_{0}^{2^{\ell}}=2^{-c_{0} \sqrt{\kappa} \cdot \sqrt{\kappa} / 6 c_{0}}=2^{-\kappa / 6}$, and $\beta_{\ell}=\beta_{0}^{2^{\ell}}=2^{-\sqrt{\kappa} / 6 c_{0}}$.

Assume now that the initial graph $G_{0}$ is a NO-instance-that is, $G_{0}$ is $\left(\alpha_{0}, \beta_{0}\right)$ expanding, for $\alpha_{0}=2^{-k(1-2 \delta)}, \beta_{0}=(1+\delta) / 2$. Then by Lemma 8.6, graph $G_{\ell}$ is $\left(\alpha_{\ell}, \beta_{\ell}\right)$ expanding, for $\alpha_{\ell}=2^{\ell} \alpha_{0}=\frac{\sqrt{\kappa}}{6 c_{0} \cdot 2^{k(1-2 \delta)}}$, and $\beta_{\ell}=\beta_{0}^{2^{\ell}}=\left(\frac{1+\delta}{2}\right)^{\sqrt{\kappa} / 6 c_{0}}$.

Let us now set $\delta=1 / 18$, and denote $\alpha=2^{-\kappa / 6}$ and $\beta=2^{-\sqrt{\kappa} / 6 c_{0}}$. Denote $G_{\ell}=$ $(U, V, E)$, with $|U|=m,|V|=n$. We then get that in the YES-instance, there is a subset $S \subseteq U$ of at least $\alpha|U|$ vertices such that $|\Gamma(S)| \leq \beta|V|$. For the NO-instance, for any subset $S \subseteq U$ with $|S| \geq \alpha|U| / g_{1}$, we have that $|\Gamma(S)|>\beta|V| \cdot g_{2}$. It now remains to bound $g_{1}$ and $g_{2}$. First,

$$
g_{1}=\frac{1}{2^{\kappa / 6}} / \frac{\sqrt{\kappa}}{6 c_{0} \cdot 2^{\kappa(1-2 \delta)}} \geq \frac{2^{\kappa(1-3 \delta)}}{2^{\kappa / 6}} \geq 2^{\kappa / 2}
$$

if $\kappa$ is large enough and $\delta=1 / 18$.

Finally,

$$
g_{2}=\left(\frac{1+\delta}{2}\right)^{\sqrt{\kappa} / 6 c_{0}} / 2^{-\sqrt{\kappa} / 6 c_{0}}=(1+\delta)^{\sqrt{\kappa} / 6 c_{0}} .
$$

This completes the proof of the bi-criteria hardness in Theorem 8.3. 
8.2.3. Inapproximability from the Random 3SAT Assumption. In this section, we focus on proving Theorem 1.8. We use the following restatement of Theorem 2 of Feige [2002].

Theorem 8.7. For every fixed $\epsilon>0$, for a $\Delta$ sufficiently large constant independent of $n$, assuming Hypothesis 1.2, there is no polynomial-time algorithm that, given a random $3 A N D$ formula on $n$ variables and $m=\Delta$ n clauses, returns "typical" with probability $\frac{1}{2}$, but never returns "typical" if the formula is $\left(\frac{1}{4}-\epsilon\right)$-satisfiable.

As before, we start by proving a bi-criteria hardness result for the SSVE problem.

Theorem 8.8. Given a bipartite graph $G=(U, V, E)$, assuming Hypothesis 1.2, no polynomial time algorithm can distinguish between the following two cases:

-Completeness: There is a subset $S \subseteq U$ with $|S| \geq|U| / 5$ and $|\Gamma(S)| \leq|V| / 2$, and -Soundness: For every subset $S \subseteq U$, if $|S| \geq|U| / 6$, then $|\Gamma(S)| \geq 11|V| / 20$.

Theorem 1.8 then follows immediately by combining Theorem 8.2 with Theorem 8.8 . We now focus on proving Theorem 8.8. As before, we first prove a simple fact about random 3AND formulas that will lead to the proof of the theorem.

Claim 8.2. For sufficiently large $\Delta$, with high probability, every set of $m / 6$ clauses in an R3AND instance with $m=\Delta$ clauses contains at least 1.1 different literals.

Proof. Fix a set $S$ of $1.1 n$ different literals. For a 3 AND clause $C$, let $\mathcal{E}(S, C)$ be the event that all three literals of $C$ are contained in $S$. Clearly, $\operatorname{Pr}[\mathcal{E}(S, C)]=\left(\frac{1.1}{2}\right)^{3} \leq$ $1 / 6-c$ for some constant $c>0$. Therefore, the expected number of clauses contained in $S$ is at most $(1 / 6-c) m$. Let $\mathcal{E}(S)$ be the bad event that at least $m / 6$ clauses are contained in $S$.

By Chernoff bound,

$$
\operatorname{Pr}[\mathcal{E}(S)]<2^{-c^{\prime} m}
$$

for some small constant $c^{\prime}>0$.

Let $\mathcal{E}$ be the event that $\mathcal{E}(S)$ happens for at least one subset $S$ of $1.1 n$ literals. Since the number of such possible subsets $S$ is bounded by $2^{2 n}$, when $\Delta>3 / c^{\prime}$, by a union bound, we have that

$$
\operatorname{Pr}[\mathcal{E}]<2^{-c^{\prime} m} \cdot 2^{2 n}<2^{-n}
$$

Given a random 3 AND formula $\Phi$, we construct a graph $G=(U, V, E)$, where $U$ contains a vertex $u_{C}$ for each clause $C \in \Phi$ and $V$ contains a vertex $v_{\ell}$ for each literal $\ell$. We add an edge $\left(u_{C}, v_{\ell}\right)$ if and only if literal $\ell$ belongs to clause $C$. Assume for contradiction that there is a polynomial-time algorithm $\mathcal{A}$, distinguishing between instances where there is a subset $S \subseteq U$ with $|S| \geq|U| / 5$ and $|\Gamma(S)| \leq|V| / 2$, and instances where for every subset $S \subseteq \bar{U}$, if $|S| \geq|U| / 6$, then $|\Gamma(S)| \geq 11|\bar{V}| / 20$. Given a random 3AND formula $\Phi$, we apply algorithm $\mathcal{A}$ to the resulting graph $G$. If the algorithm establishes that we are in the second scenario (i.e., for each subset $S$ with $|S| \geq|U| / 6, \Gamma(S) \geq 11|V| / 20)$, then we output "typical." From Claim 8.2, this will happen most of the time. However, if the formula $\Phi$ is $\left(\frac{1}{4}-\epsilon\right)$-satisfiable, then we can let $S$ be the set of satisfied clauses, and since $\Gamma(S)$ cannot contain a literal and its negation, we will get that $|\Gamma(S)| \leq|V| / 2$. Therefore, using Theorem 8.7, algorithm $\mathcal{A}$ can be used to refute Hypothesis 1.2 .

8.2.4. Reduction from the Densest $\kappa$-Subgraph Problem. In this section, we prove Theorem 1.9. As before, we do so by proving a similar result for the SSVE problem. 
THEOREM 8.9. For any constant $\lambda \geq 2$, and for any approximation factor $\rho$ (that may depend on $n$ ), if there is an efficient factor $\rho$ approximation algorithm for the SSVE problem, then there is an efficient factor $\left(2 \rho^{\lambda}\right)$-approximation algorithm for the $\lambda$-uniform hypergraph densest $\kappa$-subgraph problem.

Observe that combining Theorem 8.9 with Theorem 8.2 immediately implies Theorem 1.9. We now focus on proving Theorem 8.9.

Proof. Given a $\lambda$-uniform hypergraph densest $\kappa$-subgraph instance $G=(V, E)$, we construct an instance $G^{\prime}=\left(U^{\prime}, V^{\prime}, E^{\prime}\right)$ of SSVE as follows. For each hyperedge $e \in E$, we add a vertex $u_{e}$ to $U^{\prime}$. The set $V^{\prime}$ of vertices is $V^{\prime}=V$. We add an edge between $u_{e} \in U^{\prime}$ and $v \in V^{\prime}$ if and only if vertex $v$ belongs to the hyperedge $e$. Since $\lambda$ is a constant, we can assume that $\kappa \gg \lambda^{2}$ (otherwise, the optimal solution to the densest $\kappa$-subgraph instance can be found efficiently by exhaustive search).

Let $\mathcal{A}$ be a factor $\rho$ approximation algorithm for the SSVE problem. We now show a factor $2 \rho^{\lambda}$-approximation algorithm for the densest $\kappa$-subgraph problem. The algorithm will guess the value $m^{\prime}$ of the optimal solution to the densest $k$-subgraph instance $G$. It will then apply algorithm $\mathcal{A}$ to instance $G^{\prime}$, with value $\alpha=m^{\prime} /\left|U^{\prime}\right|$. If value $m^{\prime}$ was guessed correctly, then there is a subset $S \subseteq U^{\prime}$ of vertices, with $|S|=m^{\prime}$, and $|\Gamma(S)|=\kappa$. Therefore, algorithm $\mathcal{A}$ must return a subset $S^{\prime} \subseteq U^{\prime}$ of vertices, with $\left|S^{\prime}\right|=m^{\prime}$, and $\left|\Gamma\left(S^{\prime}\right)\right| \leq \kappa \cdot \rho$. Let $V^{\prime}=\Gamma\left(S^{\prime}\right)$. Set $V^{\prime}$ is also a subset of vertices in the initial instance $G$, and we are now guaranteed that $\left|V^{\prime}\right| \leq \kappa \cdot \rho$ while the number of edges contained in $V^{\prime}$ is at least $m^{\prime}$. Let $V^{\prime \prime}$ be a random subset of $\kappa$ vertices from $V^{\prime}$. Observe that for a hyperedge $e \subseteq V^{\prime}$, the probability that $e$ is contained in $V^{\prime \prime}$ is at least $\left(\frac{\kappa-\lambda}{\rho \kappa}\right)^{\lambda} \geq \frac{2}{3} \cdot\left(\frac{1}{\rho}\right)^{\lambda}\left(\right.$ since $\left.\kappa \gg \lambda^{2}\right)$, and so the expected number of hyperedges contained in $V^{\prime \prime}$ is at least $2 m^{\prime} / 3 \rho^{\lambda}$.

\section{APPENDIX}

\section{A CONNECTION BETWEEN VC-kRC AND EC-kRC}

In this section, we show that EC-kRC can be cast as a special case of VC-kRC. Indeed, assume that we are given an instance $G=(V, E)$ of EC-kRC with costs $w_{e}$ on edges $e \in E$, a set $\left\{\left(s_{i}, t_{i}\right)\right\}_{i=1}^{r}$ of source-sink pairs, and an integer $k$. We construct an instance $G^{\prime}=\left(V^{\prime}, E^{\prime}\right)$ of the VC-kRC problem as follows. For each vertex $u \in V$, for each edge $e$ incident on $u$ in $G$, we create a new vertex $v(u, e)$ in graph $G^{\prime}$. Each pair $v(u, e), v\left(u, e^{\prime}\right)$ of such vertices is connected with an edge of cost $\infty$. In other words, we replace the vertex $u$ with a clique of $d(u)$ vertices, where $d(u)$ is the degree of $u$ in $G$, and set the weights of the edges in the clique to be $\infty$. Let $K(u)$ denote this clique, and let $V(u)$ and $E(u)$ denote the sets of its vertices and edges, respectively. Let $E_{1}=\bigcup_{u \in V} E(u)$. We now define another set $E_{2}$ of edges, corresponding to the original edges in graph $G$. For each edge $e=\left(u, u^{\prime}\right) \in E$, we add an edge $\left(v(u, e), v\left(u^{\prime}, e\right)\right)$ of weight $w_{e}$ to $E_{2}$. Graph $G^{\prime}=\left(V^{\prime}, E^{\prime}\right)$ is then defined as $V^{\prime}=\bigcup_{u \in V} V(u)$, and $E^{\prime}=E_{1} \cup E_{2}$. To define the source-sink pairs, we select, for each $1 \leq i \leq r$, an arbitrary vertex $s_{i}^{\prime} \in V\left(s_{i}\right)$, and an arbitrary vertex $t_{i}^{\prime} \in V\left(t_{i}\right)$, and we let $\left(s_{i}^{\prime}, t_{i}^{\prime}\right)$ be a source-sink pair in the new instance. Therefore, the set of the source-sink pairs becomes $\left\{\left(s_{i}^{\prime}, t_{i}^{\prime}\right)\right\}_{i=1}^{r}$. The parameter $k$ remains unchanged. We now show that the two instances are equivalent in the sense that any feasible solution $E^{*}$ to the EC-kRC instance $G$ implies a feasible solution $E^{* *}$ to the VC-kRC instance $G^{\prime}$ and vice versa.

Let $E^{*}$ be any feasible solution to the EC-kRC instance $G$. We claim that there is a solution $E^{* *}$ to the VC-kRC instance $G^{\prime}$, of the same weight. The solution $E^{* *}$ contains, for each edge $e=\left(u, u^{\prime}\right) \in E^{*}$, the corresponding edge $\left(v(u, e), v\left(u^{\prime}, e\right)\right)$ of $E^{\prime}$. Clearly, 
the weight of $E^{* *}$ is the same as the weight of $E^{*}$. We now claim that $E^{* *}$ is a feasible solution to the VC-kRC instance $G^{\prime}$. Assume otherwise, and let $\left(s_{i}^{\prime}, t_{i}^{\prime}\right)$ be any source-sink pair such that $G^{\prime} \backslash E^{* *}$ contains at least $k$ node-disjoint paths $P_{1}^{\prime}, \ldots, P_{k}^{\prime}$ connecting $s_{i}^{\prime}$ to $t_{i}^{\prime}$. We show that graph $G \backslash E^{*}$ must then contain at least $k$ edge-disjoint paths $P_{1}, \ldots, P_{k}$, connecting $s_{i}$ to $t_{i}$, leading to a contradiction. For $1 \leq j \leq k$, path $P_{j}$ is constructed from path $P_{j}^{\prime}$ as follows. Let $\left(s_{i}^{\prime}=v_{0}, v_{1}, \ldots, v_{z}=t_{i}^{\prime}\right)$ be the sequence of vertices on path $P_{j}^{\prime}$. For each vertex $v_{z^{\prime}}$ on this path, if $v_{z^{\prime}} \in K\left(u_{z^{\prime}}\right)$, then we replace $v_{z^{\prime}}$ with $u_{z^{\prime}}$. Let $P_{j}$ be the resulting path, after we erase possible cycles and consecutive occurrences of the same vertex on it. Then $P_{j}$ is a valid $s_{i}-t_{i}$ path in graph $G$, and moreover, since paths $P_{1}^{\prime}, \ldots, P_{k}^{\prime}$ were vertex disjoint, this ensures that the paths $P_{1}, \ldots, P_{k}$ are edge disjoint.

Assume now that $E^{* *}$ is any feasible solution of weight less than $\infty$ to instance $G^{\prime}$ of VC-kRC. Then all edges in $E^{* *}$ belong to set $E_{2}$. Let $E^{*}$ be the set of corresponding edges in graph $G$. Clearly, the weight of $E^{*}$ is the same as the weigh of $E^{* *}$. We only need to show that $E^{*}$ is a feasible solution to the EC-kRC instance G. Assume otherwise, and let $\left(s_{i}, t_{i}\right)$ be a source-sink pair such that $G \backslash E^{*}$ contains at least $k$ edge-disjoint paths $P_{1}, \ldots, P_{k}$ connecting $s_{i}$ to $t_{i}$. We show a collection $P_{1}^{\prime}, \ldots, P_{k}^{\prime}$ of node-disjoint paths, connecting $s_{i}^{\prime}$ to $t_{i}^{\prime}$ in $G^{\prime} \backslash E^{* *}$, thus obtaining a contradiction. Fix some $1 \leq j \leq k$. Path $P_{j}^{\prime}$ is constructed from path $P_{j}$ as follows. Let $\left(s_{i}=u_{0}, u_{1}, \ldots, u_{z}=t_{i}\right)$ be the sequence of vertices on path $P_{j}$. For each $0 \leq z^{\prime}<z$, let $e_{z^{\prime}}=\left(u_{z^{\prime}}, u_{z^{\prime}+1}\right)$. For $1 \leq z^{\prime} \leq z-1$, we replace the vertex $u_{z^{\prime}}$ on the path by two vertices: $v\left(u_{z^{\prime}}, e_{z^{\prime}-1}\right)$ and $v\left(u_{z^{\prime}}, e_{z^{\prime}}\right)$. If $s_{i}^{\prime}=v\left(s_{i}, e_{0}\right)$, then we replace $s_{i}$ with $s_{i}^{\prime}$. Otherwise, we replace it with a pair $s_{i}^{\prime}, v\left(s_{i}, e_{0}\right)$ of vertices. Similarly, if $t_{i}^{\prime}=v\left(t_{i}, e_{z-1}\right)$, then we replace $t_{i}$ with $t_{i}^{\prime}$. Otherwise, we replace it with $v\left(t_{i}, e_{z-1}\right), t_{i}^{\prime}$. Let $P_{j}^{\prime}$ denote the resulting path. It is easy to see that this is a valid path in graph $G^{\prime}$. Moreover, if paths $P_{1}, \ldots, P_{k}$ were edge disjoint, the paths $P_{1}^{\prime}, \ldots, P_{k}^{\prime}$ are guaranteed to be vertex disjoint in graph $G^{\prime}$.

\section{B VC-kRC: FROM GENERAL TO UNIFORM EDGE COSTS}

In this section, we show that in the VC-kRC problem, we can assume without loss of generality that all edges have unit weights. We will lose a $(1+1 / n)$ factor in the approximation ratio in this transformation.

Consider any VC-kRC instance $G=(V, E)$ where $w: E \rightarrow \Re^{+}$are nonnegative edge weights. Let $w_{\max }, w_{\min }$ be the maximum and minimum weights across all edges. To obtain an unweighted version, let us first consider the case when $w_{\max } / w_{\min }<n^{4}$. We can then assume without loss of generality that $w_{\min }=1$ and $w_{\max }<n^{4}$. Round the weight of every edge up to the next multiple of $1 / n^{3}$, and multiply all edge weights by $n^{3}$, obtaining integral weights $w_{e}^{\prime}$. We now replace every edge $e$ by $w^{\prime}(e)$ parallel edges. The crucial observation is that two parallel edges cannot be on different node disjoint paths, and so, in the optimal solution for the new instance, for each original edge $e \in E$, either all of its copies belong to the solution or none of them. (If only a subset of copies of $e$ belongs to the solution $E^{*}$, then deleting all copies of $e$ from $E^{*}$ still gives a feasible solution.) The cost of the optimal solution in the new instance increases by the additive factor of $1 / n$ due to the rounding of the edge weights, and since we have assumed that all edge weights are at least 1 , we lose at most a $(1+1 / n)$-factor in the solution cost.

When $w_{\max } / w_{\min }$ is not bounded by $n^{4}$, we first guess the value OPT of the optimal solution and delete all edges $e$ with $w(e)<\frac{\mathrm{OPT}}{n^{3}}$. These edges will be eventually added to our solution. Note that all such edges can contribute at most OPT $/ n$ to the solution value. For all edges of weight more than $n$. OPT, we set their new weight to be $n$. OPT, and we repeat the reduction mentioned earlier. It is easy to see that the same argument works here as well. 


\section{ACKNOWLEDGMENTS}

The first author would like to thank Sanjeev Khanna for suggesting the problem and for many interesting discussions. We would also like to thank Rajsekar Manokaran for helpful discussions on the use of the random $\kappa$-AND conjecture.

\section{REFERENCES}

Charu C. Aggarwal and James B. Orlin. 2002. On multiroute maximum flows in networks. Networks 39, 43-52. DOI : http://dx.doi.org/10.1002/net.10008

N. Alon, S. Arora, R. Manokaran, D. Moshkovitz, and O. Weinstein. 2011. Inapproximability of densest k-subgraph from average case hardness. Manuscript is available at www.csc.kth.se/ rajsekar/papers/ dks.pdf.

Y. P. Aneja, R. Chandrasekaran, S. N. Kabadi, and K. P. K. Nair. 2007. Flows over edge-disjoint mixed multipaths and applications. Discrete Applied Mathematics 155, 1979-2000. DOI:http://dx.doi.org/ 10.1016/j.dam.2007.05.001

Benny Applebaum. 2011. Pseudorandom generators with long stretch and low locality from random local one-way functions. Electronic Colloquium on Computational Complexity 18, 7.

Benny Applebaum, Boaz Barak, and Avi Wigderson. 2010. Public-key cryptography from different assumptions. In Proceedings of the 42nd ACM Symposium on Theory of Computing (STOC'10). ACM, New York, NY, 171-180.

Sanjeev Arora, James R. Lee, and Assaf Naor. 2005. Euclidean distortion and the sparsest cut. In Proceedings of the 37th ACM Symposium on Theory of Computing (STOC'05). ACM, New York, NY, 553-562. DOI : http://dx.doi.org/10.1145/1060590.1060673

Sanjeev Arora, Carsten Lund, Rajeev Motwani, Madhu Sudan, and Mario Szegedy. 1998. Proof verification and the hardness of approximation problems. Journal of the ACM 45, 3, 501-555. DOI:http://dx.doi.org/ $10.1145 / 278298.278306$

Sanjeev Arora, Satish Rao, and Umesh Vazirani. 2004. Expander flows, geometric embeddings and graph partitioning. In Proceedings of the 36th ACM Symposium on Theory of Computing (STOC'04). ACM, New York, NY, 222-231. DOI : http://dx.doi.org/10.1145/1007352.1007355

Sanjeev Arora and Shmuel Safra. 1998. Probabilistic checking of proofs: A new characterization of NP. Journal of the ACM 45, 1, 70-122. DOI : http://dx.doi.org/10.1145/273865.273901

Amitabha Bagchi, Amitabh Chaudhary, and Petr Kolman. 2003. Short length Menger's theorem and reliable optical routing. Theoretical Computer Science 339, 2-3, 246-247.

Amitabha Bagchi, Amitabh Chaudhary, Christian Scheideler, and Petr Kolman. 2007. Algorithms for faulttolerant routing in circuit-switched networks. SIAM Journal on Discrete Mathematics 21, 1, 141-157. DOI : http://dx.doi.org/10.1137/S0895480102419743

Siddharth Barman and Shuchi Chawla. 2010. Region growing for multi-route cuts. In Proceedings of the 21st Annual ACM-SIAM Symposium on Discrete Algorithms (SODA'10). 404-418. http://portal.acm.org/ citation.cfm?id=1873601.1873635

Aditya Bhaskara, Moses Charikar, Eden Chlamtac, Uriel Feige, and Aravindan Vijayaraghavan. 2010. Detecting high log-densities: An $O\left(n^{1 / 4}\right)$ approximation for densest k-subgraph. In Proceedings of the 42nd ACM Symposium on Theory of Computing (STOC'10). ACM, New York, NY, 201-210. DOI : http://dx.doi.org/10.1145/1806689.1806719

Henning Bruhn, Jakub Černý, Alexander Hall, Petr Kolman, and Jiří Sgall. 2008. Single source multiroute flows and cuts on uniform capacity networks. Theory of Computing 4, 1, 1-20. DOI:http://dx.doi.org/ 10.4086/toc.2008.v004a001

T. Chakraborty, J. Chuzhoy, and S. Khanna. 2008. Network design for vertex connectivity. In Proceedings of ACM Symposium on Theory of Computing (STOC'08). 167-176.

S. Chawla, R. Krauthgamer, R. Kumar, Y. Rabani, and D. Sivakumar. 2006. On the hardness of approximating multicut and sparsest-cut. Computational Complexity 15, 2, 94-114. DOI:http://dx.doi.org/ 10.1007/s00037-006-0210-9

Chandra Chekuri and Sanjeev Khanna. 2008. Algorithms for 2-route cut problems. In Automata, Languages and Programming. Lecture Notes in Computer Science, Vol. 5125. Springer, 472-484. http://dx.doi.org/10.1007/978-3-540-70575-8_39

E. Dahlhaus, D. S. Johnson, C. H. Papadimitriou, P. D. Seymour, and M. Yannakakis. 1994. The complexity of multiterminal cuts. SIAM Journal on Computing 23, 864-894.

Matthias Englert, Anupam Gupta, Robert Krauthgamer, Harald Räcke, Inbal Talgam, and Kunal Talwar. 2010. Vertex sparsifiers: New results from old techniques. In Approximation, Randomization, and 
Combinatorial Optimization, Algorithms and Techniques. Lecture Notes in Computer Science, Vol. 6302. Springer, 152-165. DOI : http://dx.doi.org/10.1007/978-3-642-15369-3_12

Uriel Feige. 2002. Relations between average case complexity and approximation complexity. In Proceedings of the 34th ACM Symposium on Theory of Computing (STOC'02). ACM, New York, NY, 534-543. DOI : http://dx.doi.org/10.1145/509907.509985

N. Garg, V. V. Vazirani, and M. Yannakakis. 1995. Approximate max-flow min-(multi)-cut theorems and their applications. SIAM Journal on Computing 25, 235-251.

Ralph E. Gomory and Tien Chung Hu. 1961. Multi-terminal network flows. Journal of the Society for Industrial and Applied Mathematics 9, 4, 551-570.

Ara Hayrapetyan, David Kempe, Martin Päl, and Zoya Svitkina. 2005. Unbalanced graph cuts. In Proceedings of the 13th European Symposium on Algorithms.

Subhash Khot. 2004. Ruling out ptas for graph min-bisection, densest subgraph and bipartite clique. In Proceedings of the 44th Annual IEEE Symposium on the Foundations of Computer Science (FOCS'04). $136-145$.

Subhash Khot and Nisheeth K. Vishnoi. 2005. The unique games conjecture, integrality gap for cut problems and embeddability of negative type metrics into $\ell_{1}$. In Proceedings of the 46th Annual IEEE Symposium on the Foundations of Computer Science (FOCS'05). 53-62.

W. Kishimoto. 1996. A method for obtaining the maximum multiroute flows in a network. Networks 27, $279-291$.

W. Kishimoto and M. Takeuchi. 1993. On m-route flows in a network. IEICE Transactions J-76-A, 1185-1200.

Petr Kolman and Christian Scheideler. 2011. Towards duality of multicommodity multiroute cuts and flows: Multilevel ball-growing. In Proceedings of the 28th International Symposium on Theoretical Aspects of Computer Science (STACS'11), Vol. 9. S129-140. DOI : http://dx.doi.org/10.4230/LIPIcs.STACS.2011.129

G. Kortsarz, R. Krauthgamer, and J. R. Lee. 2004. Hardness of approximation for vertex-connectivity network design problems. SIAM Journal on Computing 33, 3, 704-720.

F. T. Leighton and S. Rao. 1999. Multicommodity max-flow min-cut theorems and their use in designing approximation algorithms. Journal of the ACM 46, 787-832.

Angsheng Li and Peng Zhang. 2010. Unbalanced graph partitioning. In Algorithms and Computation. Lecture Notes on Computer Science, Vol. 6506. Springer, 218-229.

Harald Räcke. 2008. Optimal hierarchical decompositions for congestion minimization in networks. In Proceedings of the 40th ACM Symposium on Theory of Computing (STOC'08). ACM, New York, NY, $255-264$. DOI : http://dx.doi.org/10.1145/1374376.1374415

Prasad Raghavendra and David Steurer. 2010. Graph expansion and the unique games conjecture. In Proceedings of the 42nd ACM Symposium on Theory of Computing (STOC'10). ACM, New York, NY, 755-764. DOI : http://dx.doi.org/10.1145/1806689.1806792

Ran Raz. 1998. A parallel repetition theorem. SIAM Journal on Computing 27, 3, 763-803. DOI : http://dx. doi.org/10.1137/S0097539795280895

Received April 2012; revised December 2013; accepted July 2014 